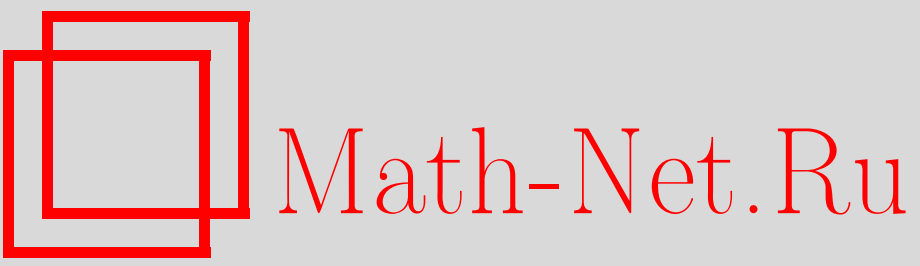

А. С. Александров, А. Д. Миронов, А. Ю. Морозов, Статистические суммы матричных моделей как первый пример специальных функций теории струн. Эрмитова одноматричная модель с матрицами конечного размера, ТMФ, 2005, том 142, номер 3, 419-488

DOI: https://doi.org/10.4213/tmf1792

Использование Общероссийского математического портала Math-Net.Ru подразумевает, что вы прочитали и согласны с пользовательским соглашением

http://www . mathnet.ru/rus/agreement

Параметры загрузки:

IP : 54.198 .64 .247

26 апреля 2023 г., $16: 13: 29$ 
ТЕОРЕТИЧЕСКАЯ

И МАТЕМАТИЧЕСКАЯ

ФИЗИКА

Том 142, № 3

март, 2005

(C) 2005 г.

А. С. Александров ${ }^{*} \dagger$, А. Д. Миронов ${ }^{*} \ddagger$,

А. Ю. Морозов*

\section{СТАТИСТИЧЕСКИЕ СУММЫ МАТРИЧНЫХ МОДЕЛЕЙ КАК ПЕРВЫЙ ПРИМЕР СПЕЦИАЛЬНЫХ ФУНКЦИЙ ТЕОРИИ СТРУН. ЭРМИТОВА ОДНОМАТРИЧНАЯ МОДЕЛЬ С МАТРИЦАМИ КОНЕЧНОГО РАЗМЕРА}

Хотя статистическими суммами матричных моделей не исчерпывается полный набор $\tau$-функций, возникающих в теории струн, они являются элементарными блоками для построения многих других $\tau$-функций и, по-видимому, правильно улавливают фундаментальную природу квантовой гравитации и теории струн. Мы предлагаем рассматривать статистические суммы матричных моделей в качестве новых специальных функций. Это означает, что они должны быть исследованы и представлены в некоторой стандартной форме безотносительно к конкретным применениям. В то же время таблицы и перечни свойств должны быть достаточно полны, для того чтобы исключить появление неожиданных особенностей в новых приложениях. Решение такой задачи требует значительных усилий, и данная статья является лишь первым шагом в этом направлении. Мы ограничимся рассмотрением одноматричной эрмитовой модели с матрицами конечного размера и сконцентрируем внимание в основном на структуре фаз и ветвей, которая возникает при рассмотрении статистической суммы как $D$-модуля. Мы обсудим роль препотенциала Качазо-Интрилигатора-Вабфы и Дийкграаффа-Вафы (который порождает некоторый базис в линейном пространстве решений условий Вирасоро, хотя понимание того, чем и как этот базис выделен, отсутствует) и вычислим несколько первых многопетлевых корреляционных функций, которые обобщают полукруговое распределение на случай полиследовых и непланарных корреляционных функций.

Ключевые слова: матричные модели, теория струн, многопетлевые корреляционные функции.

Статья написана по заказу Редколлегии.

\footnotetext{
* Институт теоретической и экспериментальной физики, Москва, Россия. E-mail: al@itep.ru, mironov@itep.ru, morozov@itep.ru

${ }^{\dagger}$ Московский физико-технический институт, Москва, Россия

‡ Физический институт им. П. Н. Лебедева РАН, Москва, Россия. E-mail: mironov@lpi.ac.ru
} 


\section{СОДЕРЖААНИЕ}

I. Теория: статистические суммы и их свойства . . . . . . . . . . . . . . . . . 421

1. Введение ....................... 421

2. Статистическая сумма как функциональный интеграл . . . . . . . . 424

2.1. Понятие статистической суммы . . . . . . . . . . . . . . . . 424

2.2. Матричная модель как простейшая струнная модель . . . . . . . . 425

3. Статистическая сумма как $D$-модуль . . . . . . . . . . . . . . . . 427

3.1. Понятие статистической суммы . . . . . . . . . . . . . . . . . . . . . . . . . . . . . . . . 427

3.2. Условия Вирасоро для ЭММКР . . . . . . . . . . . . . . . . . 429

3.3. Условия Вирасоро и петлевые операторы . . . . . . . . . . . . . . . 430

3.4. Рекуррентное решение условий Вирасоро . . . . . . . . . . . . . . . 433

4. О количестве решений . . . . . . . . . . . . . . . . . . . . . . . . . . 435

4.1. Типы зависимости от $g$. . . . . . . . . . . . . . 436

4.2. Сдвиги переменных $t$ и происхождение параметров $f:$ сверхпростой пример ...................... 437

4.3. Оператор эволюции и базис в гильбертовомпространстве решений 438

II. Конкретные специальные функции . . . . . . . . . . . . . . . . . . . . . . . 440

1. Гауссова статистическая сумма . . . . . . . . . . . . . . . . . . . . 440

2. Формулы разложения типа формулы Гивенталя для негауссовых статистических сумм КИВ-ДВ . . . . . . . . . . . . . . . . . . . . . . . . . 444

3. Препотенциал КИВ-ДВ . . . . . . . . . . . . . . . . . . . . . . . . . . . . . . . . . . . . . . . . . . . . .

III. Таблицы . . . . . . . . . . . . . . . . . . . . . . . . . . . . . . . . 454

1. Гауссова статистическая сумма . . . . . . . . . . . . . . . . . 454

1.1. Первые обобшенные числа Каталана . . . . . . . . . . . . . . . . . . 454

1.2. Первые гауссовы плотности (производяшие функции обобшенных чисел Каталана); $\nu=g N / M \ldots \ldots \ldots \ldots$. . . . . . . 455

1.3. Первые рекуррентные соотношения для гауссовых полиплотностей 456

1.4. Первые гауссовы полиплотности: аналитические выражения; $\nu=$ $g N / M ; y(z)=z^{2}-4 \nu \ldots \ldots \ldots \ldots \ldots \ldots \ldots 4$

1.5. Первые гауссовы полиплотности: первые члены $N$-разложений . 459

1.6. Рекуррентные формулы для гауссовых корреляционных функций 465

1.7. Младшие гауссовы корреляционные функции, связные и несвязные 466

1.8. Младшие препотенциалы $\mathcal{F}^{(p)}$ : первые члены разложения по $t$. . 471

1.9. Функции $Z_{G}(t \mid N)$ : первые члены разложения по $t \ldots \ldots 73$

2. Негауссовы статистические суммы . . . . . . . . . . . . 475

3. Препотенциал КИВ-ДВ: представление $\left.\mathcal{F}_{m}^{\mathrm{DV}}\right|_{t=0}$ через гауссовы препотенциалы . . . . . . . . . . . . . . . . . . . . . 476

3.1. Явные формулы для $\left.\mathcal{F}_{\mathrm{DV}}^{(p \mid m)}\right|_{t=0} \ldots \ldots \ldots \ldots \ldots . \ldots \ldots$

3.2. Пример $n=2$ (кубический препотенциал $W(z), 3 T_{3}=\kappa, M_{1}=$

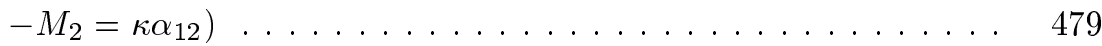

IV. Комментарии . . . . . . . . . . . . . . . . . . . . . . . 480

1. Обобшенное правило сумм (II.1.25): вывод и применение . . . . . . 480 
2. Вычисление полиплотностей в гауссовой модели . . . . . . . . . . . . . . 482

3. Непрерьвные пределы . . . . . . . . . . . . . . . . . . . . . . . . . . . . 484

\section{I. ТЕОРИЯ: СТАТИСТИЧЕСКИЕ СУММЫ И ИХ СВОЙСТВА}

\section{1. ВВЕДЕНИЕ}

Одна из целей общей теории струн [1] состоит в том, чтобы идентифицировать свойства статистических сумм различных струнных моделей. По определению статистические суммы являются производящими функциями всех корреляционных функций в квантовой теории. Существуют три различных описания/определения статистической суммы: в виде матричного элемента, в виде решения системы линейных дифференциальных уравнений (т.е. как элемента $D$-модуля) или в виде (функционального) интеграла по траекториям в конфигурационном и/или фазовом пространстве (по полевым конфигурациям), которые аналогичны трем сушествующим формулировкам квантовой механики (в виде линейной алгебры операторов в гильбертовом и фоковском пространствах, через волновые функции, через интеграл по путям). Эти совершенно различные определения подчеркивают различные свойства статистических сумм, и из их эквивалентности проистекают глубокие и нетривиальные соотношения и симметрии. Среди таких следствий важную роль играют свойства интегрируемости статистических сумм, из которых следует, что они принадлежат к классу обобщенны $\tau$-функиий [2], удовлетворяющих бесконечному набору совместных нелинейных разностно-дифференциальных уравнений (обобщенных уравнений Хироть). Более того, статистические суммы различных моделей часто бывают связаны через дуальности и/или симметрии типа зеркальной. Несмотря на общие определения и симметрии статистические суммы редко выражаются через стандартные специальные функции и обладают сложными аналитическими свойствами, со всевозможными особенностями и ветвлениями.

Все это: универсальность, богатые симметрии и невозможность свести задачу к известным функциям - означает, что статистические суммы ( $\tau$-функции) струнных моделей являются естественными кандидатами на роль следующего поколения специальных функций. Они должны быть изучены безотносительно к их конкретным применениям, их свойства должны быть исследованы и описаны, интересные частные случаи (при специальных значениях параметров, в асимптотиках, на специальных ветвях) должны быть найдены, перечислены и сведены в таблицы и в конце концов собраны в справочниках по специальным функциям. Эта задача является даже более естественной, поскольку по аналогии с обыкновенными специальными функциями (из семейств гипергеометрических, эллиптических и римановых тета-функций) все $\tau$-функции тесно связаны с теорией представлений алгебр и групп Ли [2]. Важно отметить, что, хотя статистические суммы обычно являются $\tau$-функциями, обратное неверно; статистические суммы образуют очень специальный подкласс среди $\tau$-функций - с дополнительными структурами и глубокими свойствами. (Ситуация хорошо моделируется соотношением между римановыми и общими тета-функциями: первые, представляя усеченные статистичес- 
кие суммы свободных полей на римановых поверхностях, обладают дополнительными, очень важными свойствами - такими, например, как тождества Фэя, которые следуют из теоремы Вика для свободных полей.) Другими словами, интегрируемость является естественной, но лишь малой, не исчерпывающей частью теории статистических сумм.

В данной статье мы представляем первую итерацию предложенного выше анализа/ изучения для простейшей и очень важной специальной функции теории струн - статистической суммы одноматричной әрмитовой модели с матрицами конечного размера (ЭММКР) [3]-[7]. Обшая схема рассуждений равным образом применима к любой другой матричной модели. Очевидно, что аналогичным образом следует проанализировать колчанные (конформные) матричные модели [8]-[10] (отметим также недавний прогресс в двухматричных (нормальных) моделях [11]) и $\tau$-функцию Концевича [12]-[17], после чего можно будет перейти к геометрическим $\tau$-функциям, связанным с топологическими сигма-моделями и моделями квантовой гравитации в различных фоновых полях. Однако из всех статистических сумм естественнее всего начать именно с относящихся к матричным моделям (различные аспекты матричных моделей изложены в [18]-[20]). Они не только являются простейшими примерами, но, можно ожидать, в действительности представляют элементы для построения многих других $\tau$-функций. Пример разложения геометрической статистической суммы (а именно, в случае $C P_{n}$ топологической сигма-модели) в композицию элементарных функций (в данном случае это $(n+1)$-я $\tau$-функция Концевича), линейную по всем элементам, был рассмотрен А. Гивенталем [21], [22] (см. также [10]). Более простая формула разложения для ЭММКР (которая на самом деле была получена уже в [23]) будет проанализирована в данной работе.

В стандартной теории специальных функций принято различать два уровня общности: можно рассматривать общее решение дифференциального уравнения и можно вместо этого рассмотреть частные решения (ветви), обычно связанные с характерными интегральными представлениями (и/или специальными представлениями возникающей алгебры Ли). В качестве тривиального примера можно рассмотреть цилиндрическую функцию [24] ( $D$-модуль, связанный с оператором $\left.z^{2} \partial_{z}^{2}+z \partial_{z}+\left(z^{2}-\nu^{2}\right)\right)$ и ее специальные ветви: функции Бесселя и Неймана или функции Ганкеля. Любая пара этих ветвей образует базис в пространстве решений (в $D$-модуле) и может быть зафиксирована с помошью выбора контуров интегрирования.

То же самое верно для специальных функций теории струн: специальные (функциональные) интегралы описывают специальные ветви общей статистической суммы, которая определяется набором тождеств Уорда (уравнений Швингера-Дайсона), только разница между ветвями может быть более отчетливой, чем в элементарном случае. Мы детально разберем это явление в случае ЭММКР начиная с наивного матричного интеграла в п. І.2, обобшая на произвольное решение условий Вирасоро в п. I.3 и п. I.4 (хотя сейчас мы очень мало можем сказать про случай, когда не существует разложения по родам) и возврашаясь к специальным решениям: гауссовой $\tau$-функции $Z_{\mathrm{G}}^{M}(t)$ в п. II.1 и $\tau$-функции Качазо-Интрилигатора-Вафы и Дийкграафа-Вафы (КИВ-ДВ) $Z_{\mathrm{DV}}[T \mid S](t)$ в п. II.2. С помошью формул разложсения (п. II.2), аналогичных формулам из работы Гивенталя [21], $\tau$-функция КИВ-ДВ строится из полилинейной комбинации гауссовых $\tau$-функций, и согласно аргументам п. I.4.3 такие функции можно рассматри- 
вать как базис в линейном пространстве всех решений условий Вирасоро (примерно так же, как $e^{i p x}$ в пространстве всех функций от $x$; другими словами, так же, как и $e^{i p x}$, $Z_{\mathrm{DV}}[T \mid S]$ задает ядро интегрального преобразования функций переменных $T$ к функциям переменных $S$ ). К сожалению, до сих пор не ясно, чем выделен этот базис, хотя он имеет очевидное преимушество: а именно, он задается формулами разложения, аналогичными формулам Гивенталя, и связан с теорией Уизема-Зайберга-Виттена.

В данной работе нами достигаются следуюшие четыре цели.

1. Мы формулируем задачу, состоящую в описании свойств матрично-модельных и геометрических $\tau$-функций, определенных как $D$-модули, т.е. как общие решения системы условий типа условий Вирасоро, в виде формальных рядов, и даем предварительньй вариант такого описания для ЭММКР.

2. Мы утверждаем, что описание сложной структуры фаз (ветвей) $\tau$-функции является очень важной - и до сих пор недооцененной и неисследованной - составной частью этой программы и делаем попытку такого описания для ЭММКР.

3. Мы вводим иерархическое описание фаз. Во-первых, с помощью струнной константы связи $g$ мы вводим градуировку по степеням переменных $t$ (констант связи или времен) и используем ее для того, чтобы выделить фазы, обладающие разложением по родам, для которых логарифм статистической суммы (препотенииал) допускает разложение в полубесконечный ряд по степеням $g$. Во-вторых, сдвиг конечного числа переменных $t \rightarrow t-T$ позволяет нам ввести различные виды $t$-разложений (фаз) с различными величинами (на самом деле разностями $\alpha_{i j}=\alpha_{i}-\alpha_{j}$ корней полинома степени $\left.n, W^{\prime}(z)=\sum k T_{k} z^{k-1}=\prod_{i=1}^{n}\left(z-\alpha_{i}\right)\right)$, возникающими в знаменателях. Полином $W(z)$ степени $n+1$ играет роль древесного суперпотенииала в контексте теории Дийкграафа-Вафы [25]. В третьих, каждая из этих фаз расшепляется в бесконечнопараметрическое семейство с различными положениями особенностей (точек ветвления) полиплотностей ${ }^{1)}$

$$
\rho_{W}\left(z_{1}, \ldots, z_{m}\right)=\prod_{i=1}^{m}\left(\sum_{k \geqslant 0} \frac{1}{z_{i}^{k+1}} \frac{\partial}{\partial t_{k}}\right) \mathcal{F}_{W}(t)
$$

где $Z_{W}(t)=e^{\frac{1}{g^{2}} \mathcal{F}_{W}(t)}-$ это статистическая сумма ЭММКР. Это семейство фаз параметризуется произвольной функцией $F_{W}(g)=F[g, T]=\mathcal{F}_{W}(t=0, g)$ параметра $g$ и $n$ переменных $T_{0}, \ldots, T_{n-1}$.

4. Мы утверждаем, что альтернативный подход к описанию ветвей может основываться на формулах разложения, аналогичных формулам Гивенталя, представляющих

\footnotetext{
1)Эти величины могут быть ассоциированы с многопетлевыми корреляционными функциями (корреляционными функциями вильсоновских петель) $\left\langle\left\langle\prod_{k=1}^{m} \operatorname{Tr} \frac{1}{z_{k}-\phi}\right\rangle_{W}[26]\right.$ (где двойные скобки означают связные корреляционные функции), если существует матрично-интегральное представление с некоторым пертурбативным диаграммным разложением. Мнимая часть этих корреляционных функций естественным образом связана с полиплотностями распределения собственных значений в данном случае. Именно поэтому мы обычно называем $\rho_{W}\left(z_{1}, \ldots, z_{m}\right)$ полиплотностями. Также мы будем называть их полирезольвентами, поскольку $\rho(z)$ - это резольвента.
} 
матрично-модельные/геометрические $\tau$-функции с помошью оператора, действуюшего на произведение “элементарных $\tau$-функций”. В случае ЭММКР “элементарными $\tau$-функциями” являются гауссовы, и формулы разложения приводят к $\tau$-функциям КИВ-ДВ $Z_{\mathrm{DV}}[T \mid S](t)$. Если задан полином $W(z)$, то обшая статистическая сумма, построенная по произвольной функции $Z[T]=e^{F[T] / g^{2}}$ с помошью оператора эволюции $\check{U}_{W}(t)$ (см. п. I.4.3),

$$
Z_{W}(t)=\breve{U}_{W}(t) Z[T]
$$

может быть представленна через $\tau$-функции КИВ-ДВ

$$
Z_{W}(t)=\int Z_{\mathrm{DV}}[T \mid S](t) C[S] d S
$$

где коэффициенты $C[S]$ определяются разложением “начальной” функции $Z[T]$ в линейную комбинацию $Z_{\mathrm{DV}}[T \mid S]=e^{F_{\mathrm{DV}}[T \mid S] / g^{2}}=Z_{\mathrm{DV}}[T \mid S](t=0)$,

$$
Z[T]=\int Z_{\mathrm{DV}}[T \mid S] C[S] d S .
$$

\section{2. СТАТИСТИЧЕСКАЯ СУММА КАК ФУНКЦИОНАЛЬНЫЙ ИНТЕГРАЛ}

2.1. Понятие статистической суммы. Теория струн изучает статистические суммы

$$
\begin{gathered}
Z_{\mathcal{M}, S_{0}}\left\{t \mid \phi_{0}\right\}=\int_{\mathcal{M}} d \phi \exp \left(\frac{S\left(\phi_{0}+\phi\right)}{g}\right), \\
S(\phi)=S_{0}(\phi)+\sum_{\vec{k}} t_{\vec{k}} V_{\vec{k}}(\phi)
\end{gathered}
$$

(с полным набором вертексных операторов $\left.V_{\vec{k}}(\phi)\right)$ как функции:

а) пространства полей $\mathcal{M}$, параметризованного $\phi$ и снабженного некоторой "естественной" мерой $d \phi$,

б) меры $d \mu(\phi)=d \phi e^{\frac{1}{g} S_{0}(\phi)}$ на $\mathcal{M}\left(\right.$ составленной из $d \phi$, затравочного действия $S_{0}(\phi)$ и струнной константы связи/постоянной Планка $g$ ),

в) бесконечного множества констант связи $t_{\vec{k}}$ и

г) фонового состояния (вакуума) $\phi_{0}$.

Обычно фоновое поле $\phi_{0}$ является решением уравнений движения

$$
\left.\frac{\delta S_{0}(\phi)}{\delta \phi}\right|_{\phi_{0}}=0
$$

но иногда используют их квантовый аналог

$$
\left.\frac{\delta d \mu(\phi)}{\delta \phi}\right|_{\phi_{0}}=0
$$

и/или деформированное действие $S(\phi)$, подставленное вместо $S_{0}(\phi)$ в (I.2.2) и (I.2.3) (таким образом, зависимости от $\phi_{0}, g$ и $t_{\vec{k}}$ смешиваются). 
Пространство $\mathcal{M}$ вместе с возможными ограничениями на выбор меры $d \mu(\phi)$ определяет конкретную струнную модель.

В задачи теории струн входит также поиск универсального метода построения всех интересных пространств $\mathcal{M}$ из некоторых строительных блоков (например, из элементарных симплексов).

Обычно

$$
\mathcal{M}=\operatorname{Hom}(W, T) / \mathcal{G}
$$

является пространством отображений мировой поверхности $W$ в таргет-пространство $T$, факторизованное по некоторой группе симметрий $\mathcal{G}$.

Для любого $\mathcal{M}$ можно построить новые пространства $\mathcal{M}_{G} \equiv \mathcal{M} \otimes G_{R} / U$, где $G_{R}$ это группа $G$ в некотором представлении $R$, факторизованная по действию своей максимальной подгрупшы $U$.

Характерным примером является $G_{R}=M_{N}$, пространство квадратных $N \times N$ матриц ( $N$-мерное представление $G L(N)$ - пространства линейных отображений векторного пространства $V_{N}$ в себя), на котором унитарная группа $U(N)$ действует сопряжением $M \rightarrow U^{-1} M U$. Этот рецепт "бранизации” (замены чисел матрицами) известен из изучения "стопок бран" [27].

Для пространства $\mathcal{M}$ типа (I.2.4) можно перейти к отображениям между более общими расслоениями, пучками и комплексами над $W$ и $T$ (заменяя числа отображсениями комплексов, с комплексами, описываемыми вспомогательными переменными, как, например, в формализмах Баталина-Фрадкина и Баталина-Фрадкина-Вилковыского $[28]-[33])$.

Для заданного вакуума $\phi_{0}$ часто имеет смысл переопределить набор констант связи в действии $S(\phi)$ в формуле (I.2.1). Это всегда возможно, если вертексные операторы $V_{\vec{k}}(\phi)$ образуют полныи й набор (в сильном или слабом смысле, подробности см. в [34]):

$$
\begin{aligned}
S\left(\phi_{0}+\phi\right) & =S_{0}\left(\phi_{0}+\phi\right)+\sum_{\vec{k}} t_{\vec{k}} V_{\vec{k}}\left(\phi_{0}+\phi\right)= \\
& =S_{0}\left(\phi_{0}+\phi\right)+\sum_{\vec{k}} t_{\vec{k}}^{\left\{\phi_{0}\right\}} V_{\vec{k}}(\phi) .
\end{aligned}
$$

Константы связи $t_{k}^{\left\{\phi_{0}\right\}}$ лучше, чем $t_{k}$, подходят для построения теории возмушений вокруг конкретного фонового значения $\phi_{0}$, но они сами зависят от $\phi_{0}$, и тождества Уорда (такие как условия Вирасоро, представленные ниже), выраженные через них, имеют более сложную форму. Конечно, все $t_{k}^{\left\{\phi_{0}\right\}}=0$, если $t_{k}=0$.

2.2. Матричная модель как простейшая струнная модель. Самый первый пример струнной модели $-W=p t, T=p t$ (т.е. как мировая поверхность, так и таргет-пространство являются точками). Простейшая связанная с такой моделью "стопка бран" имеет вид $\mathcal{M}=M_{N} / U(N)$, и статистическая сумма соответствует әрмитовой одноматричной модели картановского типа [6]:

$$
\mathcal{Z}_{N}^{(\text {matr })}(t)=\frac{1}{\operatorname{Vol}_{U(N)}} \int_{M_{N}} D \phi \exp \left(\frac{1}{g} \sum_{\vec{k}} t_{\vec{k}} \operatorname{Tr} \phi^{k_{1}} \cdot \ldots \cdot \operatorname{Tr} \phi^{k_{m}}\right),
$$


где суммирование проводится по всем неотрицательным целозначным векторам $\vec{k}=$ $\left(k_{1}, \ldots, k_{m}\right)$ со всеми возможными длинами $m$. Мера

$$
D \phi=\prod_{i, j=1}^{N} d \phi_{i j}
$$

является $U(N)$-инвариантной, и если $\phi=U^{-1} \phi_{D} U$, где $\phi_{D}=\operatorname{diag}\left(\phi_{i}\right)$, то

$$
D \phi=D U \prod_{i=1}^{N} d \phi_{i} \prod_{i<j}^{N}\left(\phi_{i}-\phi_{j}\right)^{2},
$$

$D U$ - это мера Хаара на группе $U_{N}$, индуцированная нормой $\|\delta U\|^{2}=\operatorname{Tr}\left(U^{-1} \delta U\right)^{2}$, а объем унитарной группы

$$
\mathrm{Vol}_{U(N)}=\int_{U(N)} D U \sim \prod_{k=1}^{N-1} k ! .
$$

Эта модель - картановского типа, поскольку интеграл сводится к $N$-кратному интегралу только по собственным значениям $\phi_{i}$. Контуры интегрирования не обязательно должны быть действительными прямыми (такие интегралы можно рассматривать в качестве аналитического продолжения, см. примеры в конце п. І.3.1).

Обычно вместо $\mathcal{Z}_{N}(t)$ рассматривается усеченная статистическая сумма $Z_{N}(t)$ с ненулевыми временами $t_{\vec{k}}$, соответствуюшими только векторам $\vec{k}$ единичной длины,

$$
Z_{N}^{(\mathrm{matr})}(t)=\frac{1}{\operatorname{Vol}_{U(N)}} \int_{M_{N}} D \phi \exp \left(\frac{1}{g} \sum_{k \in Z_{+}} t_{k} \operatorname{Tr} \phi^{k}\right) .
$$

Именно этот объект мы будем использовать в качестве прототипа статистической суммы ЭММКР (с конечным $N$ ).

Существует целый набор мощных методов, предназначенных для исследования усеченной модели (I.2.10), рассматриваемой как модель картановского типа [6], [7]. Двумя наиболее важными из них являются метод ортогональных полиномов [3] и формализм свободных фермионов [5], [15]. Используя их, можно вывести детерминантное представление [5]-[7] функции $Z_{N}$

$$
Z_{N}^{(\text {matr })}(t)=\operatorname{det}_{0 \leqslant i, j<N} \mathcal{H}_{i+j}(t)
$$

где

$$
\mathcal{H}_{j}=\int \phi^{j} \exp \left(\frac{1}{g} \sum_{k=0}^{\infty} t_{k} \phi^{k}\right) d \phi .
$$

В свою очередь, это представление можно использовать для доказательства [4] того, что $Z_{N}$ является $\tau$-функцией для иерархии цепочки Тоды, т.е. удовлетворяет бесконечной системе билинейных дифференциальных уравнений. Например,

$$
Z_{N}^{(\text {matr })} \frac{\partial^{2} Z_{N}^{(\text {matr })}}{\partial t_{1}^{2}}-\frac{\partial Z_{N}^{(\text {matr })}}{\partial t_{1}} \frac{\partial Z_{N}^{(\text {matr })}}{\partial t_{1}}=Z_{N-1}^{(\text {matr })} Z_{N+1}^{(\text {matr })} .
$$


В формуле (I.2.10) затравочное действие отсутствует. Как в каждой струнной модели, его можно ввести с помощью сдвига констант связи $t_{k} \rightarrow t_{k}-T_{k}$ :

$$
Z_{N, W}^{(\mathrm{matr})}(t)=\frac{1}{\operatorname{Vol}_{U(N)}} \int d \phi \exp \left(-\frac{1}{g} \operatorname{Tr} W(\phi)\right) \exp \left(\frac{1}{g} \sum_{k} t_{k} \operatorname{Tr} \phi^{k}\right),
$$

где $W(z)=\sum_{k=1}^{n+1} T_{k} z^{k}$. Несмотря на то что формула (І.2.14) получена с помошью простого сдвига переменных, эта статистическая сумма сушественно зависит от потенциала $W(z)$, особенно от его степени $n+1$, поскольку изменение $n$ полностью меняет структуру ряда теории возмушений. Другими словами, система претерпевает фазовый переход (статистическая сумма сингулярна) при нулевом значении коэффициента перед лидируюшим $\left(z^{n+1}\right)$ членом $\left.{ }^{2}\right) W(z)$.

\section{3. СТАТИСТИЧЕСКАЯ СУММА КАК $D$-МОДУЛЬ}

3.1. Понятие статистической суммы. Статистическая сумма ( $\tau$-функция) является в действительности очень сложным объектом. На самом деле это даже не функция, а формальный $D$-модуль, т.е. полный набор степенных рядов (по переменным $t$ ), удовлетворяющих непротиворечивой системе линейных уравнений. Решение системы не обязательно должно быть единственным, однако подходящее аналитическое продолжение по переменным $t$ переводит одно решение в другое, и на достаточно большом пространстве модулей (констант связи $t$ ) можно рассматривать объект как целое, по крайней мере формально, и именно его мы будем называть статистической суммой. Наивно различные решения можно интерпретировать как различные ветви статистической суммы, связанные с различными фазами теории. Далее, решения линейных дифференциальных уравнений часто можно представить в виде интегралов (по спектральнылм многообразиям), но "контуры" интегрирования остаются произвольными: это могут быть произвольные цепи с комплексными коэффициентами (для целых коэффициентов этот произвол обычно описывают в терминах монодромий, но в случае статистических сумм коэффициенты не обязательно должны быть целыми). Модель статистической суммы - это интегральная формула, в которой содержится достаточно много свободных параметров для того, чтобы представить обшее решение рассматриваемых дифференциальных уравнений.

Чтобы прояснить нашу точку зрения, рассмотрим ряд примеров.

Во-первых, nлоские $S U(2)$-модули $a(\lambda)$ теории Зайберга-Виттена [35] удовлетворяют уравнению

$$
\left(4 \lambda(\lambda-1) \partial_{\lambda}^{2}+1\right) a(\lambda)=0,
$$

которое имеет интегральное представление

$$
a(\lambda)=\int_{C} \sqrt{\frac{x-\lambda}{x(x-1)}} d x
$$

\footnotetext{
2)Эта ситуация очень похожа на ситуацию в матричных моделях Концевича, которые кардинальным образом зависят от матрично-модельного потенциала, особенно от его степени [16].
} 
а модель задается произвольным контуром

$$
C=\alpha[0,1]+\beta[1, \lambda]
$$

которьй является произвольной линейной комбинацией (с комплексными(!) коэффициентами) циклов $A$ и $B$ на торической спектральной кривой. Существует альтернативное описание того же объекта (этой “игрушечной” статистической суммы) в терминах 1-формы Зайберга-Виттена

$$
d S(x, \lambda)=\sqrt{\frac{x-\lambda}{x(x-1)}} d x
$$

связанной с исходным уравнением (I.3.1) через соотношение

$$
\left(4 \lambda(\lambda-1) \partial_{\lambda}^{2}+1\right) d S(x, \lambda)=d \sqrt{\frac{x(x-1)}{x-\lambda}} \cong 0
$$

(где $\cong$ означает эквивалентность в когомологиях).

Другим примером, уже обсуждавшимся во введении, являются цилиндрические функции. Они определяются уравнением

$$
\left[\lambda^{2} \partial_{\lambda}^{2}+\lambda \partial_{\lambda}+\left(\lambda^{2}-\nu^{2}\right)\right] Z_{\nu}(\lambda)=0
$$

а их интегральное представление имеет вид

$$
Z_{\nu}(x)=\frac{1}{2 \pi} \int_{C} \exp (-i x \sin \theta+i \nu \theta) d \theta
$$

Модель задается произвольной линейной комбинацией двух контуров, например таких, как контуры, выбранные в формуле (8.423) в [24] (этот выбор фиксирует функции Ганкеля в качестве базиса).

Еще одним примером является функция Эйри (простой пример модели Концевича [12]-[17]), заданная определяюшим уравнением

$$
\left(\partial_{\lambda}^{2}+\lambda\right) A(\lambda)=0
$$

с интегральным представлением

$$
A(\lambda)=\int_{C} \exp \left(\frac{x^{3}}{3}+\lambda x\right) d x .
$$

Этот $D$-модуль является частным случаем цилиндрической функции с $\nu=1 / 3$. 
3.2. Условия Вирасоро для ЭММКР. Возврашаясь к ЭММКР, отметим, что она исходно была определена в терминах матричного интеграла (I.2.10). Этот интеграл зависит от (бесконечного множества) параметров $t_{k}$ и, согласно приведенным выше аргументам, подразумевает другое определение статистической суммы - как решения (бесконечной) системы согласованных линейных (дифференциальных) уравнений (тождеств Уорда или уравнений Пикара-Фукса), т.е. как $D$-модуль. В конкретном случае ЭММКР эти уравнения ${ }^{3)}$,

$$
\begin{gathered}
\hat{L}_{m} Z(t)=0, \quad m \geqslant-1, \\
\hat{L}_{m}=\sum_{k \geqslant 0} k t_{k} \frac{\partial}{\partial t_{k+m}}+g^{2} \sum_{\substack{a+b=m \\
a, b \geqslant 0}} \frac{\partial^{2}}{\partial t_{a} \partial t_{b}},
\end{gathered}
$$

называются [37] условиями Вирасоро, поскольку $\left[\hat{L}_{m}, \hat{L}_{n}\right]=(m-n) \hat{L}_{m+n}$ (на самом деле это только борелевская подалгебра алгебры Вирасоро).

Впредь мы будем определять статистическую сумму ЭММКР как обшее решение системы (I.3.10), так что матричный интеграл (І.2.10) является частным интегральным представлением. Одной из наших задач будет проанализировать, насколько обшим является это интегральное представление и можно ли считать его моделью статистической суммы ЭММКР.

Поскольку

$$
\left[\frac{\partial}{\partial t_{0}}, \hat{L}_{m}\right]=0
$$

то можно диагонализовать оператор $\partial / \partial t_{0}$ и наложить следующие дополнительные условия на статистическую сумму:

$$
\frac{\partial}{\partial t_{0}} Z_{S}=S Z_{S}
$$

с постоянными $S$. Интегральные представления (I.2.10) и (I.2.14) с очевидностью удовлетворяют этому соотношению с целым $S=N$. Обшее решение условий Вирасоро можно разложить в линейную комбинацию функций $Z_{S}$ с различными постоянныци $S$, но в действительности такое разложение выглядит не слишком естественно ${ }^{4)}$.

\footnotetext{
3) Эти уравнения выводятся из инвариантности интеграла (І.2.10) относительно замены переменной интегрирования $\phi \rightarrow \phi+\epsilon_{m} \phi^{m+1}$ [36]. Аналогично интеграл (I.2.6) инвариантен относительно замен $\phi \rightarrow \phi+\epsilon_{m, \vec{k}} \phi^{m+1} \operatorname{Tr} \phi^{k_{1}} \cdot \ldots \cdot \operatorname{Tr} \phi^{k_{l}}$. Получающийся набор условий является следствием уравнений (Т.3.10) и очевидных соотношений

$$
\frac{\partial \mathcal{Z}}{\partial t_{\vec{k}}}=\frac{\partial^{m} \mathcal{Z}}{\partial t_{k_{1}} \ldots \partial t_{k_{m}}} .
$$

Обратный путь от уравнений (Т.3.10) к интегральному представлению (I.2.10) обсуждается в работах [8].

4) Еще хуже то, что, хотя условия $N_{i}=$ const в формулах разложения типа формулы Гивенталя (см. п. II.2) являются очевидными обобщениями условия (І.3.13) (и, более того, $\sum_{i=1}^{n} N_{i}=N=$ $S)$, операторы, собственными числами которых являлись бы $N_{i}$, неизвестны. Это вызывает проблемы с обоснованием линейного разложения каждой ветви статистической суммы по функциям Гивенталя (см. далее формулу (II.2.4)) с постоянными $N_{i}$.
} 
Отметим, что при конкретных значениях $N$ статистическая сумма $Z_{N}^{(\text {matr) }}$ удовлетворяет дополнительным дифференциальным уравнениям, которые следуют из того факта, что $\operatorname{Tr} \phi^{k}$ при $k>N$ можно выразить через алгебраическую комбинацию $\operatorname{Tr} \phi^{k} \mathrm{c} k \leqslant N$. Например, при $N=1$

$$
\left(\frac{\partial}{\partial t_{k}}-\left(\frac{\partial}{\partial t_{1}}\right)^{k}\right) Z_{N=1}^{(\text {matr })}=0 .
$$

Мы не накладываем эти ограничения в нашем определении обшей статистической суммы ЭММКР - единственными условиями являются (I.3.10) (и (I.3.16), которые определены ниже).

Можно искать решения системы (І.3.10) в классе формальных рядов по степеням $t_{k}$, и эти ряды будут различаться теми комбинациями $t_{k}$, которые могут находиться в знаменателях. Различные такие возможности можно проинтерпретировать как различные $\oint a з ы$ матричной модели или как различные ветви ее статистической суммы - $\tau$-функции $Z(t)$. Формально их можно рассматривать как различные приближения и аналитические продолжения интеграла (I.2.10). Эти различные фазы параметризуются, в частности, различными затравочными действиями, т.е. рассматривается интеграл (I.2.14), а не (I.2.10).

Введение затравочного действия $W(\phi)=\sum_{k=1}^{n+1} T_{k} \phi^{k}$ изменяет условия Вирасоро (I.3.10) тривиальным образом, при помощи сдвига

$$
\hat{L}_{m} \rightarrow \hat{L}_{m}^{W}=\hat{L}_{m}-\sum_{k \geqslant 0} k T_{k} \frac{\partial}{\partial t_{k+m}}
$$

но решения $Z_{W}(t)=e^{\mathcal{F}_{W}(t) / g^{2}}$ теперь можно искать среди формальных рядов по положительным степеням переменных $t$ с $T$, возникающими в знаменателях коэффициентов этих рядов. Теперь к тому же набор условий Вирасоро надо дополнить условиями

$$
\frac{\partial Z_{W}}{\partial T_{k}}+\frac{\partial Z_{W}}{\partial t_{k}}=0 \quad \forall k=0, \ldots, n+1
$$

3.3. Условия Вирасоро и петлевые операторы. При работе с условиями Вирасоро (І.3.10) очень полезными оказываются петлевой оператор

$$
\widehat{\nabla}(z)=\sum_{k \geqslant 0} \frac{1}{z^{k+1}} \frac{\partial}{\partial t_{k}}
$$

и два дополняющих друг друга проектора $\widehat{P}_{z}^{\mp}$, проецирующие на отрицательные и неотрицательные степени $z$,

$$
\begin{aligned}
& \widehat{P}_{z}^{-}\left[\sum_{k=-\infty}^{+\infty} \xi_{k} z^{k}\right]=\sum_{k<0} \xi_{k} z^{k}, \\
& \widehat{P}_{z}^{+}\left[\sum_{k=-\infty}^{+\infty} \xi_{k} z^{k}\right]=\sum_{k \geqslant 0} \xi_{k} z^{k} .
\end{aligned}
$$


С помошью этих операторов и производяшей функции

$$
v(z)=\sum_{k \geqslant 0}^{\infty} t_{k} z^{k}
$$

можно переписать полньй набор условий Вирасоро (Т.3.10) в виде одного петлевого уравнения (см. [26])

$$
\widehat{P}_{z}^{-}\left[v^{\prime}(z) \rho(z)\right]+\rho^{2}(z)+g^{2} \widehat{\nabla}(z) \rho(z)=0
$$

на резольвенту $(\text { плотность })^{5)}$

$$
\rho(z) \equiv \widehat{\nabla}(z) \mathcal{F} \equiv \sum_{k \geqslant 0} \frac{1}{z^{k+1}} \frac{\partial \mathcal{F}}{\partial t_{k}}, \quad \mathcal{F}=g^{2} \ln Z
$$

Уравнение (І.3.20) необходимо дополнить условием нулевой кривизны

$$
\widehat{\nabla}(x) \rho(z)=\widehat{\nabla}(z) \rho(x) .
$$

После сдвига (Т.3.15) модифицированные условия Вирасоро для функции

$$
\rho_{W}(z)=\widehat{\nabla}(z) \mathcal{F}_{W}
$$

имеют вид

$$
\widehat{P}_{z}^{-}\left[W^{\prime}(z) \rho_{W}(z)\right]=\rho_{W}^{2}(z)+g^{2} \widehat{\nabla}(z) \rho_{W}(z)+\widehat{P}_{z}^{-}\left[v^{\prime}(z) \rho_{W}(z)\right] .
$$

В нижеследуюшем рассмотрении мы будем использовать еше один петлевой оператор, определенный для произвольной функции $u(z)$,

$$
\widehat{R}_{u}(z)=\sum_{l \geqslant 0} \widehat{P}_{z}^{+}\left[\frac{u^{\prime}(z)}{z^{l+1}}\right] \frac{\partial}{\partial t_{l}} .
$$

5) Условия Вирасоро (І.3.10) можно построить в терминах свободных полей. В самом деле, рассмотрим $U(1)$-ток $\partial_{z} \phi(z)$ в теории одного свободного скалярного поля, реализованного алгеброй Гейзенберга, действующей на пространстве функций времен $t_{k}$,

$$
\partial_{z} \phi(z)=\frac{1}{g} v^{\prime}(z)+g \widehat{\nabla}(z) .
$$

Тогда генераторы алгебры Вирасоро задаются стандартным разложением тензора энергии-импульса

$$
T(z) \equiv \frac{1}{2}:\left(\partial_{z} \phi(z)\right)^{2}:=\sum \frac{L_{n}}{z^{n+2}} .
$$

Теперь петлевое уравнение является просто требованием того, что статистическая сумма как функция времен задает вакуум по отношению к алгебре Вирасоро:

$$
\widehat{P}_{z}^{-} T(z) Z=0 .
$$

Этот язык оказался очень эффективным при работе с различными колчанными моделями и с непрерывными пределами (см. [38], [8]). 
Характерным свойством этого оператора является то, что для произвольной функции $g(t)$ времен выполняется следующее равенство:

$$
\widehat{P}_{z}^{+}\left[u^{\prime}(z) \widehat{\nabla}(z) g(t)\right]=\widehat{R}_{u}(z) g(t) .
$$

В частности, если $u(z)$ - это потенциал $v(z)$ или $W(z)$ и $g(t)-$ это $\mathcal{F}(t)=g^{2} \ln Z$ или $\mathcal{F}_{W}(t)=\mathcal{F}(T, t)=g^{2} \ln Z_{W}$, то для соответствуюших членов в петлевых уравнениях (I.3.20) и (І.3.24) получаются соотношения

$$
\widehat{P}_{z}^{-}\left[v^{\prime}(z) \rho(z)\right]=v^{\prime}(z) \rho(z)-\widehat{P}_{z}^{+}\left[v^{\prime}(z) \rho(z)\right]=v^{\prime}(z) \rho(z)-\widehat{R}_{v}(z) \mathcal{F}
$$

И

$$
\widehat{P}_{z}^{-}\left[W^{\prime}(z) \rho_{W}(z)\right]=W^{\prime}(z) \rho_{W}(z)-\widehat{P}_{z}^{+}\left[W^{\prime}(z) \rho_{W}(z)\right]=W^{\prime}(z) \rho_{W}(z)-\widehat{R}_{W}(z) \mathcal{F}_{W}
$$

Для полиномиального потенциала $W(z)$ последний член в правой части равенств является полиномом степени $n-1$ по $z$. Более того, как следствие (І.3.16) получается соотношение

$$
\check{R}_{W}(z) \mathcal{F}_{W}=-\sum_{k, l \geqslant 0}(k+l+2) T_{k+l+2} z^{k} \frac{\partial \mathcal{F}_{W}}{\partial T_{l}} .
$$

В дальнейшем мы будем использовать значок - для обозначения операторов, содержаших производные по $T$, а операторы, содержашие производные по $t$, помечаются знач-

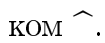

С помошю $\widehat{R}_{W}(z)$ можно переписать (І.3.24) в наиболее удобной форме:

$$
W^{\prime}(z) \rho_{W}(z)=\rho_{W}^{2}(z)+f_{W}(z)+g^{2} \widehat{\nabla}(z) \rho_{W}(z)+\widehat{P}_{z}^{-}\left[v^{\prime}(z) \rho_{W}(z)\right],
$$

где

$$
f_{W}(z)=\widehat{P}_{z}^{+}\left[W^{\prime}(z) \rho_{W}(z)\right]=\widehat{R}_{W} \ln \mathcal{F}_{W} .
$$

Отметим также, что

$$
\widehat{\nabla}(x) v(z)=\widehat{P}_{x}^{-}\left[\frac{1}{z-x}\right], \quad \widehat{\nabla}(x) v^{\prime}(z)=-\widehat{P}_{x}^{-}\left[\frac{1}{(z-x)^{2}}\right],
$$

и для любой функции $h(z)$ такой, что $\widehat{P}_{z}^{+}[h(z)]=0$, имеем

$$
\widehat{\nabla}(x) P_{z}^{-}\left[v^{\prime}(z) h(z)\right]=-P_{z}^{-}\left[P_{x}^{-}\left[\frac{h(z)}{(z-x)^{2}}\right]\right]=\partial_{x} \frac{h(z)-h(x)}{z-x},
$$

где $\partial_{x}=\partial / \partial x$. Справедлива также следуюшая формула для коммутатора:

$$
\left[\widehat{\nabla}(x), \widehat{R}_{v}(z)\right]=\partial_{x} \widehat{P}_{z}^{+}\left[\frac{\widehat{\nabla}(z)}{z-x}\right] .
$$


3.4. Рекуррентное решение условий Вирасоро. В этом пункте мы будем считать, что $Z_{W}(t)$ обладает разложением по родам, т.е. что

$$
\ln Z_{W}(t)=g^{-2} \mathcal{F}_{W}(t)=\sum_{p \geqslant 0} g^{2 p-2} \mathcal{F}_{W}^{(p)}(t)
$$

(это предположение будет обсуждаться в п. І.4.1). Статистическая сумма может быть получена одной из следующих процедур:

методом условий Вирасоро, т.е. решением петлевых уравнений (I.3.30), и

методом Гивенталя, т.е. с помошью формулы разложения (II.2.4), которая будет введена ниже в п. II.2.

В рамках метода Гивенталя произвольная фаза ЭММКР с заданным потенциалом $W(\phi)$ степени $n+1$ описывается согласно формуле разложения (II.2.4) в терминах $n$ гауссовых моделей. Это означает, что средние зависят от дополнительного набора переменных $N_{i}$, характеризуюших конкретную седловую точку (вакуум) ЭММКР. Мы вернемся к этому вопросу более подробно в п. II.2, а здесь рассмотрим условия Вирасоро (І.3.30), которые априори задают более богатое множество возможных решений/фаз (хотя все они должны быть линейными комбинациями решений в форме Гивенталя).

Чтобы преобразовать уравнение (І.3.30) в решаемую систему рекуррентных соотношений, разложим

$$
\rho_{W}(z \mid t)=g^{2} \widehat{\nabla}(z) \ln Z_{W}(t)=\widehat{\nabla}(z) \mathcal{F}_{W}(t)
$$

в ряд по степеням $g^{2}$ и $t$ :

$$
\begin{aligned}
\rho_{W}(z \mid t) & =\sum_{p, m \geqslant 0} \frac{g^{2 p}}{m !} \oint \cdots \oint v\left(z_{1}\right) \ldots v\left(z_{m}\right) \rho_{W}^{(p \mid m+1)}\left(z, z_{1}, \ldots, z_{m}\right), \\
f_{W}(z \mid t) & =\sum_{p, m \geqslant 0} \frac{g^{2 p}}{m !} \oint \cdots \oint v\left(z_{1}\right) \ldots v\left(z_{m}\right) f_{W}^{(p \mid m+1)}\left(z \mid z_{1}, \ldots, z_{m}\right) .
\end{aligned}
$$

Таким образом мы вводим полный набор полиплотностей

$$
\rho_{W}^{(p \mid m)}\left(z_{1}, \ldots, z_{m}\right)=\left.\hat{\nabla}\left(z_{1}\right) \ldots \hat{\nabla}\left(z_{m}\right) \mathcal{F}_{W}^{(p)}\right|_{t=0}
$$

и вспомогательных полиномов (которые задают отличие между различными фазами при заданном $W(z))$

$$
f_{W}^{(p \mid m+1)}\left(z \mid z_{1}, \ldots, z_{m}\right)=\check{R}_{W}(z) \rho_{W}^{(p \mid m)}\left(z_{1}, \ldots, z_{m}\right) .
$$

Выполнение соотношения (I.3.39) гарантирует, что условия (I.3.16) также выполняютcя. 
Действуя на уравнение (І.3.30) оператором $\widehat{\nabla}\left(z_{1}\right) \ldots \widehat{\nabla}\left(z_{m}\right)$, используя соотношение (I.3.33) и положив потом все $t_{k}=0$, мы получим рекуррентное (как по $p$, так и по $m$ ) выражение, определяющее полиплотности $\rho_{W}^{(p \mid m)}$ :

$$
\begin{aligned}
& W^{\prime}(z) \rho_{W}^{(p \mid m+1)}\left(z, z_{1}, \ldots, z_{m}\right)-f_{W}^{(p \mid m+1)}\left(z \mid z_{1}, \ldots, z_{m}\right)= \\
& =\sum_{q} \sum_{m_{1}+m_{2}=m} \rho_{W}^{\left(q \mid m_{1}+1\right)}\left(z, z_{i_{1}}, \ldots, z_{i_{m_{1}}}\right) \rho_{W}^{\left(p-q \mid m_{2}+1\right)}\left(z, z_{j_{1}}, \ldots, z_{j_{m_{2}}}\right)+ \\
& \quad+\sum_{i=1}^{m} \frac{\partial}{\partial z_{i}} \frac{\rho_{W}^{(p \mid m)}\left(z, z_{1}, \ldots, \check{z}_{i}, \ldots, z_{m}\right)-\rho_{W}^{(p \mid m)}\left(z_{1}, \ldots, z_{m}\right)}{z-z_{i}}+ \\
& \quad+\widehat{\nabla}(z) \rho_{W}^{(p-1 \mid m+1)}\left(z, z_{1}, \ldots, z_{m}\right) .
\end{aligned}
$$

Совместно с (I.3.39) этого соотношения достаточно для нахождения явных выражений для всех полиплотностей через $W(z)$ и полиномы $f_{W}^{(p \mid 1)}(z)$. В действительности последние (полиномы $f_{W}^{(p \mid 1)}(z)$, все имеюшие степень $n-1$ ) не являются независимыми, поскольку (І.3.39) для $m=0$,

$$
f_{W}^{(p \mid 1)}(z)=\check{R}_{W}(z) F^{(p)}[T]
$$

выполняется благодаря условию нулевой кривизны (І.3.22) и выражает все полиномы $f$ через единственную функцию параметров $T$ (т.е. функцию $W(z))$ и $g$, т.е. через препотенциал при $t=0$ :

$$
\mathcal{F}_{W}(t=0, g)=F[g, T]=\sum_{p=0}^{\infty} g^{2 p} F^{(p)}[T] .
$$

Зависимость функции $F[g, T]$ от $T_{n}$ и $T_{n+1}$ не произвольна, поскольку условия Виpacopo $\hat{L}_{-1}^{W}$ и $\hat{L}_{0}^{W}$ можно корректным образом ограничить на $t=0$ и затем выразить $\partial F / \partial T_{n+1}$ и $\partial F / \partial T_{n}$ через $\partial F / \partial T_{l}$ с $l=0, \ldots, n-1$ :

$$
\begin{aligned}
\frac{\partial F^{(0)}}{\partial T_{n}}= & -\frac{1}{(n+1) T_{n+1}} \sum_{l=0}^{n-1}(l+1) T_{l+1} \frac{\partial F^{(0)}}{\partial T_{l}} \\
\frac{\partial F^{(0)}}{\partial T_{n+1}}= & \frac{1}{(n+1) T_{n+1}}\left(\frac{\partial F^{(0)}}{\partial T_{0}}\right)^{2}-\frac{1}{(n+1) T_{n+1}} \sum_{l=0}^{n-1} l T_{l} \frac{\partial F^{(0)}}{\partial T_{l}}- \\
& -\frac{n T_{n}}{(n+1)^{2} T_{n+1}^{2}} \sum_{l=0}^{n-1}(l+1) T_{l+1} \frac{\partial F^{(0)}}{\partial T_{l}}
\end{aligned}
$$

Из формулы (I.3.40) при $p=0$ и $m=0$ немедленно следует, что

$$
\rho_{W}^{(0 \mid 1)}(z)=\frac{W^{\prime}(z)-y_{W}(z)}{2}
$$

где

$$
y_{W}^{2}(z)=\left(W^{\prime}(z)\right)^{2}-4 f_{W}^{(0 \mid 1)}(z)
$$


Иногда удобно представить полином $f_{W}^{(0 \mid 1)}(z)$ с помощью $n$ специфических параметров $\widetilde{S}_{i}$ (см. [39]):

$$
4 f_{W}^{(0 \mid 1)}(z)=W^{\prime}(z) \sum_{i=1}^{n} \frac{\widetilde{S}_{i}}{z-\alpha_{i}},
$$

где $\alpha_{i}$ - корни полинома $W^{\prime}(z)=\prod_{i=1}^{n}\left(z-\alpha_{i}\right)$ (так что в правой части соотношения (І.3.46) нет полюсов). Если $\widetilde{S}$ не зависит от параметров $T$, соответствующий ограниченный препотенциал имеет особенно простой вид:

$$
\widetilde{F}^{(0)}[T \mid \widetilde{S}]=-\sum_{i=1}^{n} \widetilde{S}_{i} W\left(\alpha_{i}\right)
$$

На самом деле, поскольку $W^{\prime}\left(\alpha_{i}\right)=0$, второй член в правой части равенства

$$
\frac{\partial \widetilde{F}^{(0)}[T \mid \widetilde{S}]}{\partial T_{k}}=-\sum_{i=1}^{n} \widetilde{S}_{i} \alpha_{i}^{k}+\sum_{i=1}^{n} \frac{k \alpha_{i}^{k}}{M_{i}} \frac{\partial \widetilde{F}^{(0)}[T \mid \widetilde{S}]}{\partial \alpha_{i}}, \quad M_{i} \equiv W^{\prime \prime}\left(\alpha_{i}\right)
$$

сокращается, и действие оператора $\check{R}_{W}(z)$ немедленно переводит первый член в правую часть равенства (І.3.46).

Явные выражения для полиплотностей содержатся в разделе III.

\section{4. О КОЛИЧЕСТВЕ РЕШЕНИЙ}

В этом пункте мы обсудим априорный способ введения фазы и то, насколько естественно их описание, данное выше. Мы также акцентируем внимание на интересной задаче построения матрично-интегральной модели статистической суммы, определенной условиями Вирасоро (I.3.10) или (І.3.20), и обсудим кратко, в каком смысле формулы разложения Гивенталя (которые связаны с матричными интегралами и приводят к статистической сумме КИВ-ДВ) дают решение этой задачи.

Как уже было сказано ранее, существенной составной частью6) теории матричных моделей является утверждение о важности сдвигов переменных $t: Z(t-T)$ и $Z\left(T^{\prime}+t\right)$ с различными $T$ и $T^{\prime}$ могут быть различными ветвями статистической суммы; более того, они ветвятся и далее, давая много (вообще говоря, бесконечно много) подветвей. Ниже мы объясним значение этого утверждения и опишем систему ветвей и их свойства ${ }^{7}$.

\footnotetext{
6) Это новое явление, которое не возникает в простом примере (I.3.1), но возникает уже при рассмотрении интегрального представления цилиндрических функций (I.3.7). В самом деле, контур интегрирования, например для функций Ганкеля, довольно причудлив, так что сдвиг переменной интегрирования переводит любую из функций Ганкеля в их линейную комбинацию.

7) После того как это будет сделано, можно перейти к изучению непрерывных nределов этих ветвей, которые возникают при $N \rightarrow \infty$ и некотором заданном поведении других параметров, таким образом приходя (в зависимости от поведения этого бесконечного множества параметров) к $\infty^{\infty}$ числу типов непрерывных пределов (их может быть довольно мало на нижних ветвях, и только они уже описаны в существующей литературе). Однако ниже мы ограничимся рассмотрением конечных $N$ : классификация ветвей является необходимым условием для классификации непрерывных пределов, и уже она достаточно сложна.
} 
Первый вопрос, к которому следует обратиться, состоит в перечислении ветвей, т.е. различных формальных рядов, являюшихся решениями системы (І.3.10). Как уже упоминалось, сложности обусловлены тем, что ряды зависят от отношений переменных $t$ и различные ветви возникают при различных комбинациях $t$, которые могут находиться в знаменателях. Можно выделить три уровня классификации ветвей, характеризуемых параметрами $g, W(z)=\sum T_{k} z^{k}$ и $f_{W}^{(p \mid m)}\left(z_{1}, \ldots, z_{m} \mid g, T\right)$.

4.1. Типы зависимости от $g$. Зависимость от $g$ характеризует степень сингулярности, возникающую при $t_{k} \rightarrow 0$ или $t_{k} \rightarrow \infty$. С самого начала можно выделить три возможности.

1. Функция $Z$ разлагается по неотрицательным степеням $g$. Лидируюший член разложения в этой фазе описывает “наивный” предел $g=0$ и удовлетворяет уравнению

$$
\sum_{k \geqslant 0} k t_{k} \frac{\partial Z}{\partial t_{k+m}}=0, \quad m \geqslant-1 .
$$

2. Функция $Z$ разлагается по неположительным степеням $g$. В этой фазе лидирующий член разложения соответствует "наивному" пределу $g=\infty$ и удовлетворяет уравнению

$$
\sum_{\substack{a, b \geqslant 0 \\ a+b=m}} \frac{\partial^{2} Z}{\partial t_{a} \partial t_{b}}=0, \quad m \geqslant-1 .
$$

3. Альтернативой по отношению к этим двум ситуациям является разложение в ряд Лорана по степеням $g$ с бесконечным количеством как положительных, так и отрицательных степеней $g$ (если множество степеней полубесконечно, то умножение функции $Z$ на подходяшую степень $g$ переводит ее в один из двух описанных выше классов). Бесконечные ряды Лорана иногда можно дальше разделить на классы, если вместо функции $Z$ рассмотреть препотенииал $\mathcal{F}=g^{2} \ln Z$. Тогда

$$
\mathcal{F}_{q, s}=\sum_{q \leqslant p \leqslant s} g^{2 p} \mathcal{F}_{q, s}^{(p)}
$$

приводит к бесконечному ряду Лорана при разложении $Z$ всякий раз, когда $q \leqslant 0$ и $s \geqslant 2$. Различные пары $(q, s)$ приводят к различным ветвям функции $Z$. На самом деле решения системы (І.3.10) сушествуют, только если $q=-\infty$ или $s=\infty$. Если $q=-\infty$ и $s=\infty$, то трюк можно повторить и рассмотреть, к примеру, $\ln \mathcal{F}$ вместо $\mathcal{F}$, расщепляя "точку" $(-\infty, \infty)$ еще, и так далее. Также $p$ в формуле (I.4.3) может быть, как минимум, полуцелым (более общие разложения по нецелым степеням $t_{k}$ еще более экзотичны, хотя и представляют интерес). Из всех возможных $\mathcal{F}_{q, s}$ в литературе наибольшее внимание уделено случаю $\mathcal{F} \equiv \mathcal{F}_{0, \infty}$ обычно с целыми (а не полуцелыми) $p$. В этом случае разложение (I.4.3) называется разложением по родам. $\mathcal{F}^{(0)}$ является сферическим вкладом в препотенциал $\mathcal{F}=\mathcal{F}_{0, \infty}$, а $p$ указывает род. Если ввести полуцелые $p$, то они будут соответствовать римановым поверхностям с границами или неориентированным поверхностям (в обоих случаях сушествуют дубли, которые являются обыкновенными римановыми поверхностями с целым родом). 
В большинстве фаз для заданных $q$ и $s$ функции $\mathcal{F}_{q, s},\left(\right.$ в частности, $\left.\mathcal{F}_{0, \infty}\right)$ обладают дополнительными свободными параметрами, которые не фиксируются условиями Вирасоро (I.3.10). В п. І.3.4 мы обозначили эти параметры через $f$ (на самом деле функции $f(z)$ получаются из произвольной функции $F[g, T]$ от параметров $T$, см. (I.3.41)). Любая линейная комбинация

$$
Z\{c\}=\sum_{q, s ; f} c_{q, s ; f} \exp \mathcal{F}_{q, s ; f}(g \mid t)
$$

с не зависящими от $t$ (но, возможно, зависяшими от $g$ ) коэффициентами $c_{q, s ; f}$ является решением линейной системы (І.3.10), и коэффициенты $c_{q, s ; f}$ нельзя убрать с помощью переопределения $f$. Эти коэффициенты $c_{q, s ; f}$ являются аналогами коэффициентов $\alpha$ и $\beta$ в примере (I.3.3), и их можно интерпретировать как замену контуров интегрирования циклами с комплексными коэффициентами. В дальнейшем мы не будем уделять внимание свободе, связанной с коэффициентами $c_{q, s ; f}$, и ограничимся рассмотрением генераторов $D$-модуля $Z_{q, s ; f}=\exp \mathcal{F}_{q, s ; f}(g \mid t)$. Нашей следуюшей задачей будет объяснение происхождения свободных параметров ${ }^{8)} f$.

4.2. Сдвиги переменных $t$ и происхождение параметров $f$ : сверхпростой пример. Если зависимость от $g$ установлена, можно перейти к перечислению решений системы (І.3.10). Для того чтобы описать различные фазы, мы сдвигаем переменные $t_{k} \rightarrow-T_{k}+t_{k}$ в предположении, что только первые $n+1$ из параметров $T_{k}$ могут быть ненулевыми.

Чтобы понять, как новые свободные параметры возникают при нетривиальном $W(z)$, поучительно будет начать с "наивного" предела $g=0$, т.е. со статистической суммы, удовлетворяюшей уравнению (I.4.1). Отметим, что она не имеет отношения к статистической сумме в “сферическом" (род $p=0$ ) приближении, $\exp \mathcal{F}^{(0)}$. Вместо формулы (I.3.20) теперь мы имеем для функции

$$
\rho_{0}(z)=\widehat{\nabla}(z) \ln Z
$$

(отметим отсутствие множителя $g^{2}$ в этом определении), взятой при $t_{k}=0$ (т.е. при $v(z)=0)$, следуюшее уравнение:

$$
\widehat{P}_{z}^{-}\left[W^{\prime}(z) \rho_{0}(z)\right]=0, \quad \text { т.e. } \quad \rho_{0}(z)=\frac{f(z)}{W^{\prime}(z)},
$$

где $\operatorname{deg} f(z)<\operatorname{deg} W^{\prime}(z)$, поскольку оператор $\widehat{\nabla}(z)$ и $\rho_{0}(z)$ в (I.4.5) разлагаются по отрицательным степеням $z$. Это означает, что полином $f(z)$ степени $n-1$ является произвольным. А значит, при заданном $W(z)$ степени $n+1$ мы имеем $n$-параметрическое семейство уравнений, даже если все $t_{k}=0$. Включение $t_{k}$ эквивалентно увеличению степени $W(z)$ и, таким образом, может привести к новым произвольным полиномам $f(z)$.

\footnotetext{
${ }^{8)}$ Разделение "степеней свободы" на $f$ и $c_{f}$ не является абсолютно очевидным. Мы описали возможный подход к разделению с помощью базиса в гильбертовом пространстве решений в самом конце введения, более подробно о соответствующем операторе эволюции будет сказано ниже в разделе I.4.3.
} 
Однако коэффициенты этих полиномов в действительности являются функциями параметров $T$, ограниченными дополнительными условиями (I.3.22). Формализм, который будет разработан в п. I.4.3, позволит показать, что все функции $f$ построены из одной произвольной функции $F(T)$ от $n$ переменных $T_{0}, \ldots, T_{n-1}$.

В нашем сверхпростом примере все это, конечно, можно увидеть без применения какого-либо формализма, просто изучая ограниченные условия Вирасоро:

$$
\sum_{k=1}^{n+1} k T_{k} \frac{\partial \mathcal{F}_{W}(t)}{\partial t_{k+m}}=\sum_{k=1}^{\infty} k t_{k} \frac{\partial \mathcal{F}_{W}(t)}{\partial t_{k+m}}, \quad m \geqslant-1 .
$$

Полагая $t_{k}=0$ для всех $k$, мы выражаем все производные $\left.\frac{\partial \mathcal{F}_{W}}{\partial t_{k}}\right|_{t=0}$ c $k \geqslant n$ через производные с $k<n$. Теперь рассмотрим производную по $t_{l}$ от (I.4.7):

$$
\sum_{k=1}^{n+1} k T_{k} \frac{\partial^{2} \mathcal{F}_{W}(t)}{\partial t_{k+m} \partial t_{l}}=l \frac{\partial \mathcal{F}_{W}(t)}{\partial t_{l+m}}+\sum_{k=1}^{\infty} k t_{k} \frac{\partial \mathcal{F}_{W}(t)}{\partial t_{k+m} \partial t_{l}}, \quad m \geqslant-1 .
$$

Положив $t_{k}=0$ для всех $k$, выразим все вторые производные $\left.\frac{\partial^{2} \mathcal{F}_{W}}{\partial t_{k} \partial t_{l}}\right|_{t=0} \mathrm{c} k \geqslant n$ через те, для которых $k<n$. Используя симметрию между $k$ и $l$, приходим к выводу, что из всех вторых производных при $t=0$ только $\left.\frac{\partial^{2} \mathcal{F}_{W}}{\partial t_{k} \partial t_{l}}\right|_{t=0}$ при $k, l<n$ не определяются из условий Вирасоро. Продолжая далее таким же образом, можно доказать, что ограниченные условия Вирасоро определяют все производные, кроме $\left.\frac{\partial^{p} \mathcal{F}_{W}}{\partial t_{k_{1}} \ldots \partial t_{k_{p}}}\right|_{t=0}$ при $k_{1}, \ldots, k_{p}<$ $n$ для любых $p$. Это означает, что единственная свобода, остающаяся после того, как наложены ограниченные условия Вирасоро, заключается в выборе произвольной функции $F[T]=\mathcal{F}_{W}(t=0)$, зависяшей от $n$ переменных $T_{1}, \ldots, T_{n-1}$ (поскольку зависимость от $T_{n}$ и $T_{n+1}$ фиксируется условиями $\hat{L}_{-1}^{W}(t=0) Z_{W}(t=0)=\hat{L}_{0}^{W}(t=0) Z_{W}(t=0)=0$, cм. (I.3.43)).

То же самое должно произойти при $g \neq 0$ с более интересными препотенциалами $\mathcal{F}_{W}^{(p)}$. Они зависят от выбора $W(z)$ и от дополнительных функций типа $f(z)$. Все эти функции, которые становятся все более и более сложными по мере увеличения рода $p$ и при увеличении сложности корреляционных функций (что эквивалентно увеличению числа ненулевых инфинитезимальных переменных $t_{k}$ ), на самом деле построены из производных по параметрам $T$ от одной прозвольной функции переменных $T_{0}, \ldots, T_{n-1}$, т.е. от ограниченного (вычисленного при $t_{k}=0$ ) препотенциала $F(T \mid g)$.

4.3. Оператор эволюции и базис в гильбертовом пространстве решений. В этом пункте мы утверждаем, что для любого $W(z)$, т.е. для любого выбора переменных $T$, сушествует оператор $\breve{U}_{W}(t)$, который переводит любую функцию $Z[T]$ от $T_{0}, \ldots, T_{n-1}$ (с заданной зависимостью от $T_{n}$ и $\left.T_{n+1}\right)$ в функцию $Z_{W}(t)=\check{U}_{W}(t) Z[T]$, которая удовлетворяет условиям Вирасоро $L_{m}^{W}(t) Z_{W}(t)=0, m \geqslant-1$. Более того, если $Z[T]=\sum_{a} c_{a} Z^{(a)}[T]$, то $Z_{W}(t)=\sum_{a} c_{a} Z_{W}^{(a)}(t)$. Построение является непосредственным обобшением построения из п. I.4.2 на случай $g \neq 0$.

Именно, при заданных $T$ мы воспользуемся условиями Вирасоро $\hat{L}_{m}^{W} Z(t)=0$, т.е.

$$
\sum_{k=1}^{n+1} k T_{k} \frac{\partial Z}{\partial t_{k+m}}=\sum_{k=1}^{\infty} k t_{k} \frac{\partial Z}{\partial t_{k+m}}+g^{2} \sum_{a+b=m} \frac{\partial^{2} Z}{\partial t_{a} \partial t_{b}}, \quad m \geqslant-1,
$$


и их кратными производными по $t$, для того чтобы рекуррентно выразить $\left.\frac{\partial^{p} Z}{\partial t_{k_{1}} \ldots \partial t_{k_{p}}}\right|_{t=0}$ при всех $0 \leqslant k_{i}<\infty$ через $\left.g^{2 s} \frac{\partial^{p+s} Z}{\partial T_{l_{1}} \ldots \partial T_{l_{p+s}}}\right|_{t=0}$ при всех $0 \leqslant l_{j}<n$ :

$$
\left.\frac{\partial^{p} Z}{\partial t_{k_{1}} \ldots \partial t_{k_{p}}}\right|_{t=0}=\left.\sum_{\substack{s \\ l_{1}, \ldots, l_{p+s}}} g^{2 s} \mathcal{D}_{k_{1} \ldots k_{p}}^{l_{1} \ldots l_{p+s}} \frac{\partial^{p+s} Z}{\partial T_{l_{1}} \ldots \partial T_{l_{p+s}}}\right|_{t=0}
$$

Это несколько более громоздкая процедура, чем в случае $g=0$, но тем не менее она является явной, и сумма по $s$ конечна, пробегая значения от 0 до целой части от $\left\{\frac{k}{n-1}\right\}$ : выражение для $\left.\frac{\partial Z}{\partial t_{k}}\right|_{t=0}$ содержит $\left.\frac{\partial^{2} Z}{\partial t_{a} \partial t_{b}}\right|_{t=0}$, но только с $a, b \leqslant k-n-1$; далее, выражение для $\left.\frac{\partial^{2} Z}{\partial t_{a} \partial t_{b}}\right|_{t=0}$ содержит $\left.\frac{\partial^{3} Z}{\partial t_{a} \partial t_{b} \partial t_{c}}\right|_{t=0}$ c $a, b, c \leqslant k-2 n-2$ и так далее.

Теперь мы можем определить операторы

$$
\check{D}_{k_{1} \ldots k_{p}}^{(p)}=\left.\sum_{\substack{s \\ 0 \leqslant l_{1}, \ldots, l_{p+s} \leqslant n-1}} g^{2 s} \mathcal{D}_{k_{1} \ldots k_{p}}^{l_{1} \ldots l_{p+s}} \frac{\partial^{p+s}}{\partial T_{l_{1}} \ldots \partial T_{l_{p+s}}}\right|_{t=0} .
$$

Поскольку эти операторы происходят из условий Вирасоро, они удовлетворяют некоторым очевидным соотношениям (уравнениям (I.4.14), см. ниже). Построим теперь оператор эволюции $\check{U}_{W}(t)$ в виде ряда по этим операторам $\check{D}$ :

$$
\check{U}_{W}(t)=1+t_{k} \check{D}_{k}^{(1)}+\frac{1}{2} t_{k} t_{l} \check{D}_{k l}^{(2)}+\frac{1}{6} t_{k} t_{l} t_{m} \check{D}_{k l m}^{(3)}+\cdots .
$$

Тот факт, что $Z[T]$ удовлетворяет уравнениям

$$
\hat{L}_{m}^{W}(t) Z_{W}(t)=\hat{L}_{m}^{W}(t) \check{U}_{W}(t) Z[T]=0,
$$

или просто, что

$$
\begin{aligned}
\hat{L}_{m}^{W}(t) \check{U}_{W}(t)= & \left(\sum_{k} k T_{k} \check{D}_{k+m}^{(1)}+\sum_{a+b=m} \check{D}_{a b}^{(2)}\right)+ \\
& +\sum_{l} t_{l}\left(l \check{D}_{l+m}^{(1)}+\sum_{k} k T_{k} \check{D}_{k+m, l}^{(2)}+\sum_{a+b=m} \check{D}_{a b l}^{(3)}\right)+ \\
& +\frac{1}{2} \sum_{l_{1}, l_{2}} t_{l_{1}} t_{l_{2}}\left(l_{1} \check{D}_{l_{1}+m, l_{2}}^{(2)}+l_{2} \check{D}_{l_{2}+m, l_{1}}^{(2)}+\right. \\
& \left.+\sum_{k} k T_{k} \check{D}_{k+m, l_{1}, l_{2}}^{(3)}+\sum_{a+b=m} \check{D}_{a b l_{1} l_{2}}^{(4)}\right)+\cdots=0
\end{aligned}
$$

эквивалентен утверждению, что все линейные комбинации операторов в скобках обращаются в нуль, и это и есть уравнения на операторы $\check{D}$. Мы доказали, что оператор эволюции один и тот же для всех значений произвольных параметров $f$ (или для любой функции $Z[T])$, если потенциал $W(z)$ фиксирован. Это доказывает, что если $Z[T]=\sum_{a} c_{a} Z^{(a)}[T]$, то $Z_{W}(t)=\sum_{a} c_{a} Z_{W}^{(a)}(t)$, и означает, что “орбиты" оператора эволюции полностью параметризуются потенщиалом $W(z)$. 


\section{II. КОНКРЕТНЫЕ СПЕЦИАЛЬНЫЕ ФУНКЦИИ}

\section{1. ГАУССОВА СТАТИСТИЧЕСКАЯ СУММА}

Гауссова статистическая сумма (фаза) выделена существованием диаграммного представления корреляционных функций и, следовательно, возможностью проинтерпретировать все формулы с помощью комбинаторики ленточных графов. Это делает саму гауссову статистическую сумму важной специальной функцией, даже без учета связи с общей статистической суммой ЭММКР, одной из ветвей которой она является. Более того, формулы разложения типа формулы Гивенталя (см. далее п. II.2) представляют различные ветви как полилинейные комбинации гауссовых статистических сумм. По этим причинам гауссова статистическая сумма $Z_{G}^{M}(t \mid N)$ требует особого внимания, и мы рассматриваем ее отдельно, хотя сушественная часть использованного ниже формализма является частным случаем общего формализма, предназначенного для негауссовых моделей.

Гауссова матричная модель (фаза) соответствует потеншиалу $W(x)=(M / 2) x^{2}$ (т.е. $T_{k}=(M / 2) \delta_{k, 2}$, где $M-$ некоторое число) и седловой точке (см. п.I.2.1) $\phi_{0}=0$,

$$
\begin{aligned}
Z_{G}^{M}(t \mid N) & =\frac{1}{\operatorname{Vol}_{U(N)}} \int D \phi \exp \left(-\frac{M}{2 g} \operatorname{Tr} \phi^{2}+\frac{1}{g} \sum_{k=0}^{\infty} t_{k} \operatorname{Tr} \phi^{k}\right)= \\
& =\left(\frac{2 \pi g}{M}\right)^{N^{2} / 2} \sum_{\vec{k}} \frac{t_{k_{1}} \ldots t_{k_{m}}}{g^{m} m !}\left\langle\operatorname{Tr} \phi^{k_{1}} \cdot \ldots \cdot \operatorname{Tr} \phi^{k_{m}}\right\rangle_{G} .
\end{aligned}
$$

Здесь скобки обозначают среднее по случайным матрищам с гауссовой мерой, $\exp \left\{-(M / 2 g) \operatorname{Tr} \phi^{2}\right\}$.

Каждая корреляционная функция в правой части равенства является суммой по диаграммам Фейнмана-'т Хоофта (ленточных графов) с $m$ вершинами с валентностью $k_{1}, \ldots, k_{m}$. Каждая вершина дает множитель $g^{-1}$, каждый пропагатор, обозначаемый двойной линией (полосой ленточного графа), дает вклад $g / M$, и каждая замкнутая линия (компонента гранишы ленточного графа) дает множитель $N=S / g$, где $S \equiv g N$ это константа связи 'т Хоофта ${ }^{9)}$. Препотенциал $\mathcal{F}_{G}=g^{2} \ln Z_{G}^{M}$ является аналогичной

9) Заметим, что может иметь смысл рассмотреть $S$, зависящие от $g$,

$$
S=\sum_{k=0}^{\infty} S_{k} g^{k}
$$

Однако разложение в формуле (II.1.2) определено для $S=g N$, т.е. можно записать

$$
\mathcal{F}_{G}(t \mid S)=\sum_{p=0}^{\infty} g^{2 p} \widehat{\mathcal{F}}_{G}^{(p)}(t)
$$

$$
\widehat{\mathcal{F}}_{G}^{(0)}(t)=\mathcal{F}_{G}^{(0)}\left(t \mid S_{0}\right), \quad \widehat{\mathcal{F}}_{G}^{(1)}(t)=\mathcal{F}_{G}^{(1)}\left(t \mid S_{0}\right)+S_{1} \frac{\partial}{\partial S_{0}} \mathcal{F}_{G}^{(0)}\left(t \mid S_{0}\right)
$$

и т.д. 
суммой, но только по связныц диаграммам. Если перейти от переменных $N, g$ к переменным $S, g$, то отдельные члены разложения

$$
\mathcal{F}_{G}(t \mid S)=\sum_{p=0}^{\infty} g^{2 p} \mathcal{F}_{G}^{(p)}(t \mid S)
$$

будут суммами по связным ленточным графам рода $p$

$$
\begin{aligned}
\mathcal{F}_{G}^{(p)}(t \mid S) & =F^{(p)}(S)+\sum_{\vec{k}} \frac{t_{k_{1}} \ldots t_{k_{m}}}{m !}\left\langle\left\langle\operatorname{Tr} \phi^{k_{1}} \ldots \cdot \operatorname{Tr} \phi^{k_{m}}\right\rangle\right\rangle^{(p)}= \\
& =F_{G}^{(p)}(S)+\sum_{m \geqslant 1} \frac{1}{m !} \oint \cdots \oint v\left(z_{1}\right) \ldots v\left(z_{m}\right) \rho_{G}^{(p \mid m)}\left(z_{1}, \ldots, z_{m}\right),
\end{aligned}
$$

где $F_{G}^{(p)}(S)$ - это не зависяший от $t$ квазиклассический препотенциал

$$
F_{G}^{(p)}(S)=-\frac{S^{2}}{2} \ln \frac{M}{2 \pi S} \delta_{p, 0}-\left(\ln \operatorname{Vol}_{U(N)}\right)^{(p)}\left(1-\delta_{p, 0}\right)
$$

и гауссовы полиплотности имеют вид

$$
\rho_{G}^{(p \mid m)}\left(z_{1}, \ldots, z_{m}\right)=\left\langle\left\langle T\left(\phi \mid z_{1}\right) \ldots T\left(\phi \mid z_{m}\right)\right\rangle\right\rangle_{G}^{(p)},
$$

где $T(\phi \mid z) \equiv \operatorname{Tr} \frac{1}{z-\phi}$ и $\langle\langle\ldots\rangle\rangle^{(p)}$ обозначают вклад связных диаграмм Фейнмана'т Хоофта рода $p$ в гауссово среднее с весом $\exp \left(-(M / 2) \operatorname{tr} \phi^{2}\right)$, не зависящим от $g$ и $N$. Формально размер матришы теперь равен $S$, а не $N: \operatorname{tr} I=S$ (соответственно мы заменяем обозначение $\operatorname{Tr}$ на tr). Это исчисление оправдывается теоремой Эйлера, утверждающей, что

$$
V-E+F=2-2 h,
$$

где $V$ - количество вершин, $E$ - количество ребер, $F$ - количество граней, $h$-род кривой.

Гауссовы полиплотности $\rho_{G}^{(p \mid m)}$ можно получать рекуррентно по $m$ и затем по $p$, peшая условия Вирасоро (I.3.10), записанные в терминах препотенциалов $\mathcal{F}$,

$$
M \frac{\partial \mathcal{F}_{G}^{(p)}}{\partial t_{m+2}}=\sum_{\substack{a+b=m \\ a, b \geqslant 0}} \sum_{\substack{q+r=p \\ q, r \geqslant 0}} \frac{\partial \mathcal{F}_{G}^{(q)}}{\partial t_{a}} \frac{\partial \mathcal{F}_{G}^{(r)}}{\partial t_{b}}+\sum_{\substack{a+b=m \\ a, b \geqslant 0}} \frac{\partial^{2} \mathcal{F}_{G}^{(p-1)}}{\partial t_{a} \partial t_{b}}+\sum_{k \geqslant 0} k t_{k} \frac{\partial \mathcal{F}_{G}^{(p)}}{\partial t_{k+m}}, \quad m \geqslant-1 .
$$

Суммируя по $m$ с весом $z^{-m-2}$, для производящей функции

$$
\begin{aligned}
\rho_{G}(z \mid t) & =\sum_{k \geqslant 0} \frac{1}{z^{k+1}} \frac{\partial \mathcal{F}_{G}(t \mid \nu)}{\partial t_{k}}=\langle\langle T(\phi \mid z)\rangle\rangle_{G}= \\
& =\sum_{p=0}^{\infty} g^{2 p} \sum_{m \geqslant 0} \frac{1}{m !} \oint \ldots \oint v\left(z_{1}\right) \ldots v\left(z_{m}\right) \rho_{G}^{(p \mid m+1)}\left(z, z_{1}, \ldots, z_{m}\right)
\end{aligned}
$$


получаем уравнение (ср. с (І.3.30))

$$
M\left(z \rho_{G}(z \mid t)-S\right)=\rho_{G}^{2}(z \mid t)+g^{2} \widehat{\nabla}(z) \rho_{G}(z \mid t)+\widehat{P}_{z}^{-}\left[v^{\prime}(z) \rho_{G}(z \mid t)\right],
$$

где

$$
\widehat{P}_{z}^{-}\left[v^{\prime}(z) \rho_{G}(z \mid t)\right]=v^{\prime}(z) \rho_{G}(z \mid t)-\left\langle\left\langle\operatorname{tr} \frac{v^{\prime}(\phi)-v^{\prime}(z)}{\phi-z}\right\rangle\right\rangle_{G},
$$

в то время как

$$
\widehat{P}_{z}^{-}\left[z \rho_{G}(z \mid t)\right]=z \rho_{G}(z \mid t)-\langle\operatorname{tr} I\rangle=z \rho_{G}(z \mid t)-S .
$$

Положив $v(z)=0$ в уравнении (II.1.9), получим для плотностей рекуррентное по $p$ соотношение. Аналогичные рекуррентные соотношения для полиплотностей при $m>1$ можно получить с помощью действия оператора $\widehat{\nabla}\left(z_{1}\right) \ldots \hat{\nabla}\left(z_{m-1}\right)$ на (II.1.9), использовав соотношения (І.3.33) и положив в конще $v(z)=0$. Эта двойная рекуррентная процедура (с рекурсией по $p$ и $m$ ) позволяет получить любую конкретную функцию $\rho^{(p \mid m)}$. Явные выражения для некоторых частных случаев можно найти в разделе III.

Первая (род нуль, одноточечная) плотность, удовлетворяюшая соотношению

$$
M\left(z \rho_{G}^{(0 \mid 1)}(z)-S\right)=\left(\rho_{G}^{(0 \mid 1)}(z)\right)^{2},
$$

- это знаменитое полукруговое распределение (см. [18])

$$
\rho_{G}^{(0 \mid 1)}(z)=\frac{M}{2}\left(z-\sqrt{z^{2}-\frac{4 S}{M}}\right)=\frac{M z-y_{G}(z)}{2}=\sum_{k=0}^{\infty} \frac{c_{k} S^{k+1}}{M^{k} z^{2 k+1}}=\frac{S}{z}+\frac{S^{2}}{M z^{3}}+\cdots
$$

Она оказывается производящей функцией для чисел Каталана

$$
c_{k}=\frac{4^{k} \Gamma(k+1 / 2)}{\Gamma(1 / 2)(k+1) !},
$$

которые задают количества деревьев с $k$ листьями.

Двухточечная плотность удовлетворяет уравнению

$$
\left(M z-2 \rho_{G}^{(0 \mid 1)}(z)\right) \rho_{G}^{(0 \mid 2)}(z, x)=\partial_{x} \frac{\rho_{G}^{(0 \mid 1)}(z)-\rho_{G}^{(0 \mid 1)}(x)}{z-x},
$$

так что

$$
\begin{aligned}
\rho_{G}^{(0 \mid 2)}(z, x) & =\frac{1}{2(z-x)^{2}}\left(-1+\frac{4 M S}{y_{G}(z) y_{G}(x)}\right)= \\
& =\frac{1}{2 x^{2}}\left(-1+\frac{M z}{y_{G}(z)}\right)+\frac{1}{x^{3}} \frac{M z^{2}-2 M S-z y_{G}(z)}{y_{G}(z)}+O\left(\frac{1}{x^{2}}\right) .
\end{aligned}
$$

Первые два члена в правой части равенства являются производяшими функциями для корреляционных функций

$$
\frac{1}{z^{k+1}}\left\langle\left\langle\operatorname{tr} \phi \operatorname{tr} \phi^{k}\right\rangle\right\rangle_{G}^{(0)} \quad \text { и } \quad \frac{1}{z^{k+1}}\left\langle\left\langle\operatorname{tr} \phi^{2} \operatorname{tr} \phi^{k}\right\rangle\right\rangle_{G}^{(0)} .
$$


Вторую из этих производяших функций можно получить другим способом, дифференцируя $\rho_{G}^{(0 \mid 1)}$ по $T_{2}=M / 2$ при условии, что

$$
\frac{\partial S}{\partial T_{2}}=0 \text { для гауссовой модели }
$$

(заметим, что это утверждение неверно для общей ЭММКР из п. I.3.4 даже для квадратичного потенциала $W(z)=M z^{2} / 2$, если $S \neq$ const, т.е. диаграммная техника неприменима даже для квадратичных потенциалов).

Наряду с гауссовыми полиплотностями

$$
\rho_{G}^{(p \mid m)}\left(z_{1}, \ldots, z_{m}\right)=\left\langle\left\langle\operatorname{Tr} \frac{1}{z_{1}-\phi} \ldots \operatorname{Tr} \frac{1}{z_{m}-\phi}\right\rangle\right\rangle_{G}^{(p)}
$$

можно рассматривать их обратные преобразования Лапласа

$$
\begin{aligned}
\eta_{G}^{(p \mid m)}\left(x_{1}, \ldots, x_{m}\right) & =\oint \cdots \oint e^{x_{1} z_{1}+\cdots+x_{m} z_{m}} \rho_{G}^{(p \mid m)}\left(z_{1}, \ldots, z_{m}\right) d z_{1} \ldots d z_{m}= \\
& =\left\langle\left\langle\operatorname{Tr} e^{x_{1} \phi} \ldots \operatorname{Tr} e^{x_{m} \phi}\right\rangle\right\rangle_{G}^{(p)}
\end{aligned}
$$

и их производяшие функции, например

$$
\begin{aligned}
\eta_{G}(x \mid t)= & \oint e^{x z} \rho(z \mid t) d z=\sum_{p, m=0}^{\infty} \frac{g^{2 p}}{m !} \oint \cdots \oint d z d z_{1} \ldots d z_{m} \int_{0}^{\infty} \ldots \int_{0}^{\infty} d x_{1} \ldots d x_{m} \times \\
& \times e^{-x_{1} z_{1}-\ldots-x_{m} z_{m}} \bar{v}\left(z_{1}\right) \ldots \bar{v}\left(z_{m}\right) \eta_{G}^{(p \mid m+1)}\left(x, x_{1}, \ldots, x_{m}\right) .
\end{aligned}
$$

Прямое преобразование Лапласа преобразует функцию $\eta_{G}(x)$ назад в функцию $\rho_{G}(z)$ :

$$
\rho_{G}(z \mid t)=\int_{0}^{\infty} e^{-x z} \eta_{G}(x \mid t) d x
$$

и

$$
\rho_{G}^{(p \mid m)}\left(z_{1}, \ldots, z_{m}\right)=\int_{0}^{\infty} \cdots \int_{0}^{\infty} e^{-x_{1} z_{1}-\ldots-x_{m} z_{m}} \eta_{G}^{(p \mid m)}\left(x_{1}, \ldots, x_{m}\right) d x_{1} \ldots d x_{m} .
$$

Гауссовы (одноточечные) плотности удовлетворяют правилу сумм.м, которое говорит о полном количестве “лепестковых" диаграмм, дающих вклад в $\left\langle\operatorname{Tr} \phi^{2 k}\right\rangle$,

$$
\sum_{p=0}^{\infty} \oint z^{2 k} \rho^{(p \mid 1)}(z)=(2 k-1) ! !\left(\frac{g}{m}\right)^{k}
$$


Правило сумм (II.1.24) является частным случаем (при $N=1$ ) более общего тождества [40], [41], которое задает производящую функцию для всех (для любого рода) гауссовых одноточечных функций (плотностей),

$$
\begin{aligned}
\sum_{k=0}^{\infty} \frac{\left(x^{2} M / g\right)^{k}}{(2 k-1) ! !}\left\langle\operatorname{Tr} \phi^{2 k}\right\rangle_{G}= & \frac{1}{2 x^{2}}\left(\left(\frac{1+x^{2}}{1-x^{2}}\right)^{N}-1\right)=N+N^{2} x^{2}+\frac{\left(2 N^{3}+N\right) x^{4}}{3}+ \\
& +\frac{\left(5 N^{4}+10 N^{2}\right) x^{6}}{15}+\frac{\left(14 N^{5}+70 N^{3}+21 N\right) x^{8}}{105}+ \\
& +\frac{\left(42 N^{6}+420 N^{4}+483 N^{2}\right) x^{10}}{945}+\cdots
\end{aligned}
$$

здесь $(2 k-1) ! !=2^{k} \frac{\Gamma(k+1 / 2)}{\Gamma(1 / 2)}$, так что $(-1) ! !=1$.

Гауссову статистическую сумму $Z_{G}^{M}(t \mid N)$ можно также представить как член семейства моделей Концевича [12]-[17], [6]: она совпадает с гауссовой моделью Концевича с логарифмическим потенциалом [15]

$$
\int d H_{N \times N} \exp \left(-\sum_{k} t_{k} \operatorname{Tr} M^{k}\right)=(2 \pi)^{N^{2} / 2} \frac{\int d X_{n \times n} \operatorname{det}(I-X / H) e^{-\operatorname{Tr} X^{2} / 2}}{\int d X_{n \times n} e^{-\operatorname{Tr} X^{2} / 2}}
$$

где времена $t_{k}$ выражаются через внешнюю матрицу в интеграле Концевича,

$$
t_{k}=\frac{1}{k} \operatorname{Tr} H^{-k}+\frac{1}{2} \delta_{k, 2},
$$

причем размеры матриц $n \times n$ в правой части равенства и $N \times N$ в левой части являются абсолютно независимыми параметрами. Полиплотности $\rho^{(p \mid m)}$ также можно получить из квадратичной модели Конщевича (мы отложим обсуждение этого вопроса до появления отдельной публикации, посвяшенной матричным моделям типа моделей Концевича).

Ожидается, что трехточечные функции для рода нуль, в частности $\rho^{(0 \mid 3)}\left(z_{1}, z_{2}, z_{3}\right)$, удовлетворяют уравнениям Виттена-Дийкграафа-Верлинде-Верлинде (ВДВВ) [42]-[44].

\section{2. ФОРМУЛЫ РАЗЛОЖЕНИЯ ТИПА ФОРМУЛЫ ГИВЕНТАЛЯ ДЛЯ НЕГАУССОВЫХ СТАТИСТИЧЕСКИХ СУММ КИВ-ДВ}

Сдвиг переменных $t$ переводит интеграл (I.2.10) в интеграл

$$
Z_{N, W}^{(\mathrm{matr})}(t)=\frac{1}{\operatorname{Vol}_{U(N)}} \int d \phi \exp \left(-\frac{1}{g} \operatorname{Tr} W(\phi)\right) \exp \left(\frac{1}{g} \sum_{k} t_{k} \operatorname{Tr} \phi^{k}\right) .
$$

На первый взгляд может показаться, что в этом определении нет произвола и свободным параметрам взяться неоткуда. Однако это неверно, если разрешить любые аналитические продолжения по переменным $T$ (коэффициентам $W(\phi)$ ). Один из способов построения решений линейной системы условий Вирасоро (І.3.10) в виде формальных рядов 
(по степеням $t_{k}$ ) состоит в аналитически продолженном методе перевала. Различные генераторы $D$-модуля представляются разложениями вокруг различных седловых точек $\phi=\phi_{0}$ таких, что $W^{\prime}\left(\phi_{0}\right)=0$. Если полином

$$
W^{\prime}(x)=\prod_{i=1}^{n}\left(x-\alpha_{i}\right)
$$

имеет корни $\alpha_{i}$, тогда, поскольку $\phi_{0}$ - это матришы, определенные по модулю $U(N)$-сопряжений (с помошью которых можно диагонализовать любую матрицу и поменять порядок ее собственных значений), различные седловые точки представимы в виде

$$
\phi_{0}=\operatorname{diag}\left(\alpha_{1}, \ldots, \alpha_{1} ; \alpha_{2}, \ldots, \alpha_{2} ; \ldots ; \alpha_{n}, \ldots, \alpha_{n}\right),
$$

где $\alpha_{i}$ появляется $N_{i}$ раз, $\sum_{i=1}^{n} N_{i}=N$. Поскольку мы работаем с $D$-модулем, нет необходимости считать эти $N_{i}$ неотрицательными целыми: в окончательных выражениях (таких как формулы для полиплотностей и препотенциалов) их можно заменить на комплексные числа. Более того, $N_{i}$ могут зависеть от $g$ и от $T_{k}$ (т.е. от формы потенциала $W(\phi))$ с единственными ограничениями, следуюшими из дополнительных условий (I.3.16). Таким образом, ограниченная свобода выбора функций $N_{i}(g, W)$ соответствует свободным параметрам $f$ в $\exp \mathcal{F}_{q, s ; f}$.

Вклад отдельного экстремума $\phi_{0}$ (который характеризуется числами $N_{i}$ ) задается формулой разложения типа формулы Гивенталя ${ }^{10)}$, выражающей его через произведение $n$ гауссовых статистических сумм, каждая со своими $N_{i}$ и $M_{i}=W^{\prime \prime}\left(\alpha_{i}\right)=$ $\prod_{j \neq i}\left(\alpha_{i}-\alpha_{j}\right)$

$$
Z_{N, W}^{(\mathrm{matr})}\left(t \mid \phi_{0}\right)=\frac{\prod_{i=1}^{n} e^{-N_{i} W\left(\alpha_{i}\right)} \operatorname{Vol}_{U\left(N_{i}\right)}}{\operatorname{Vol}_{U(N)}} \prod_{i<j}^{n} \alpha_{i j}^{2 N_{i} N_{j}} \prod_{i<j}^{n} \widehat{\mathcal{O}}_{i j} \prod_{i=1}^{n} \widehat{\mathcal{O}}_{i} \prod_{i=1}^{n} Z_{G}^{M_{i}}\left(t^{(i)} \mid N_{i}\right),
$$

10) Она выводится так же, как в [23] - с помощью перехода от интеграла по собственным значениям матрицы $\phi$ размера $N \times N$ к $n$ интегралам по собственным значениям матриц $\phi_{i}$ с размерами $N_{i} \times N_{i}$ (каждая получается сдвигом на $\alpha_{i} \times I$, это просто замена переменных в интеграле (II.2.1)), а конкретнее - при представлении квадрата определителя Вандермонда в формуле (I.2.8) в виде

$$
\begin{aligned}
\Delta_{N}^{2}(\phi) & \equiv \prod_{a<b}^{N}\left(\phi_{a}-\phi_{b}\right)^{2}=\prod_{i=1}^{n}\left(\prod_{a<b}^{N_{i}}\left(\phi_{i, a}-\phi_{i, b}\right)^{2}\right) \prod_{i<j}^{n}\left(\prod_{a=1}^{N_{i}} \prod_{b=1}^{N_{j}}\left(\alpha_{i j}+\phi_{i, a}-\phi_{j, b}\right)^{2}\right)= \\
& =\prod_{i=1}^{n} \Delta_{N_{i}}^{2}\left(\phi_{i}\right) \prod_{i<j}^{n} \alpha_{i j}^{2 N_{i} N_{j}} \exp \left(2 \sum_{m=1}^{\infty} \frac{(-)^{m-1}}{m \alpha_{i j}^{m}} \operatorname{Tr}_{i} \operatorname{Tr}_{j}\left(\phi_{i} \otimes I_{j}-I_{i} \otimes \phi_{j}\right)^{m}\right)= \\
& =\prod_{i=1}^{n} \Delta_{N_{i}}^{2}\left(\phi_{i}\right) \prod_{i<j}^{n} \alpha_{i j}^{2 N_{i} N_{j}} \exp \left(2 \sum_{k, l=0}^{\infty}(-)^{k} \frac{(k+l-1) !}{\alpha_{i j}^{k+l} k ! l !} \operatorname{Tr}_{i} \phi_{i}^{k} \operatorname{Tr}_{j} \phi_{j}^{l}\right) .
\end{aligned}
$$

Несмотря на техническую простоту такого преобразования, его действительное квантово-теоретико-полевое значение (точное вложение картановской $S U(N) \tau$-функции в $S\left(\bigotimes_{i=1}^{n} U\left(N_{i}\right)\right) \tau$-функцию) остается недостаточно изученным, в частности неизвестно эффективное преобразование непосредственно в терминах матриц (но не в терминах промежуточных интегралов по собственным значениям), из-за чего неясно, каким образом это преобразование обобщается на некартановские модели. 
где операторы имеют вид

$$
\begin{aligned}
\widehat{\mathcal{O}}_{i j} & =\exp \left(2 \sum_{k, l=0}^{\infty}(-)^{k} \frac{(k+l-1) !}{\alpha_{i j}^{k+l} k ! l !} \frac{\partial}{\partial t_{k}^{(i)}} \frac{\partial}{\partial t_{l}^{(j)}}\right), \quad \alpha_{i j}=\alpha_{i}-\alpha_{j} \\
\widehat{\mathcal{O}}_{i} & =\exp \left(-\sum_{k \geqslant 3} \frac{W^{(k)}\left(\alpha_{i}\right)}{k !} \frac{\partial}{\partial t_{k}^{(i)}}\right)
\end{aligned}
$$

$\left(W^{(k)}(x)=\partial_{x}^{k} W(x)\right)$. Соотношение между временами в двух частях равенства (II.2.4) задается формулой

$$
\sum_{k=0}^{\infty} t_{k}\left(\sum_{i=1}^{n} \operatorname{Tr}_{i}\left(\alpha_{i}+\phi_{i}\right)^{k}\right)=\sum_{i=1}^{n}\left(\sum_{k=0}^{\infty} t_{k}^{(i)} \operatorname{Tr}_{i} \phi_{i}^{k}\right)
$$

с произвольными матрицами $\phi_{i}$ размера $N_{i} \times N_{i}$.

Оператор

$$
\widehat{\nabla}(z)=\sum_{k \geqslant 0} \frac{1}{z^{k+1}} \frac{\partial}{\partial t_{k}}
$$

является линейной комбинацией операторов

$$
\begin{aligned}
\widehat{\nabla}(z)^{(i)} & =\sum_{k \geqslant 0} \frac{1}{z^{k+1}} \frac{\partial}{\partial t_{k}^{(i)}}: \\
\widehat{\nabla}(z) & =\sum_{i=1}^{n} \widehat{\nabla}\left(z-\alpha_{i}\right)^{(i)} .
\end{aligned}
$$

Действуя операторами $\widehat{\nabla}(z)$ на формулу разложения (II.2.4), можно выразить негауссовы полиплотности через гауссовы. Отметим, что оператор

$$
\widehat{\nabla}(z)=\sum_{k \geqslant 0} z^{-k-1} \frac{\partial}{\partial t_{k}}
$$

коммутирует с $\widehat{\mathcal{O}}_{i j}$ и $\widehat{\mathcal{O}}_{i}$ и не действует на коэффициенты $M_{i}=W^{\prime \prime}\left(\alpha_{i}\right)=\prod_{j \neq i} \alpha_{i j}$, которые не зависят от времен $t$. Согласно (II.2.7) и (II.2.8) под действием оператора $\widehat{\nabla}(z)$ на отдельную гауссову составляющую

$$
g^{2} \widehat{\nabla}(z) \ln Z_{G}^{\left[M_{i}\right]}\left(t^{(i)} \mid N_{i}\right)=\rho_{G}^{\left[M_{i}\right]}\left(z-\alpha_{i}\right)
$$

возникает соответствуюшая гауссова плотность, но со сдвинутыми аргументами. В результате, например, имеем

$$
\begin{aligned}
\rho_{W}^{\text {(matr) }}(z)= & \widehat{\nabla}(z) \ln Z_{W}^{\text {(matr) }}= \\
& =\frac{\prod_{i<j}^{n} \widehat{\mathcal{O}}_{i j} \prod_{i=1}^{n} \widehat{\mathcal{O}}_{i}\left(\sum_{i=1}^{n} \rho_{G}^{\left[M_{i}\right]}\left(z-\alpha_{i}\right)\right) \prod_{i=1}^{n} Z_{G}^{\left[M_{i}\right]}\left(t^{(i)} \mid N_{i}\right)}{\prod_{i<j}^{n} \widehat{\mathcal{O}}_{i j} \prod_{i=1}^{n} \widehat{\mathcal{O}}_{i} \prod_{i=1}^{n} Z_{G}^{\left[M_{i}\right]}\left(t^{(i)} \mid N_{i}\right)} .
\end{aligned}
$$


Конечно, $\widehat{\mathcal{O}}_{i}$ и $\widehat{\mathcal{O}}_{i j}$ действуют как на $Z_{G}$, так и на $\rho_{G}$. Аналогично выражения для полиплотностей $\rho_{W}\left(z_{1}, \ldots, z_{m}\right)=\widehat{\nabla}\left(z_{1}\right) \ldots \hat{\nabla}\left(z_{m}\right) \ln Z_{W}$ содержат вставки в числители сумм по разбиениям набора $\left\{z_{1}, \ldots, z_{m}\right\}$ на $n$ поднаборов (некоторые из них могут быть пустым множеством, тогда соответствуюшие $\rho$ не имеют аргументов и равны единище)

$$
\sum_{\{m\} \rightarrow\left\{m_{1}\right\}, \ldots,\left\{m_{n}\right\}}\left(\prod_{i=1}^{n} \rho_{G}^{\left[M_{i}\right]}\left(z_{j_{1}}, \ldots, z_{j_{m_{i}}}\right)\right) .
$$

Выражение (II.2.10) и его обобшение с помошью (II.2.11) достаточно сложны. Роль знаменателя в них, как обычно, заключается в том, чтобы избавиться от несвязных диаграмм Фейнмана-'т Хоофта, включая несвязные вклады от различных гауссовых составляюших. Первые вклады в (II.2.11) выглядят следуюшим образом (мы хотим напомнить, что $\rho_{G}(z)=\langle\langle T(z \mid \phi)\rangle\rangle_{G}$, и ввести очевидное обозначение $z_{i}=z-\alpha_{i}$ для сдвинутых переменных $z$ и $\phi_{i}$ для матричных переменных размера $N_{i} \times N_{i}$ ):

$$
\begin{gathered}
\rho_{W}(z)=\rho_{W}^{(\text {matr) }}(z) \\
\rho_{W}^{(\text {matr) }}(z)=\sum_{i}\left\langle\left\langle T\left(z_{i} \mid \phi_{i}\right) \exp \left(-\frac{1}{g} \operatorname{Tr}_{i} \widetilde{W}_{i}\left(\phi_{i}\right)\right)\right\rangle\right\rangle_{G}^{\left[M_{i}, N_{i}\right]}+ \\
+\sum_{j \neq i} \frac{2 N_{j}}{\alpha_{i j}}\left\langle\left\langle T\left(z_{i} \mid \phi_{i}\right) \operatorname{Tr}_{i} \phi_{i} \exp \left(-\frac{1}{g} \operatorname{Tr}_{i} \widetilde{W}_{i}\left(\phi_{i}\right)\right)\right\rangle\right\rangle_{G}^{\left[M_{i}, N_{i}\right]}+\cdots
\end{gathered}
$$

Здесь

$$
\widetilde{W}_{i}\left(\phi_{i}\right) \equiv W\left(\alpha_{i}+\phi_{i}\right)-W\left(\alpha_{i}\right)-\frac{M_{i} \phi_{i}^{2}}{2}
$$

и структуру других корреляшионных функций можно понять, поскольку любой оператор $\widehat{\mathcal{O}}_{i j}$ вносит под знак корреляционной функции ряд

$$
\exp \sum_{k=1}^{\infty}\left((-)^{k-1} \frac{2}{k \alpha_{i j}^{k}} \operatorname{Tr}_{i} \operatorname{Tr}_{j}\left(\phi_{i}-\phi_{j}\right)^{k}\right)=1+\frac{2}{\alpha_{i j}}\left(N_{j} \operatorname{Tr}_{i} \phi_{i}-N_{i} \operatorname{Tr}_{j} \phi_{j}\right)+\cdots .
$$

Лидируюшие вклады в левую и правую части первого равенства в (II.2.12) равны соответственно

$$
\frac{1}{2}\left(W^{\prime}(z)-\sqrt{\left(W^{\prime}(z)\right)^{2}-4 W^{\prime}(z) \sum_{i} \frac{\widetilde{S}_{i}}{z_{i}}}\right)=\sum_{i} \frac{\widetilde{S}_{i}}{z_{i}}+O\left(\widetilde{S}^{2}\right)
$$

(см. (Т.3.44) и (І.3.46)) и

$$
\sum_{i} \frac{M_{i}}{2}\left(z_{i}-\sqrt{z_{i}^{2}-\frac{4 g N_{i}}{M_{i}}}\right)=\sum_{i} \frac{g N_{i}}{z_{i}}+O\left(g^{2} N^{2}\right)
$$

(см. (II.1.13)), т.е. в лидируюшем приближении (ср. с [39])

$$
\widetilde{S}_{i}=g N_{i}+O\left(g^{2} N^{2}\right)=S_{i}+O\left(S^{2}\right) .
$$


Величины $\rho_{W}(z \mid t)$ и $f_{W}(z \mid t)$ очень похожи на нетривиальные гауссовы средние, соответственно:

$$
\langle\langle T(\phi \mid z)\rangle\rangle_{W}=\left\langle\left\langle T(\phi \mid z) e^{-\operatorname{tr} \widetilde{W}(\phi)}\right\rangle\right\rangle_{G}
$$

и

$$
\left\langle\left\langle R_{W}(\phi \mid z)\right\rangle\right\rangle_{W}=\left\langle\left\langle R_{W}(\phi \mid z) e^{-\operatorname{tr} \widetilde{W}(\phi)}\right\rangle\right\rangle_{G}
$$

c

$$
T(\phi \mid z)=\operatorname{Tr} \frac{1}{z-\phi}, \quad R_{W}(\phi \mid z)=\operatorname{Tr} \frac{W^{\prime}(z)-W^{\prime}(\phi)}{z-\phi}
$$

и $\widetilde{W}(z)=W(z)-W(\alpha)-\frac{M}{2}(z-\alpha)^{2}$. Проблема, однако, состоит в том, что эти средние плохо определены, поскольку сушествуют различные седловые точки $\alpha$, которые являются корнями $W^{\prime}(z)$ (эти седловые точки могут быть как минимумами, так и максимумами функции $W(z))$ с различными значениями $M=W^{\prime \prime}(\alpha)$. Выражения (II.2.18), (II.2.19) могут иметь смысл только для определенных $\widetilde{W}$, связанных с одним из корней. Таким образом, они описывают только специальные, пертурбативные ветви статистической суммы, т.е. случай, когда $N_{i}=N$, а все остальные $N_{j}=0$. Для того чтобы описать общие непертурбативные ветви, необходимо вернуться к формулам (II.2.10) и (II.2.11).

Что касается пертурбативных фаз, то в каждой из них можно воспользоваться разложением (II.1.3) для гауссова препотенциала

$$
\mathcal{F}_{G}^{(\cdot)}=F_{G}^{(\cdot)}+\oint v \rho_{G}^{(\cdot \mid 1)}+\frac{1}{2 !} \oint \oint v v \rho_{G}^{(\cdot \mid 2)}+\cdots
$$

и определить разложение для негауссова потенциала с помощью простого сдвига $v(z) \rightarrow$ $-\widetilde{W}(z)+v(z)$, т.е. пересуммируя диаграммы:

$$
\mathcal{F}_{W}^{(\cdot)}=F_{W}^{(\cdot)}+\oint v \rho_{W}^{(\cdot \mid 1)}+\frac{1}{2 !} \oint \oint v v \rho_{W}^{(\cdot \mid 2)}+\cdots,
$$

так что

$$
\begin{aligned}
F_{W}^{(\cdot)} & =F_{G}^{(\cdot)}+\oint \widetilde{W} \rho_{G}^{(\cdot \mid 1)}+\frac{1}{2 !} \oint \oint \widetilde{W} \widetilde{W} \rho_{G}^{(\cdot \mid 2)}+\cdots \\
\rho_{W}^{(\cdot \mid 1)} & =\rho_{G}^{(\cdot \mid 1)}+\oint \widetilde{W} \rho_{G}^{(\cdot \mid 2)}+\frac{1}{2 !} \oint \oint \widetilde{W} \widetilde{W} \rho_{G}^{(\cdot \mid 3)}+\cdots= \\
& =\sum_{k=0}^{\infty} \frac{1}{z^{k+1}}\left\langle\left\langle\operatorname{Tr} \phi^{k} \exp (-\operatorname{Tr} \widetilde{W}(\phi))\right\rangle_{G} \equiv \sum_{k=0}^{\infty} \frac{1}{z^{k+1}}\left\langle\left\langle\operatorname{Tr} \phi^{k}\right\rangle\right\rangle_{W},\right. \\
\rho_{W}^{(\cdot \mid 2)} & =\rho_{G}^{(\cdot \mid 2)}+\oint \widetilde{W} \rho_{G}^{(\cdot \mid 3)}+\frac{1}{2 !} \oint \oint \widetilde{W} \widetilde{W} \rho_{G}^{(\cdot \mid 4)}+\cdots \equiv \\
& \equiv \sum_{k_{1}, k_{2}=0}^{\infty} \frac{1}{z_{1}^{k_{1}+1} z_{2}^{k_{2}+1}}\left\langle\left\langle\operatorname{Tr} \phi^{k_{1}} \operatorname{Tr} \phi^{k_{2}}\right\rangle_{W} .\right.
\end{aligned}
$$




\section{3. ПРЕПОТЕНЦИАЛ КИВ-ДВ}

Как мы уже упоминали в разделе I, для заданного $W$ решения условий Вирасоро, которые обладают разложением по родам, параметризуются произвольной функцией $F[g, T]$ первых $n$ времен $T_{0}, \ldots, T_{n-1}$ и $g$ (зависимость от $T_{n}$ и $T_{N+1}$ предписывается соотношениями (І.3.43)). Чтобы соответствовать произвольным $F[g, T]$, переменные $S_{i}=g N_{i}$ в формулах разложения (II.2.4) должны также быть $T$ - и $g$-зависимыми (хотя их возможные зависимости от $T$ и $g$ жестко ограничены).

Препотенциал КИВ-ДВ [45]-[47] определяется как $F[g, T]$ с постоянныци

$$
S_{i}=\text { const }
$$

Таким образом, он непосредственно задается формулой (II.2.4), но остается неясным, какого рода дополнительные ограничения нужно добавить к условиям Вирасоро (І.3.10), для того чтобы восстановить ограничение (II.3.1). Однако, как обнаружили Р. Дийкграаф и К. Вафа [25], сушествует новое представление, а именно представление в терминах теории Зайберга-Виттена [35], [48]-[52]. Явные выражения для препотенциала КИВ-ДВ

$$
\mathcal{F}_{\mathrm{DV}}[T \mid S]=\sum_{p \geqslant 0} g^{2 p} \mathcal{F}_{\mathrm{DV}}^{(p)}[T \mid S]
$$

можно найти далее в разделе III.

В рамках теории Зайберга-Виттена-Дийкграафа-Вафыне зависимые от $T$ параметры $S_{i}$ можно выразить через $\mathcal{F}_{\mathrm{DV}}^{(0)}$ :

$$
\begin{gathered}
S_{i}=\oint_{A_{i}} y_{W}(z) d z \\
y_{W}^{2}(z)=\left[W^{\prime}(z)\right]^{2}-4 f_{W}^{\mathrm{DV}}(z), \\
f_{W}^{\mathrm{DV}}(z)=\check{R}_{W}(z) \mathcal{F}_{\mathrm{DV}}^{(0)}[T \mid S]=\sum_{k, l \geqslant 0}(k+l+2) T_{k+l+2} z^{k} \frac{\partial \mathcal{F}_{\mathrm{DV}}^{(0)}}{\partial T_{l}}, \\
\frac{\partial \mathcal{F}_{\mathrm{DV}}^{(0)}}{\partial S_{i}}=\oint_{B_{i}} y_{W}(z) d z .
\end{gathered}
$$

Теперь мы хотим перейти к вычислению препотенциала КИВ-ДВ с использованием формул разложения (II.2.4). Начнем с определения действия оператора

$$
\widehat{\mathcal{O}}_{i}=\exp \left(-\sum_{k=3}^{n+1} \frac{W^{(k)}\left(\alpha_{i}\right)}{k !} \frac{\partial}{\partial t_{k}}\right)=\exp \left(-M_{i} \sum_{k=1}^{n-1} \frac{\sigma_{k}^{(i)}}{(k+2)} \frac{\partial}{\partial t_{k+2}}\right)
$$

2 Теоретическая и математическая физика, т. 142, № 3, 2005 г. 
где

$$
\begin{aligned}
& M_{i}=W^{(2)}\left(\alpha_{i}\right)=W^{\prime \prime}\left(\alpha_{i}\right)=(n+1) T_{n+1} \prod_{j \neq i}^{n} \alpha_{i j}, \\
& 2 M_{i} \sigma_{1}^{(i)}=W^{(3)}\left(\alpha_{i}\right)=2 M_{i} \sum_{j \neq i} \frac{1}{\alpha_{i j}} \\
& 6 M_{i} \sigma_{2}^{(i)}=W^{(4)}\left(\alpha_{i}\right)=6 M_{i} \sum_{j<k} \frac{1}{\alpha_{i j} \alpha_{i k}}, \\
& (p+1) ! M_{i} \sigma_{p}^{(i)}=W^{(p+2)}\left(\alpha_{i}\right)=(p+1) ! M_{i} \sum_{\substack{j_{1}<\cdots<j_{p} \\
j_{1}, \ldots, j_{p} \neq i}} \frac{1}{\alpha_{i j_{1}} \ldots \alpha_{i j_{p}}}
\end{aligned}
$$

на отдельную гауссову статистическую сумму $Z_{G}^{M_{i}}\left(t \mid N_{i}\right)$ :

$$
\begin{aligned}
\widehat{\mathcal{O}}_{i} Z_{G}^{M_{i}} & \left.\left(t \mid N_{i}\right)\right|_{t=0}=\left[1+\left(-\frac{W^{(4)}\left(\alpha_{i}\right)}{4 !}\left\langle\operatorname{tr} \phi^{4}\right\rangle_{G}^{M_{i}}+\frac{\left[W^{(3)}\left(\alpha_{i}\right)\right]^{2}}{2 !(3 !)^{2}}\left\langle\left(\operatorname{tr} \phi^{3}\right)^{2}\right\rangle_{G}^{M_{i}}\right)+\right. \\
& +\left(-\frac{W^{(6)}\left(\alpha_{i}\right)}{6 !}\left\langle\operatorname{tr} \phi^{6}\right\rangle_{G}^{M_{i}}+\frac{W^{(3)}\left(\alpha_{i}\right) W^{(5)}\left(\alpha_{i}\right)}{3 ! 5 !}\left\langle\operatorname{tr} \phi^{3} \operatorname{tr} \phi^{5}\right\rangle_{G}^{M_{i}}+\right. \\
& +\frac{\left[W^{(4)}\left(\alpha_{i}\right)\right]^{2}}{2 !(4 !)^{2}}\left\langle\left(\operatorname{tr} \phi^{4}\right)^{2}\right\rangle_{G}^{M_{i}}-\frac{\left[W^{(3)}\left(\alpha_{i}\right)\right]^{2} W^{(4)}\left(\alpha_{i}\right)}{2 !(3 !)^{2} 4 !}\left\langle\left(\operatorname{tr} \phi^{3}\right)^{2} \operatorname{tr} \phi^{4}\right\rangle_{G}^{M_{i}}+ \\
& \left.+\frac{\left[W^{(3)}\left(\alpha_{i}\right)\right]^{4}}{4 !(3 !)^{4}}\left\langle\left(\operatorname{tr} \phi^{3}\right)^{4}\right\rangle_{G}^{M_{i}}\right)+ \\
& +\left(-\frac{W^{(8)}\left(\alpha_{i}\right)}{8 !}\left\langle\operatorname{tr} \phi^{8}\right\rangle_{G}^{M_{i}}+\frac{W^{(3)}\left(\alpha_{i}\right) W^{(7)}\left(\alpha_{i}\right)}{3 ! 7 !}\left\langle\operatorname{tr} \phi^{3} \operatorname{tr} \phi^{7}\right\rangle_{G}^{M_{i}}+\right. \\
& +\frac{W^{(4)}\left(\alpha_{i}\right) W^{(6)}\left(\alpha_{i}\right)}{4 ! 6 !}\left\langle\operatorname{tr} \phi^{4} \operatorname{tr} \phi^{6}\right\rangle_{G}^{M_{i}}+\frac{\left[W^{(5)}\left(\alpha_{i}\right)\right]^{2}}{2 !(5 !)^{2}}\left\langle\left(\operatorname{tr} \phi^{5}\right)^{2}\right\rangle_{G}^{M_{i}}- \\
& -\frac{\left[W^{(3)}\left(\alpha_{i}\right)\right]^{2} W^{(6)}\left(\alpha_{i}\right)}{2 !(3 !)^{2} 4 !}\left\langle\left(\operatorname{tr} \phi^{3}\right)^{2} \operatorname{tr} \phi^{6}\right\rangle_{G}^{M_{i}}-\frac{\left[W^{(4)}\left(\alpha_{i}\right)\right]^{3}}{3 !(4 !)^{3}}\left\langle\left(\operatorname{tr} \phi^{4}\right)^{3}\right\rangle_{G}^{M_{i}}+ \\
& \left.\left.+\frac{\left[W^{(3)}\left(\alpha_{i}\right)\right]^{3} W^{(5)}\left(\alpha_{i}\right)}{3 !(3 !)^{3} 5 !}\left\langle\left(\operatorname{tr} \phi^{3}\right)^{3} \operatorname{tr} \phi^{5}\right\rangle_{G}^{M_{i}}\right)+\cdots\right]\left.Z_{G}^{M_{i}}\left(t \mid N_{i}\right)\right|_{t=0}
\end{aligned}
$$

Этот формальный ряд становится плохо определенным, когда некоторые $\alpha_{i j}=0$, т.е. когда мы рассматриваем разложение вокруг вырожденного экстремума $\alpha_{i}=\alpha_{j}$. В таких случаях (для таких $W(z))$ нужно применять другой, сушественно негауссов формализм, используюший выражения типа (для $N=1)^{11)}$

$$
\int_{C} d \phi \exp \left(-h \phi^{p}+\sum_{k=1}^{\infty} t_{k} \phi^{k}\right)=\sum_{k \geqslant 0} \frac{\operatorname{Sh}_{k}(t)}{p h^{\frac{k+1}{p}}} \Gamma_{C(p)}\left(\frac{k+1}{p}\right),
$$

\footnotetext{
11) Контуры интегрирования под знаком суммы зависят от $p$ из-за замены переменной интегрирования, которая зависит от $p$.
} 
где $\mathrm{Sh}_{k}(t)$ - это полиномы Шура

$$
\exp \left(\sum_{k=1}^{\infty} t_{k} \phi^{k}\right)=\sum_{k=0}^{\infty} \operatorname{Sh}_{k}(t) \phi^{k}
$$

и гамма-функция для цепи $C$ определяется равенством

$$
\Gamma_{C}(z)=\int_{C} \phi^{z-1} e^{-\phi} d \phi
$$

Обобщение этого вычисления на случай $N \neq 1$ осуществляется с помощью негауссовых полиплотностей $\rho$ для $W(z)=W_{p}(z)=h z^{p}$. Отметим, что связь цепи $C$ с функцией $F_{W_{p}}$ и полиномами $f_{W_{p}}$ достаточно нетривиальна: например, наивный выбор $f^{(0 \mid 1)}=$ $\nu=$ const приводит к тому, что корреляционная функция

$$
\left\langle\operatorname{tr} \phi^{k}\right\rangle_{W_{p}}^{(0)}=\oint_{\infty} z^{k} \rho_{W_{p}}^{(0 \mid 1)}(z \mid f=\text { const }) d z=-\frac{1}{2} \oint_{\infty} z^{k} \sqrt{p^{2} z^{2 p-2}-4 S} d z
$$

не обрашается в нуль, только если $k+1$ является нечетны. $м$ кратным $p-1$, т.е. если $k=$ $(2 n-1)(p-1)-1$, хотя для $N=1$ она должна равняться $\Gamma_{C}\left(\frac{k+1}{p}\right)$. (В гауссовом случае $p=2$ это ограничение на $C$ заключается в том, что $\Gamma_{C}(z) \neq 0$ только при полуцелых, а не при целых значениях аргумента.) Отметим также, что при $p \neq 2$ выбор $f^{(0 \mid 1)}=\nu=$ const никоим образом не является выделенным, поскольку не сушествует $M$ и поэтому естественного определения параметра $\nu$ (который в гауссовом случае равен $g N / M$ ).

В дальнейшем мы будем рассматривать невырожденную ситуацию, когда все $\alpha_{i j} \neq 0$.

При использовании формулы разложения (II.2.4) даже при $t=0$ члены вида

$$
\frac{W^{(3)}\left(\alpha_{i}\right)}{3 !} \frac{\partial}{\partial t_{3}}
$$

в $\widehat{\mathcal{O}}_{W}\left(\alpha_{i}\right)$, которые не дают вклада в (II.3.6), нужно учитывать, поскольку в комбинации с $\widehat{\mathcal{O}}_{i j}$ они могут дать нетривиальные вклады даже при $t=0$.

Для использования формул (II.2.4) при явных вычислениях мы введем двойную градуировку препотенциала - по степеням $g$ и $S$ :

$$
\mathcal{F}=\sum_{p \geqslant 0} \mathcal{F}^{(p)}=\sum_{\substack{p \geqslant 0 \\ m \geqslant 1}} \mathcal{F}^{(p \mid m)}+\widetilde{\mathcal{F}}=\sum_{m \geqslant 1} \mathcal{F}_{m}+\widetilde{\mathcal{F}}
$$

где $\mathcal{F}^{(p \mid m)}$ и $\mathcal{F}_{m}$ обозначают вклады с $m$-й степенью по параметрам $S$ для рода $p$ и всех родов, соответственно. $\widetilde{\mathcal{F}}$ - это вклад, сингулярный по $S$, вид которого можно извлечь из формулы (І.2.9). Явные выражения (которые содержат конечное количество членов) можно найти для каждой конкретной величины $\left.\mathcal{F}(p \mid m)\right|_{t=0}$. Формулы разложения (II.2.4) выражают $\left.\mathcal{F}_{\mathrm{DV}}^{(p \mid m)}\right|_{t=0}$ через $\mathcal{F}_{G}^{\left(p^{\prime} \mid m^{\prime}\right)}$ при $p^{\prime} \leqslant p, m^{\prime} \leqslant m$ и некоторых 
$t \neq 0$; в конечном итоге времена, входящие в потенциал, нужно положить равными константам,

$$
t_{k}^{(i)}=-T_{k}^{(i)}=-\frac{M_{i} \sigma_{k-2}^{(i)}}{k}, \quad 3 \leqslant k \leqslant n+1,
$$

но перед этим нужно взять по этим временам производные.

Формула разложения (II.2.4) гласит, что

$$
\exp \left(\left.\frac{1}{g^{2}} \mathcal{F}_{\mathrm{DV}}\right|_{t=0}\right)=\left.\exp \left(\frac{1}{g^{2}} \mathcal{F}_{\mathrm{DV}}^{\text {pert }}\right) \exp (-(S \partial)) \exp \left(g^{2}(c \partial \partial)\right) \exp \left(\frac{1}{g^{2}} F_{G}\right)\right|_{t_{k}^{(i)}=-T_{k}^{(i)}},
$$

где мы используем следуюшие обозначения: для "нормировочной” части препотенциала -

$$
\exp \left(\frac{1}{g^{2}} \mathcal{F}_{\mathrm{DV}}^{\mathrm{pert}}\right)=\frac{1}{\prod_{i} \operatorname{Vol} U\left(S_{i} / g\right)} \prod_{i} \frac{e^{-S_{i} W\left(\alpha_{i}\right)}}{M_{i}^{S_{i}^{2} / 2 g^{2}}} \prod_{i<j} \alpha_{i j}^{2 S_{i} S_{j} / g^{2}}
$$

для суммы гауссовых препотенциалов -

$$
F_{G}=\sum_{m \geqslant 1} F_{m}=\sum_{i=1}^{n} \mathcal{F}_{G}^{M_{i}}\left(t^{(i)}\right)
$$

и для операторов -

$$
\begin{aligned}
(S \partial) & =\sum_{i \neq j}^{n}\left(\sum_{k \geqslant 1}^{\infty} \frac{2 S_{i}}{k \alpha_{i j}^{k}} \frac{\partial}{\partial t_{k}^{(j)}}\right), \\
(c \partial \partial) & =\sum_{i \neq j}^{n}\left(\sum_{k, l \geqslant 1}^{\infty} \frac{(-)^{k+1}(k+l-1) !}{k ! l ! \alpha_{i j}^{k+l}} \frac{\partial}{\partial t_{k}^{(i)}} \frac{\partial}{\partial t_{l}^{(j)}}\right) .
\end{aligned}
$$

Отметим, что оператор ( $c \partial \partial)$ обращает в нуль любой отдельный препотенциал $F_{m}$ и действует нетривиально только на билинейные комбинации $F$ (и комбинации старших степеней).

Подставляя (см. [23]) формулу

$$
\begin{aligned}
\ln (\operatorname{Vol} U(S / g))= & -\frac{S^{2}}{2 g^{2}} \ln \left(\frac{S}{g}\right)+\frac{1}{12} \ln \left(\frac{S}{g}\right)+\frac{3 S^{2}}{4 g^{2}}- \\
& -\frac{S}{2 g} \ln (2 \pi)-\zeta^{\prime}(-1)-\sum_{k=2}^{\infty} \frac{B_{2 k}}{4 k(k-1)} \frac{g^{2 k-2}}{S^{2 k-2}}
\end{aligned}
$$


в (II.3.13), получаем (разлагая экспоненты в обеих частях равенства и сравнивая коэффициенты при одинаковых степенях $S$ и $g^{2}$ )

$$
\begin{aligned}
\left.\mathcal{F}_{1}^{\mathrm{DV}}\right|_{t=0}= & -\sum_{i} S_{i} W\left(\alpha_{i}\right)+\left.F_{1}\right|_{t_{k}^{(i)}=-T_{k}^{(i)}} \\
\left.\mathcal{F}_{2}^{\mathrm{DV}}\right|_{t=0}= & -\frac{1}{2} \sum_{i<j}\left(S_{i}^{2}-4 S_{i} S_{j}+S_{j}^{2}\right) \ln \alpha_{i j}-\frac{3}{4} \sum_{i} S_{i}^{2}+ \\
& +\left[F_{2}-(S \partial) F_{1}+\frac{1}{2} \sum_{k \geqslant 1}^{\infty} \frac{g^{2 k-2}}{k !}(c \partial \partial)^{k} F_{1}^{2}\right]_{t_{k}^{(i)}=-T_{k}^{(i)}} \\
\left.\mathcal{F}_{3}^{\mathrm{DV}}\right|_{t=0}= & {\left[F_{3}-(S \partial) F_{2}+\frac{1}{2}(S \partial)^{2} F_{1}+\right.} \\
& +\sum_{k \geqslant 1}^{\infty} \frac{g^{2 k-2}}{k !}(c \partial \partial)^{k}\left[F_{1} F_{2}-F_{1}(S \partial) F_{1}\right]+ \\
& \left.+\frac{1}{6} \sum_{k \geqslant 2}^{\infty} \frac{g^{2 k-4}}{k !}\left((c \partial \partial)^{k} F_{1}^{3}-3 F_{1}(c \partial \partial)^{k} F_{1}^{2}\right)\right]_{t_{k}^{(i)}=-T_{k}^{(i)}}
\end{aligned}
$$

В разделе III представлены некоторые старшие члены разложения.

В суммах, оставшихся в формулах (II.3.18), вклад в $\left.\mathcal{F}_{\mathrm{DV}}^{(p \mid m)}\right|_{t=0}$ дает только конечное число членов с заданными $p$ и $m$, причем необходимо учесть нетривиальную зависимость от $g^{2}$ выражения $F_{m}=\sum_{i} F_{m}\left(S_{i} \mid t^{(i)}\right)$ :

$$
\begin{aligned}
F_{m}(S \mid t)= & S^{m} \sum_{p \geqslant 0}^{\infty} g^{2 p}\left[\sum_{s=1}^{\infty} \sum_{\substack{k_{1}<\cdots<k_{s} \\
l_{1}, \ldots, l_{s}}}^{\infty} \frac{t_{k_{1}}^{l_{1}} \ldots t_{k_{s}}^{l_{s}}}{l_{1} ! \ldots l_{s} !}\left\langle k_{1}^{l_{1}}, \ldots, k_{s}^{l_{s}}\right\rangle\right\rangle^{(p)} \times \\
& \left.\times \frac{1}{M^{\frac{1}{2}\left(l_{1} k_{1}+\cdots+l_{s} k_{s}\right)}} \delta\left(\frac{1}{2} \sum_{j=1}^{s} l_{j}\left(k_{j}-2\right)=m+2 p-2\right)\right] .
\end{aligned}
$$

Здесь мы ввели краткое обозначение для комбинаторных величин (задаюших число соответствующих ленточных графов)

$$
\left.\left\langle\left\langle k_{1}^{l_{1}}, \ldots, k_{s}^{l_{s}}\right\rangle\right\rangle^{(p)}=M^{\frac{1}{2}\left(l_{1} k_{1}+\cdots+l_{s} k_{s}\right)}\left\langle\left(\operatorname{tr} \phi^{k_{1}}\right)^{l_{1}} \ldots\left(\operatorname{tr} \phi^{k_{s}}\right)^{l_{s}}\right\rangle\right\rangle_{G}^{(p)} .
$$

Дельта-функция в формуле (II.3.19) ограничивает возможное число ненулевых $t_{k}=T_{k}$, $3 \leqslant k \leqslant n+1$, при заданных $m, p, t_{1}$ и $t_{2}$. Однако, поскольку мы в конце концов собираемся положить $t_{1}=0$ и $t_{2}=0$, их необходимо устранить, применяя операторы $(S \partial)$ и $(c \partial \partial)$, которые дадут дополнительные степени $S$ и $g^{2}$, соответственно. Таким образом, только конечное количество членов в разложении (II.3.19) дает вклад в заданную величину $\left.\mathcal{F}_{\mathrm{DV}}^{(p \mid m)}\right|_{t=0}$. Для изучения этих правил отбора полезно ввести обозначение $\mathcal{N}_{k_{j}}$ для $l_{j}$ в формуле (II.3.20). Тогда дельта-функция в формуле (II.3.19) задает следующее условие: должно вьполняться равенство

$$
\sum_{k=3}^{\infty}(k-2) \mathcal{N}_{k}=\mathcal{N}_{1}+2 m+4 p-4
$$


для того чтобы корреляционная функция

$$
\left\langle\left\langle\left\{\mathcal{N}_{1}, \mathcal{N}_{2}, \ldots\right\}\right\rangle\right\rangle=\left\langle\left\langle(\operatorname{tr} \phi)^{\mathcal{N}_{1}}\left(\operatorname{tr} \phi^{2}\right)^{\mathcal{N}_{2}} \ldots\right\rangle\right\rangle
$$

не обращалась в нуль.

Рассмотрим, например, $m=1$. Поскольку

$$
\left.\mathcal{F}_{1}^{\mathrm{DV}}\right|_{t=0}+\sum_{i} S_{i} W\left(\alpha_{i}\right)=\left.F_{1}\right|_{t_{k}^{(i)}=-T_{k}^{(i)}}
$$

и в формуле (II.3.19)

$$
\sum_{k=3}^{\infty}(k-2) \mathcal{N}_{k}=\mathcal{N}_{1}+4 p-2
$$

то при $p=0$ в правой части равенства (II.3.20) нет ненулевых членов, в то время как при $p=1$ мы имеем условие $2 \mathcal{N}_{4}+\mathcal{N}_{3}=2$ (т.е. либо $\mathcal{N}_{4}=1$, либо $\mathcal{N}_{3}=2$ ), а при $p=2$ условие имеет вид $6 \mathcal{N}_{8}+5 \mathcal{N}_{7}+4 \mathcal{N}_{6}+3 \mathcal{N}_{5}+2 \mathcal{N}_{4}+\mathcal{N}_{3}=6$. Таким образом, для вкладов нулевого и первого порядков имеем следующие явные выражения:

$$
\begin{aligned}
\left.\mathcal{F}_{\mathrm{DV}}^{(0 \mid 1)}\right|_{t=0} & =-\sum_{i} S_{i} W\left(\alpha_{i}\right), \\
\left.\mathcal{F}_{\mathrm{DV}}^{(1 \mid 1)}\right|_{t=0} & =\sum_{i} \frac{S_{i}}{M_{i}}\left(-\frac{\left\langle\left\langle 4^{1}\right\rangle\right\rangle^{(1)}}{4} \sigma_{2}^{(i)}+\frac{\left\langle\left\langle 3^{2}\right\rangle\right\rangle^{(1)}}{2 ! \cdot 3^{2}}\left[\sigma_{1}^{(i)}\right]^{2}\right)= \\
& =\sum_{i} \frac{S_{i}}{M_{i}}\left(-\frac{\sigma_{2}^{(i)}}{4}+\frac{3\left[\sigma_{1}^{(i)}\right]^{2}}{2 ! \cdot 3^{2}}\right)=\sum_{i} \frac{S_{i}}{12 M_{i}}\left(2\left[\sigma_{1}^{(i)}\right]^{2}-3 \sigma_{2}^{(i)}\right) .
\end{aligned}
$$

Другие явные выражения представлены в разделе III.

\section{III. ТАБЛИЦЫ}

\section{1. ГАУССОВА СТАТИСТИЧЕСКАЯ СУММА}

1.1. Первые обобщенные числа Каталана. В таблице представлено количество графов рода $p$, построенных по одной $2 k$-валентной вершине, т.е. коэффициенты раз- 
ложения коррелятора $\left\langle\operatorname{Tr} \phi^{2 k}\right\rangle$ по родам $(m=(2 k-1) ! !$ - общее количество $2 k$-валентных графов):

\begin{tabular}{|c|c|cccccc|}
\hline \multirow{2}{*}{$2 k$} & \multirow{2}{*}{$m$} & \multicolumn{7}{|c|}{$p$} & \\
\cline { 2 - 7 } & & 0 & 1 & 2 & 3 & 4 & 5 \\
\hline 0 & 1 & 1 & - & - & - & - & - \\
2 & 1 & 1 & - & - & - & - & - \\
4 & 3 & 2 & 1 & - & - & - & - \\
6 & 15 & 5 & 10 & - & - & - & - \\
8 & 105 & 14 & 70 & 21 & - & - & - \\
10 & 945 & 42 & 420 & 483 & - & - & - \\
12 & $945 \cdot 11$ & 132 & 2310 & 6468 & 1485 & - & - \\
14 & $945 \cdot 11 \cdot 13$ & 429 & 12012 & 66066 & 56628 & - & - \\
16 & $945 \cdot 11 \cdot 13 \cdot 15$ & 1430 & 60060 & 570570 & 1169740 & 225225 & - \\
18 & $945 \cdot 11 \cdot 13 \cdot 15 \cdot 17$ & 4862 & 291720 & 4390386 & 17454580 & 12317877 & - \\
20 & $945 \cdot 11 \cdot 13 \cdot 15 \cdot 17 \cdot 19$ & 16796 & 1385670 & 31039008 & 211083730 & 351683046 & 59520825 \\
\hline
\end{tabular}

1.2. Первые гауссовы плотности (производящие функции обобщенных чисел Каталана); $\nu=g N / M$.

Род $p=0$ :

$$
\frac{1}{M} \rho^{(0 \mid 1)}(z)=\frac{z-y(z)}{2}=\sum_{k=0}^{\infty} \frac{4^{k} \Gamma(k+1 / 2)}{\Gamma(1 / 2)(k+1) !} \frac{\nu^{k+1}}{z^{2 k+1}}
$$

где

$$
y^{2}(z)=y_{G}^{2}(z)=z^{2}-4 \nu, \quad \nu=\frac{g N}{M}=\frac{S}{M} .
$$

Род $p=1$ :

$$
M \rho^{(1 \mid 1)}(z)=\frac{\nu}{y^{5}(z)}=\sum_{k=0}^{\infty} \frac{4^{k} \Gamma(k+5 / 2)}{\Gamma(5 / 2) k !} \frac{\nu^{2 k+1}}{z^{2 k+5}} .
$$

Род $p=2$ :

$$
\begin{aligned}
M^{3} \rho^{(2 \mid 1)}(z) & =\frac{21 \nu\left(z^{2}+\nu\right)}{y^{11}(z)}=21 \sum_{k=0}^{\infty}\left(\frac{4^{k} \Gamma(k+11 / 2)}{\Gamma(11 / 2) k !}+\frac{4^{k-1} \Gamma(k+9 / 2)}{\Gamma(11 / 2)(k-1) !}\right) \frac{\nu^{2 k+1}}{z^{2 k+9}}= \\
& =\frac{\nu}{z^{9}}+\frac{14}{3} \sum_{k=1}^{\infty} \frac{4^{k-1} \Gamma(k+9 / 2)(5 k+18)}{\Gamma(9 / 2)(k-1) !} \frac{\nu^{2 k+1}}{z^{2 k+9}} .
\end{aligned}
$$


Род $p=3$ :

$$
\begin{aligned}
M^{5} \rho^{(3 \mid 1)}(z)= & \frac{11 \nu\left(135 z^{4}+558 \nu z^{2}+158 \nu^{2}\right)}{y^{17}(z)}= \\
= & 11 \sum_{k=0}^{\infty}\left(135 \cdot \frac{4^{k} \Gamma(k+17 / 2)}{\Gamma(17 / 2) k !}+558 \cdot \frac{4^{k-1} \Gamma(k+15 / 2)}{\Gamma(17 / 2)(k-1) !}+\right. \\
& \left.+158 \cdot \frac{4^{k-2} \Gamma(k+13 / 2)}{\Gamma(17 / 2)(k-2) !}\right) \frac{\nu^{2 k+1}}{z^{2 k+13}}= \\
= & \frac{\nu}{z^{13}}+\frac{\nu^{2}}{z^{15}}+\frac{44}{13 \cdot 15} \sum_{k=2}^{\infty} \frac{4^{k-2} \Gamma(k+13 / 2)\left(a k^{2}+b k+c\right)}{\Gamma(13 / 2)(k-1) !} \frac{\nu^{2 k+1}}{z^{2 k+13}}, \\
a k^{2}+b k+c= & 135 \cdot 16(k+13 / 2)(k+15 / 2)+558 \cdot 4 k(k+15 / 2)+158 k(k-1) .
\end{aligned}
$$

Произвольный род $p>0$ :

$$
M^{2 p-1} \cdot \rho^{(p \mid 1)}(z)=\frac{\nu Q_{p-1}\left(z^{2} \mid \nu\right)}{y^{6 p-1}(z)}
$$

где

$$
Q_{p-1}\left(z^{2} \mid \nu\right)=\frac{(4 p-1) ! !}{2 p+1} z^{2 p-2}+\cdots
$$

- это полином степени $p-1$ по $z^{2}$ :

$$
\begin{aligned}
Q_{0}\left(x^{2} \mid \nu\right)= & \\
Q_{1}\left(x^{2} \mid \nu\right)= & 21\left(x^{2}+\nu\right) \\
Q_{2}\left(x^{2} \mid \nu\right)= & 11\left(135 x^{4}+558 x^{2} \nu+158 \nu^{2}\right), \\
Q_{3}\left(x^{2} \mid \nu\right)= & 143\left(1575 x^{6}+13689 x^{4} \nu+18378 x^{2} \nu^{2}+2339 \nu^{3}\right), \\
Q_{4}\left(x^{2} \mid \nu\right)= & 88179\left(675 x^{8}+9660 x^{6} \nu+28764 x^{4} \nu^{2}+18908 x^{2} \nu^{3}+1354 \nu^{4}\right), \\
Q_{5}\left(x^{2} \mid \nu\right)= & 111435\left(218295 x^{10}+4534875 x^{8} \nu+23001156 x^{6} \nu^{2}+34604118 x^{4} \nu^{3}+\right. \\
& \left.+13447818 x^{2} \nu^{4}+617926 \nu^{5}\right) .
\end{aligned}
$$

\section{3. Первые рекуррентные соотношения для гауссовых полиплотностей:}

$$
\begin{aligned}
y\left(z_{1}\right) \hat{\rho}^{(0 \mid 2)}\left(z_{1}, z_{2}\right)= & \partial_{z_{2}} \frac{\hat{\rho}^{(0 \mid 1)}\left(z_{1}\right)-\hat{\rho}^{(0 \mid 1)}\left(z_{2}\right)}{z_{1}-z_{2}} \\
y\left(z_{1}\right) \hat{\rho}^{(1 \mid 1)}\left(z_{1}\right)= & \hat{\rho}^{(0 \mid 2)}\left(z_{1}, z_{1}\right) \\
y\left(z_{1}\right) \hat{\rho}^{(0 \mid 3)}\left(z_{1}, z_{2}, z_{3}\right)= & 2 \hat{\rho}^{(0 \mid 2)}\left(z_{1}, z_{2}\right) \hat{\rho}^{(0 \mid 2)}\left(z_{1}, z_{3}\right)+\partial_{z_{2}} \frac{\hat{\rho}^{(0 \mid 2)}\left(z_{1}, z_{3}\right)-\hat{\rho}^{(0 \mid 2)}\left(z_{2}, z_{3}\right)}{z_{1}-z_{2}}+ \\
& +\partial_{z_{3}} \frac{\hat{\rho}^{(0 \mid 2)}\left(z_{1}, z_{2}\right)-\hat{\rho}^{(0 \mid 2)}\left(z_{2}, z_{3}\right)}{z_{1}-z_{3}}, \\
y\left(z_{1}\right) \hat{\rho}^{(1 \mid 2)}\left(z_{1}, z_{2}\right)= & 2 \hat{\rho}^{(0 \mid 2)}\left(z_{1}, z_{2}\right) \hat{\rho}^{(1 \mid 1)}\left(z_{1}\right)+\hat{\rho}^{(0 \mid 3)}\left(z_{1}, z_{1}, z_{2}\right)+ \\
& +\partial_{z_{2}} \frac{\hat{\rho}^{(1 \mid 1)}\left(z_{1}\right)-\hat{\rho}^{(1 \mid 1)}\left(z_{2}\right)}{z_{1}-z_{2}}, \\
y\left(z_{1}\right) \hat{\rho}^{(2 \mid 1)}\left(z_{1}\right)= & \left(\hat{\rho}^{(1 \mid 1)}\left(z_{1}\right)\right)^{2}+\hat{\rho}^{(1 \mid 2)}\left(z_{1}, z_{1}\right) .
\end{aligned}
$$


1.4. Первые гауссовы полиплотности: аналитические выражения; $\nu=$ $g N / M, y(z)=z^{2}-4 \nu$. Первые гауссовы полиплотности (одно-, двух-, трех-, ... точечные функции).

Род $p=0$ :

$$
\begin{aligned}
\frac{1}{M} \rho^{(0 \mid 1)}(z) & =\frac{z-y(z)}{2}, \\
\rho^{(0 \mid 2)}\left(z_{1}, z_{2}\right) & =\frac{1}{2\left(z_{1}-z_{2}\right)^{2}}\left(\frac{z_{1} z_{2}-4 \nu}{y\left(z_{1}\right) y\left(z_{2}\right)}-1\right),
\end{aligned}
$$

причем при $z_{1}=z_{2}$ нет особенности, и при совпадаюших переменных двухточечная функция рода нуль имеет вид

$$
\begin{aligned}
& \rho^{(0 \mid 2)}(z, z)=\frac{\nu}{y^{4}(z)} \\
& M \rho^{(0 \mid 3)}\left(z_{1}, z_{2}, z_{3}\right)=\frac{2 \nu}{y\left(z_{1}\right) y\left(z_{2}\right) y\left(z_{3}\right)}\left(\frac{1}{\left(z_{1}-z_{2}\right)\left(z_{1}-z_{3}\right) y^{2}\left(z_{1}\right)}+\right. \\
& \left.\quad+\frac{1}{\left(z_{2}-z_{1}\right)\left(z_{2}-z_{3}\right) y^{2}\left(z_{2}\right)}+\frac{1}{\left(z_{3}-z_{1}\right)\left(z_{3}-z_{2}\right) y^{2}\left(z_{3}\right)}\right)= \\
& =\frac{2 \nu\left(z_{1} z_{2}+z_{2} z_{3}+z_{3} z_{1}+4 \nu\right)}{y^{3}\left(z_{1}\right) y^{3}\left(z_{2}\right) y^{3}\left(z_{3}\right)} \\
& M^{2} \rho^{(0 \mid 4)}\left(z_{1}, z_{2}, z_{3}, z_{4}\right)=\frac{\nu}{y\left(z_{1}\right)^{5} y\left(z_{2}\right)^{5} y\left(z_{3}\right)^{5} y\left(z_{4}\right)^{5} \times} \\
& \quad \times\left(2 \left[4 z_{1}^{2} z_{2}^{2} z_{3}^{2} z_{4}^{2}\left(z_{1} z_{2}+z_{1} z_{3}+z_{1} z_{4}+z_{2} z_{3}+z_{2} z_{4}+z_{3} z_{4}\right)+\right.\right. \\
& \left.+3 z_{1} z_{2} z_{3} z_{4}\left(z_{1}^{2} z_{2}^{2} z_{3}^{2}+z_{1}^{2} z_{2}^{2} z_{4}^{2}+z_{1}^{2} z_{3}^{2} z_{4}^{2}+z_{2}^{2} z_{3}^{2} z_{4}^{2}\right)\right]- \\
& -8\left[\left(z_{1}^{2} z_{2}^{2} z_{3}^{2}+z_{1}^{2} z_{2}^{2} z_{4}^{2}+z_{1}^{2} z_{3}^{2} z_{4}^{2}+z_{2}^{2} z_{3}^{2} z_{4}^{2}\right) \times\right. \\
& \times\left(z_{1} z_{2}+z_{1} z_{3}+z_{1} z_{4}+z_{2} z_{3}+z_{2} z_{4}+z_{3} z_{4}\right)+ \\
& \left.+6 z_{1} z_{2} z_{3} z_{4}\left(z_{1}^{2} z_{2}^{2}+z_{1}^{2} z_{3}^{2}+z_{1}^{2} z_{4}^{2}+z_{2}^{2} z_{3}^{2}+z_{2}^{2} z_{4}^{2}+z_{3}^{2} z_{4}^{2}\right)-6 z_{1}^{2} z_{2}^{2} z_{3}^{2} z_{4}^{2}\right] \nu- \\
& -32\left[2\left(z_{1}^{2} z_{2}^{2}+z_{1}^{2} z_{3}^{2}+z_{1}^{2} z_{4}^{2}+z_{2}^{2} z_{3}^{2}+z_{2}^{2} z_{4}^{2}+z_{3}^{2} z_{4}^{2}\right) \times\right. \\
& \times\left(z_{1} z_{2}+z_{1} z_{3}+z_{1} z_{4}+z_{2} z_{3}+z_{2} z_{4}+z_{3} z_{4}\right)+ \\
& \left.+3\left(z_{1}^{2} z_{2}^{2} z_{3}^{2}+z_{1}^{2} z_{2}^{2} z_{4}^{2}+z_{1}^{2} z_{3}^{2} z_{4}^{2}+z_{2}^{2} z_{3}^{2} z_{4}^{2}\right)-9 z_{1} z_{2} z_{3} z_{4}\left(z_{1}^{2}+z_{2}^{2}+z_{3}^{2}+z_{4}^{2}\right)\right] \nu^{2}+ \\
& +128\left[5\left(z_{1}^{2}+z_{2}^{2}+z_{3}^{2}+z_{4}^{2}\right)\left(z_{1} z_{2}+z_{1} z_{3}+z_{1} z_{4}+z_{2} z_{3}+z_{2} z_{4}+z_{3} z_{4}\right)-\right. \\
& \left.-12 z_{1} z_{2} z_{3} z_{4}\right] \nu^{3}+512\left[3\left(z_{1}^{2}+z_{2}^{2}+z_{3}^{2}+z_{4}^{2}\right)-\right. \\
& \left.\left.-8\left(z_{1} z_{2}+z_{1} z_{3}+z_{1} z_{4}+z_{2} z_{3}+z_{2} z_{4}+z_{3} z_{4}\right)\right] \nu^{4}-12288 \nu^{5}\right) .
\end{aligned}
$$

Род $p=1$ :

$$
\begin{aligned}
& M \rho^{(1 \mid 1)}(z)=\frac{\nu}{y^{5}(z)} \\
& M^{2} \rho^{(1 \mid 2)}\left(z_{1}, z_{2}\right)=\frac{\nu}{y^{7}\left(z_{1}\right) y^{7}\left(z_{2}\right)}\left(z_{1} z_{2}\left(5 z_{1}^{4}+4 z_{1}^{3} z_{2}+3 z_{1}^{2} z_{2}^{2}+4 z_{1} z_{2}^{3}+5 z_{2}^{4}\right)+\right. \\
& \quad+4 \nu\left[z_{1}^{4}-13 z_{1} z_{2}\left(z_{1}^{2}+z_{1} z_{2}+z_{2}^{2}\right)+z_{2}^{4}\right]+
\end{aligned}
$$




$$
\begin{aligned}
& \left.+16 \nu^{2}\left(-z_{1}^{2}+13 z_{1} z_{2}-z_{2}^{2}\right)+320 \nu^{3}\right), \\
& M^{3} \rho^{(1 \mid 3)}\left(z_{1}, z_{2}, z_{3}\right)=\frac{\nu}{y\left(z_{1}\right)^{9} y\left(z_{2}\right)^{9} y\left(z_{3}\right)^{9}}\left(6 \left[5 z _ { 1 } ^ { 2 } z _ { 2 } ^ { 2 } z _ { 3 } ^ { 2 } \left(z_{1}^{5} z_{2}^{5}+z_{1}^{5} z_{3}^{5}+z_{2}^{5} z_{3}^{5}+\right.\right.\right. \\
& \left.+z_{1} z_{2} z_{3}\left(z_{1}^{3} z_{2}^{4}+z_{1}^{4} z_{2}^{3}+z_{1}^{3} z_{3}^{4}+z_{1}^{4} z_{3}^{3}+z_{2}^{3} z_{3}^{4}+z_{2}^{4} z_{3}^{3}\right)\right)+ \\
& +4 z_{1}^{4} z_{2}^{4} z_{3}^{4}\left(z_{1} z_{2}^{3}+z_{1}^{3} z_{2}+z_{1} z_{3}^{3}+z_{1}^{3} z_{3}+z_{2} z_{3}^{3}+z_{2}^{3} z_{3}+z_{1}^{2} z_{2}^{2}+z_{1}^{2} z_{3}^{2}+z_{2}^{2} z_{3}^{2}\right)+ \\
& \left.+3 z_{1}^{5} z_{2}^{5} z_{3}^{5}\left(z_{1}+z_{2}+z_{3}\right)\right]+4\left[5\left(z_{1} z_{2}+z_{1} z_{3}+z_{2} z_{3}\right)\left(z_{1}^{6} z_{2}^{6}+z_{1}^{6} z_{3}^{6}+z_{2}^{6} z_{3}^{6}\right)-\right. \\
& -108 z_{1}^{2} z_{2}^{2} z_{3}^{2}\left(z_{1}^{5} z_{2}^{3}+z_{1}^{3} z_{2}^{5}+z_{1}^{5} z_{3}^{3}+z_{1}^{3} z_{3}^{5}+z_{2}^{5} z_{3}^{3}+z_{2}^{3} z_{3}^{5}+\right. \\
& \left.+z_{1} z_{2}^{2} z_{3}^{5}+z_{1} z_{2}^{5} z_{3}^{2}+z_{1}^{2} z_{2} z_{3}^{5}+z_{1}^{5} z_{2} z_{3}^{2}+z_{1}^{2} z_{2}^{5} z_{3}+z_{1}^{5} z_{2}^{2} z_{3}\right)- \\
& -96 z_{1}^{3} z_{2}^{3} z_{3}^{3}\left(z_{1} z_{2} z_{3}^{3}+z_{1} z_{2}^{3} z_{3}+z_{1}^{3} z_{2} z_{3}+z_{1}^{2} z_{2}^{3}+z_{1}^{3} z_{2}^{2}+\right. \\
& \left.+z_{1}^{2} z_{3}^{3}+z_{1}^{3} z_{3}^{2}+z_{2}^{2} z_{3}^{3}+z_{2}^{3} z_{3}^{2}\right)-180 z_{1}^{4} z_{2}^{4} z_{3}^{4}\left(z_{1} z_{2}+z_{1} z_{3}+z_{2} z_{3}\right)+ \\
& \left.+12 z_{1}^{2} z_{2}^{2} z_{3}^{2}\left(z_{1}^{4} z_{2}^{4}+z_{1}^{4} z_{3}^{4}+z_{2}^{4} z_{3}^{4}\right)\right] \nu+16\left[-\left(z_{1}^{6} z_{2}^{6}+z_{1}^{6} z_{3}^{6}+z_{2}^{6} z_{3}^{6}\right)-\right. \\
& -21\left(z_{1}^{5} z_{2}^{5}\left(z_{1}^{2}+z_{2}^{2}\right)+z_{1}^{5} z_{3}^{5}\left(z_{1}^{2}+z_{3}^{2}\right)+z_{2}^{5} z_{3}^{5}\left(z_{2}^{2}+z_{3}^{2}\right)+\right. \\
& \left.+z_{1} z_{2} z_{3}\left(z_{1}^{3} z_{2}^{6}+z_{1}^{6} z_{2}^{3}+z_{1}^{3} z_{3}^{6}+z_{1}^{6} z_{3}^{3}+z_{2}^{3} z_{3}^{6}+z_{2}^{6} z_{3}^{3}\right)\right)- \\
& -27 z_{1} z_{2} z_{3}\left(z_{1}^{4} z_{2}^{5}+z_{1}^{5} z_{2}^{4}+z_{1}^{4} z_{3}^{5}+z_{1}^{5} z_{3}^{4}+z_{2}^{4} z_{3}^{5}+z_{2}^{5} z_{3}^{4}\right)- \\
& -30 z_{1}^{2} z_{2}^{2} z_{3}^{2}\left(z_{1}^{4} z_{2}^{2}+z_{1}^{2} z_{2}^{4}+z_{1}^{4} z_{3}^{2}+z_{1}^{2} z_{3}^{4}+z_{2}^{4} z_{3}^{2}+z_{2}^{2} z_{3}^{4}\right)+ \\
& +132 z_{1}^{3} z_{2}^{3} z_{3}^{3}\left(z_{1}^{3}+z_{2}^{3}+z_{3}^{3}\right)+ \\
& +156 z_{1}^{2} z_{2}^{2} z_{3}^{2}\left(z_{1} z_{2}^{5}+z_{1}^{5} z_{2}+z_{1} z_{3}^{5}+z_{1}^{5} z_{3}+z_{2} z_{3}^{5}+z_{2}^{5} z_{3}\right)+ \\
& +378 z_{1}^{3} z_{2}^{3} z_{3}^{3}\left(z_{1} z_{2}^{2}+z_{1}^{2} z_{2}+z_{1} z_{3}^{2}+z_{1}^{2} z_{3}+z_{2} z_{3}^{2}+z_{2}^{2} z_{3}\right)+ \\
& \left.+396 z_{1}^{2} z_{2}^{2} z_{3}^{2}\left(z_{1}^{3} z_{2}^{3}+z_{1}^{3} z_{3}^{3}+z_{2}^{3} z_{3}^{3}\right)+234 z_{1}^{4} z_{2}^{4} z_{3}^{4}\right] \nu^{2}+ \\
& +192\left[-\left(z_{1}^{4} z_{2}^{4}\left(z_{1}^{2}+z_{2}^{2}\right)+z_{1}^{4} z_{3}^{4}\left(z_{1}^{2}+z_{3}^{2}\right)+z_{2}^{4} z_{3}^{4}\left(z_{2}^{2}+z_{3}^{2}\right)\right)+\right. \\
& +60 z_{1}^{2} z_{2}^{2} z_{3}^{2}\left(z_{1}^{2} z_{2}^{2}+z_{1}^{2} z_{3}^{2}+z_{2}^{2} z_{3}^{2}\right)+ \\
& +9\left(z_{1}^{3} z_{2}^{3}\left(z_{1}^{4}+z_{2}^{4}\right)+z_{1}^{3} z_{3}^{3}\left(z_{1}^{4}+z_{3}^{4}\right)+z_{2}^{3} z_{3}^{3}\left(z_{2}^{4}+z_{3}^{4}\right)+\right. \\
& \left.+z_{1} z_{2} z_{3}\left(z_{1} z_{2}^{6}+z_{1}^{6} z_{2}+z_{1} z_{3}^{6}+z_{1}^{6} z_{3}+z_{2} z_{3}^{6}+z_{2}^{6} z_{3}\right)\right)+27\left(z_{1}^{5} z_{2}^{5}+z_{1}^{5} z_{3}^{5}+z_{2}^{5} z_{3}^{5}\right)- \\
& -156 z_{1}^{2} z_{2}^{2} z_{3}^{2}\left(z_{1} z_{2}^{3}+z_{1}^{3} z_{2}+z_{1} z_{3}^{3}+z_{1}^{3} z_{3}+z_{2} z_{3}^{3}+z_{2}^{3} z_{3}\right)+ \\
& +48 z_{1}^{2} z_{2}^{2} z_{3}^{2}\left(z_{1}^{4}+z_{2}^{4}+z_{3}^{4}\right)+ \\
& +13 z_{1} z_{2} z_{3}\left(z_{1}^{2} z_{2}^{5}+z_{1}^{5} z_{2}^{2}+z_{1}^{2} z_{3}^{5}+z_{1}^{5} z_{3}^{2}+z_{2}^{2} z_{3}^{5}+z_{2}^{5} z_{3}^{2}\right)- \\
& -144 z_{1}^{3} z_{2}^{3} z_{3}^{3}\left(z_{1}+z_{2}+z_{3}\right)+ \\
& \left.+31 z_{1} z_{2} z_{3}\left(z_{1}^{4} z_{2}^{3}+z_{1}^{3} z_{2}^{4}+z_{1}^{4} z_{3}^{3}+z_{1}^{3} z_{3}^{4}+z_{2}^{4} z_{3}^{3}+z_{2}^{3} z_{3}^{4}\right)\right] \nu^{3}- \\
& -256\left[3\left(z_{1}^{4} z_{2}^{4}+z_{1}^{4} z_{3}^{4}+z_{2}^{4} z_{3}^{4}\right)+\right. \\
& +6\left(z_{1}^{2} z_{2}^{2}\left(z_{1}^{4}+z_{2}^{4}\right)+z_{1}^{2} z_{3}^{2}\left(z_{1}^{4}+z_{3}^{4}\right)+z_{2}^{2} z_{3}^{2}\left(z_{2}^{4}+z_{3}^{4}\right)\right)+ \\
& +129\left(z_{1}^{3} z_{2}^{3}\left(z_{1}^{2}+z_{2}^{2}\right)+z_{1}^{3} z_{3}^{3}\left(z_{1}^{2}+z_{3}^{2}\right)+z_{2}^{3} z_{3}^{3}\left(z_{2}^{2}+z_{3}^{2}\right)\right)+ \\
& +52 z_{1} z_{2} z_{3}\left(z_{1}^{5}+z_{2}^{5}+z_{3}^{5}\right)+46\left(z_{1} z_{2}\left(z_{1}^{6}+z_{2}^{6}\right)+z_{1} z_{3}\left(z_{1}^{6}+z_{3}^{6}\right)+z_{2} z_{3}\left(z_{2}^{6}+z_{3}^{6}\right)\right)+ \\
& +378 z_{1}^{2} z_{2}^{2} z_{3}^{2}\left(z_{1} z_{2}+z_{1} z_{3}+z_{2} z_{3}\right)+ \\
& +153 z_{1} z_{2} z_{3}\left(z_{1}^{2} z_{2}^{3}+z_{1}^{3} z_{2}^{2}+z_{1}^{2} z_{3}^{3}+z_{1}^{3} z_{3}^{2}+z_{2}^{2} z_{3}^{3}+z_{2}^{3} z_{3}^{2}\right)+ \\
& +540 z_{1}^{2} z_{2}^{2} z_{3}^{2}\left(z_{1}^{2}+z_{2}^{2}+z_{3}^{2}\right)+
\end{aligned}
$$




$$
\begin{aligned}
& \left.+135 z_{1} z_{2} z_{3}\left(z_{1} z_{2}^{4}+z_{1}^{4} z_{2}+z_{1} z_{3}^{4}+z_{1}^{4} z_{3}+z_{2} z_{3}^{4}+z_{2}^{4} z_{3}\right)\right] \nu^{4}+ \\
& +1024\left[-10\left(z_{1}^{6}+z_{2}^{6}+z_{3}^{6}\right)+75\left(z_{1}^{2} z_{2}^{2}\left(z_{1}^{2}+z_{2}^{2}\right)+\right.\right. \\
& \left.+z_{1}^{2} z_{3}^{2}\left(z_{1}^{2}+z_{3}^{2}\right)+z_{2}^{2} z_{3}^{2}\left(z_{2}^{2}+z_{3}^{2}\right)\right)+ \\
& +174\left(z_{1} z_{2}\left(z_{1}^{4}+z_{2}^{4}\right)+z_{1} z_{3}\left(z_{1}^{4}+z_{3}^{4}\right)+z_{2} z_{3}\left(z_{2}^{4}+z_{3}^{4}\right)\right)+ \\
& +237\left(z_{1}^{3} z_{2}^{3}+z_{1}^{3} z_{3}^{3}+z_{2}^{3} z_{3}^{3}\right)+249 z_{1} z_{2} z_{3}\left(z_{1}^{2} z_{2}+z_{1} z_{2}^{2}+z_{1}^{2} z_{3}+\right. \\
& \left.\left.+z_{1} z_{3}^{2}+z_{2}^{2} z_{3}+z_{2} z_{3}^{2}\right)+186 z_{1} z_{2} z_{3}\left(z_{1}^{3}+z_{2}^{3}+z_{3}^{3}\right)+828 z_{1}^{2} z_{2}^{2} z_{3}^{2}\right] \nu^{5}- \\
& -12288\left[80\left(z_{1} z_{2}\left(z_{1}^{2}+z_{2}^{2}\right)+z_{1} z_{3}\left(z_{1}^{2}+z_{3}^{2}\right)+z_{2} z_{3}\left(z_{2}^{2}+z_{3}^{2}\right)\right)-12\left(z_{1}^{4}+z_{2}^{4}+z_{3}^{4}\right)+\right. \\
& \left.+39\left(z_{1}^{2} z_{2}^{2}+z_{1}^{2} z_{3}^{2}+z_{2}^{2} z_{3}^{2}\right)+82 z_{1} z_{2} z_{3}\left(z_{1}+z_{2}+z_{3}\right)\right] \nu^{6}+ \\
& \left.+344064\left[-2\left(z_{1}^{2}+z_{2}^{2}+z_{3}^{2}\right)+7\left(z_{1} z_{2}+z_{1} z_{3}+z_{2} z_{3}\right)\right] \nu^{7}+3342336 \nu^{8}\right) .
\end{aligned}
$$

Род $p=2$ :

$$
\begin{aligned}
& M^{3} \rho^{(2 \mid 1)}(z)=\frac{21 \nu\left(z^{2}+\nu\right)}{y^{11}(z)}, \\
& M^{4} \rho^{(2 \mid 2)}\left(z_{1}, z_{2}\right)=\frac{\nu}{y\left(z_{1}\right)^{13} y\left(z_{2}\right)^{13}}\left(189 z_{1}^{11} z_{2}^{3}+168 z_{1}^{10} z_{2}^{4}+147 z_{1}^{9} z_{2}^{5}+156 z_{1}^{8} z_{2}^{6}+\right. \\
& \quad+165 z_{1}^{7} z_{2}^{7}+156 z_{1}^{6} z_{2}^{8}+147 z_{1}^{5} z_{2}^{9}+168 z_{1}^{4} z_{2}^{10}+189 z_{1}^{3} z_{2}^{11}+ \\
& \quad+\left[399 z_{1}^{11} z_{2}+462 z_{1}^{10} z_{2}^{2}-4011 z_{1}^{9} z_{2}^{3}-3664 z_{1}^{8} z_{2}^{4}-3317 z_{1}^{7} z_{2}^{5}-3342 z_{1}^{6} z_{2}^{6}-\right. \\
& \left.\quad-3317 z_{1}^{5} z_{2}^{7}-3664 z_{1}^{4} z_{2}^{8}-4011 z_{1}^{3} z_{2}^{9}+462 z_{1}^{2} z_{2}^{10}+399 z_{1} z_{2}^{11}\right] \nu+ \\
& \quad+\left[84 z_{1}^{10}-9408 z_{1}^{9} z_{2}-10516 z_{1}^{8} z_{2}^{2}+33736 z_{1}^{7} z_{2}^{3}+30980 z_{1}^{6} z_{2}^{4}+27624 z_{1}^{5} z_{2}^{5}+\right. \\
& \left.\quad+30980 z_{1}^{4} z_{2}^{6}+33736 z_{1}^{3} z_{2}^{7}-10516 z_{1}^{2} z_{2}^{8}-9408 z_{1} z_{2}^{9}+84 z_{2}^{10}\right] \nu^{2}+ \\
& \quad+\left[-1696 z_{1}^{8}+92368 z_{1}^{7} z_{2}+101120 z_{1}^{6} z_{2}^{2}-129648 z_{1}^{5} z_{2}^{3}-133248 z_{1}^{4} z_{2}^{4}-\right. \\
& \left.\quad-129648 z_{1}^{3} z_{2}^{5}+101120 z_{1}^{2} z_{2}^{6}+92368 z_{1} z_{2}^{7}-1696 z_{2}^{8}\right] \nu^{3}+ \\
& \quad+\left[12992 z_{1}^{6}-487936 z_{1}^{5} z_{2}-491072 z_{1}^{4} z_{2}^{2}+202752 z_{1}^{3} z_{2}^{3}-\right. \\
& \left.\quad-491072 z_{1}^{2} z_{2}^{4}-487936 z_{1} z_{2}^{5}+12992 z_{2}^{6}\right] \nu^{4}+ \\
& \quad+\left[-77312 z_{1}^{4}+1415936 z_{1}^{3} z_{2}+2519552 z_{1}^{2} z_{2}^{2}+1415936 z_{1} z_{2}^{3}-77312 z_{2}^{4}\right] \nu^{5}+ \\
& \left.\quad+\left[-560128 z_{1}^{2}-2951168 z_{1} z_{2}-560128 z_{2}^{2}\right] \nu^{6}-1736704 \nu^{7}\right) .
\end{aligned}
$$

Род $p=3$ :

$$
M^{5} \rho^{(3 \mid 1)}(z)=\frac{11 \nu\left(135 z_{1}^{4}+558 z_{1}^{2} \nu+158 \nu^{2}\right)}{y(z)^{17}} .
$$

Отметим, что все эти полиплотности определены для любых конечных значений $N$ и не имеют отношения к пределу $N \rightarrow \infty$. Альтернативный подход к этому вопросу изучался в работе [53].

1.5. Первые гауссовы полиплотности: первые члены $N$-разложений. В этом пункте мы положим $M=1$ и $g=1$, так что $\nu=N$. 
Одноточечные функции:

$$
\begin{aligned}
\rho_{G}^{(0 \mid 1)}(x)= & \frac{1}{x} N+\frac{1}{x^{3}} N^{2}+\frac{2}{x^{5}} N^{3}+\frac{5}{x^{7}} N^{4}+\frac{14}{x^{9}} N^{5}+\frac{42}{x^{11}} N^{6}+ \\
& +\frac{132}{x^{13}} N^{7}+\frac{429}{x^{15}} N^{8}+\frac{1430}{x^{17}} N^{9}+O\left(N^{10}\right), \\
\rho_{G}^{(1 \mid 1)}(x)= & \frac{1}{x^{5}} N+\frac{10}{x^{7}} N^{2}+\frac{70}{x^{9}} N^{3}+\frac{420}{x^{11}} N^{4}+\frac{2310}{x^{13}} N^{5}+ \\
& +\frac{12012}{x^{15}} N^{6}+\frac{60060}{x^{17}} N^{7}+\frac{291720}{x^{19}} N^{8}+\frac{1385670}{x^{21}} N^{9}+O\left(N^{10}\right), \\
\rho_{G}^{(2 \mid 1)}(x)= & \frac{21}{x^{9}} N+\frac{483}{x^{11}} N^{2}+\frac{6468}{x^{13}} N^{3}+\frac{66066}{x^{15}} N^{4}+\frac{570570}{x^{17}} N^{5}+ \\
& +\frac{4390386}{x^{19}} N^{6}+\frac{31039008}{x^{21}} N^{7}+\frac{205633428}{x^{23}} N^{8}+\frac{1293938646}{x^{25}} N^{9}+O\left(N^{10}\right), \\
\rho_{G}^{(3 \mid 1)}(x)= & \frac{1485}{x^{13}} N+\frac{56628}{x^{15}} N^{2}+\frac{1169740}{x^{17}} N^{3}+\frac{17454580}{x^{19}} N^{4}+ \\
& +\frac{211083730}{x^{21}} N^{5}+\frac{2198596400}{x^{23}} N^{6}+\frac{20465052608}{x^{25}} N^{7}+ \\
& +\frac{174437377400}{x^{27}} N^{8}+\frac{1384928666550}{x^{29}} N^{9}+O\left(N^{10}\right) .
\end{aligned}
$$

Двухточечные функции:

$$
\begin{aligned}
& \rho_{G}^{(0 \mid 2)}(x, y)= \frac{1}{2(x-y)^{2}}\left[-1+\left(1-\frac{4 N}{x y}\right)\left(1+\frac{2 N}{x^{2}}+\frac{6 N^{2}}{x^{4}}+\right.\right. \\
&\left.+\frac{20 N^{3}}{x^{6}}+\frac{70 N^{4}}{x^{8}}+\frac{252 N^{5}}{x^{10}}+\cdots\right) \times \\
&\left.\times\left(1+\frac{2 N}{y^{2}}+\frac{6 N^{2}}{y^{4}}+\frac{20 N^{3}}{y^{6}}+\frac{70 N^{4}}{y^{8}}+\frac{252 N^{5}}{y^{10}}+\cdots\right)\right]= \\
&= \frac{N}{x^{2} y^{2}}+N^{2}\left(\frac{3}{x^{4} y^{2}}+\frac{2}{x^{3} y^{3}}+\frac{3}{x^{2} y^{4}}\right)+ \\
&+N^{3}\left(\frac{10}{x^{6} y^{2}}+\frac{8}{x^{5} y^{3}}+\frac{12}{x^{4} y^{4}}+\frac{8}{x^{3} y^{5}}+\frac{10}{x^{2} y^{6}}\right)+ \\
&+N^{4}\left(\frac{35}{x^{8} y^{2}}+\frac{30}{x^{7} y^{3}}+\frac{45}{x^{6} y^{4}}+\frac{36}{x^{5} y^{5}}+\frac{45}{x^{4} y^{6}}+\frac{30}{x^{3} y^{7}}+\frac{35}{x^{2} y^{8}}\right)+ \\
&+N^{5}\left(\frac{126}{x^{10} y^{2}}+\frac{112}{x^{9} y^{3}}+\frac{168}{x^{8} y^{4}}+\frac{144}{x^{7} y^{5}}+\frac{180}{x^{6} y^{6}}+\frac{144}{x^{5} y^{7}}+\right. \\
&\left.+\frac{168}{x^{4} y^{8}}+\frac{112}{x^{3} y^{9}}+\frac{126}{x^{2} y^{10}}\right)+O\left(N^{6}\right), \\
&\left(\frac{5}{x^{6} y^{2}}+\frac{4}{x^{5} y^{3}}+\frac{3}{x^{4} y^{4}}+\frac{4}{x^{3} y^{5}}+\frac{5}{x^{2} y^{6}}\right) N^{2}+ \\
&+\left(\frac{70}{x^{8} y^{2}}+\frac{60}{x^{7} y^{3}}+\frac{60}{x^{6} y^{4}}+\frac{60}{x^{5} y^{5}}+\frac{60}{x^{4} y^{6}}+\frac{60}{x^{3} y^{7}}+\frac{70}{x^{2} y^{8}}\right) N^{2}+ \\
& \rho_{G}^{(1 \mid 2)(x, y)}+\left(\frac{630}{x^{10} y^{2}}+\frac{560}{x^{9} y^{3}}+\frac{630}{x^{8} y^{4}}+\frac{600}{x^{7} y^{5}}+\frac{600}{x^{6} y^{6}}+\right. \\
& \\
&+(x)
\end{aligned}
$$




$$
\begin{aligned}
+ & \left.\frac{600}{x^{5} y^{7}}+\frac{630}{x^{4} y^{8}}+\frac{560}{x^{3} y^{9}}+\frac{630}{x^{2} y^{10}}\right) N^{3}+ \\
& +\left(\frac{4620}{x^{12} y^{2}}+\frac{4200}{x^{11} y^{3}}+\frac{5040}{x^{10} y^{4}}+\frac{4760}{x^{9} y^{5}}+\frac{4900}{x^{8} y^{6}}+\frac{4800}{x^{7} y^{7}}+\right. \\
& \left.+\frac{4900}{x^{6} y^{8}}+\frac{4760}{x^{5} y^{9}}+\frac{5040}{x^{4} y^{10}}+\frac{4200}{x^{3} y^{11}}+\frac{4620}{x^{2} y^{12}}\right) N^{4}+ \\
& +\left(\frac{30030}{x^{14} y^{2}}+\frac{27720}{x^{13} y^{3}}+\frac{34650}{x^{12} y^{4}}+\frac{32760}{x^{11} y^{5}}+\frac{34650}{x^{10} y^{6}}+\frac{33600}{x^{9} y^{7}}+\frac{34300}{y^{8} x^{8}}+\right. \\
& \left.+\frac{33600}{x^{7} y^{9}}+\frac{34650}{x^{6} y^{10}}+\frac{32760}{x^{5} y^{11}}+\frac{34650}{x^{4} y^{12}}+\frac{27720}{x^{3} y^{13}}+\frac{30030}{x^{2} y^{14}}\right) N^{5}+O\left(N^{6}\right), \\
\rho_{G}^{(2 \mid 2)}(x, y)= & \left.\frac{189}{x y^{9}}+\frac{168}{x^{8} y^{2}}+\frac{147}{x^{7} y^{3}}+\frac{156}{x^{6} y^{4}}+\frac{165}{x^{5} y^{5}}+\frac{156}{x^{4} y^{6}}+\frac{147}{x^{3} y^{7}}+\frac{168}{x^{2} y^{8}}+\frac{189}{x^{9} y}\right) N+ \\
& +\left(\frac{5313}{x^{11} y}+\frac{4830}{x^{10} y^{2}}+\frac{4725}{x^{9} y^{3}}+\frac{4760}{x^{8} y^{4}}+\frac{4795}{x^{7} y^{5}}+\frac{4770}{x^{6} y^{6}}+\right. \\
& \left.+\frac{4795}{x^{5} y^{7}}+\frac{4760}{x^{4} y^{8}}+\frac{4725}{x^{3} y^{9}}+\frac{4830}{x^{2} y^{10}}+\frac{5313}{x y^{11}}\right) N^{2}+ \\
& +\left(\frac{84084}{x^{13} y}+\frac{77616}{x^{12} y^{2}}+\frac{81774}{x^{11} y^{3}}+\frac{80640}{x^{10} y^{4}}+\frac{80640}{x^{9} y^{5}}+\frac{80640}{x^{8} y^{6}}+\frac{81340}{x^{7} y^{7}}+\right. \\
& \left.+\frac{80640}{x^{6} y^{8}}+\frac{80640}{x^{5} y^{9}}+\frac{80640}{x^{4} y^{10}}+\frac{81774}{x^{3} y^{11}}+\frac{77616}{x^{2} y^{12}}+\frac{84084}{x y^{13}}\right) N^{3}+O\left(N^{4}\right) .
\end{aligned}
$$

Трехточечные функции:

$$
\begin{aligned}
\rho_{G}^{(0 \mid 3)}(x, y, z)= & 2\left(\frac{1}{x^{2} y^{2} z^{3}}+\frac{1}{x^{2} y^{3} z^{2}}+\frac{1}{x^{3} y^{2} z^{2}}\right) N+ \\
& +\left[1 2 \left(\frac{1}{x^{2} y^{2} z^{5}}+\frac{1}{x^{2} y^{5} z^{2}}+\frac{1}{x^{5} y^{2} z^{2}}+\frac{1}{x^{2} y^{3} z^{4}}+\frac{1}{x^{3} y^{4} z^{2}}+\frac{1}{x^{4} y^{3} z^{2}}+\right.\right. \\
& \left.\left.+\frac{1}{x^{2} y^{4} z^{3}}+\frac{1}{x^{3} y^{2} z^{4}}+\frac{1}{x^{4} y^{2} z^{3}}\right)+\frac{8}{x^{3} y^{3} z^{3}}\right] N^{2}+ \\
& +\left[6 0 \left(\frac{1}{x^{3} y^{6} z^{2}}+\frac{1}{x^{6} y^{3} z^{2}}+\frac{1}{z^{6} x^{3} y^{2}}+\frac{1}{z^{3} x^{2} y^{6}}+\frac{1}{z^{3} x^{6} y^{2}}+\frac{1}{z^{6} x^{2} y^{3}}+\right.\right. \\
& \left.+\frac{1}{x^{7} y^{2} z^{2}}+\frac{1}{z^{7} x^{2} y^{2}}+\frac{1}{x^{2} y^{7} z^{2}}\right)+72\left(\frac{1}{z^{3} x^{4} y^{4}}+\frac{1}{z^{4} x^{4} y^{3}}+\frac{1}{z^{4} x^{3} y^{4}}+\right. \\
& \left.+\frac{1}{x^{5} y^{4} z^{2}}+\frac{1}{z^{5} x^{4} y^{2}}+\frac{1}{z^{4} x^{5} y^{2}}+\frac{1}{z^{4} x^{2} y^{5}}+\frac{1}{z^{5} x^{2} y^{4}}+\frac{1}{x^{4} y^{5} z^{2}}\right)+ \\
& \left.+48\left(\frac{1}{z^{3} x^{3} y^{5}}+\frac{1}{z^{5} x^{3} y^{3}}+\frac{1}{z^{3} x^{5} y^{3}}\right)\right] N^{3}+ \\
& +\left[2 8 0 \left(\frac{1}{z^{9} x^{2} y^{2}}+\frac{1}{x^{9} y^{2} z^{2}}+\frac{1}{x^{2} y^{9} z^{2}}+\frac{1}{z^{3} x^{8} y^{2}}+\frac{1}{z^{8} x^{3} y^{2}}+\right.\right. \\
& \left.+\frac{1}{z^{3} x^{2} y^{8}}+\frac{1}{z^{8} x^{2} y^{3}}+\frac{1}{x^{8} y^{3} z^{2}}+\frac{1}{x^{3} y^{8} z^{2}}\right)+ \\
& +360\left(\frac{1}{z^{4} x^{7} y^{2}}+\frac{1}{z^{7} x^{4} y^{2}}+\frac{1}{z^{4} x^{2} y^{7}}+\frac{1}{z^{7} x^{2} y^{4}}+\frac{1}{x^{7} y^{4} z^{2}}+\frac{1}{x^{4} y^{7} z^{2}}+\right.
\end{aligned}
$$




$$
\begin{aligned}
& +\frac{1}{z^{5} x^{6} y^{2}}+\frac{1}{z^{6} x^{5} y^{2}}+\frac{1}{z^{5} x^{2} y^{6}}+\frac{1}{z^{6} x^{2} y^{5}}+\frac{1}{x^{5} y^{6} z^{2}}+\frac{1}{x^{6} y^{5} z^{2}}+ \\
& \left.+\frac{1}{z^{4} x^{6} y^{3}}+\frac{1}{z^{3} x^{6} y^{4}}+\frac{1}{z^{6} x^{4} y^{3}}+\frac{1}{z^{3} x^{4} y^{6}}+\frac{1}{z^{6} x^{3} y^{4}}+\frac{1}{z^{4} x^{3} y^{6}}\right)+ \\
& +240\left(\frac{1}{z^{3} x^{7} y^{3}}+\frac{1}{z^{3} x^{3} y^{7}}+\frac{1}{z^{7} x^{3} y^{3}}\right)+ \\
& +288\left(\frac{1}{z^{3} x^{5} y^{5}}+\frac{1}{z^{5} x^{3} y^{5}}+\frac{1}{z^{5} x^{5} y^{3}}\right)+ \\
& \left.+432\left(\frac{1}{z^{4} x^{5} y^{4}}+\frac{1}{z^{4} x^{4} y^{5}}+\frac{1}{z^{5} x^{4} y^{4}}\right)\right] N^{4}+ \\
& +\left[1 6 8 0 \left(\frac{1}{z^{3} x^{8} y^{4}}+\frac{1}{z^{4} x^{8} y^{3}}+\frac{1}{z^{4} x^{3} y^{8}}+\frac{1}{z^{8} x^{3} y^{4}}+\frac{1}{z^{8} x^{4} y^{3}}+\frac{1}{z^{3} x^{4} y^{8}}+\right.\right. \\
& +\frac{1}{x^{4} y^{9} z^{2}}+\frac{1}{z^{9} x^{4} y^{2}}+\frac{1}{z^{4} x^{2} y^{9}}+\frac{1}{z^{9} x^{2} y^{4}}+\frac{1}{x^{9} y^{4} z^{2}}+\frac{1}{z^{4} x^{9} y^{2}}+ \\
& \left.+\frac{1}{z^{8} x^{5} y^{2}}+\frac{1}{z^{5} x^{2} y^{8}}+\frac{1}{z^{5} x^{8} y^{2}}+\frac{1}{z^{8} x^{2} y^{5}}+\frac{1}{x^{8} y^{5} z^{2}}+\frac{1}{x^{5} y^{8} z^{2}}\right)+ \\
& +1440\left(\frac{1}{z^{3} x^{7} y^{5}}+\frac{1}{z^{5} x^{7} y^{3}}+\frac{1}{z^{5} x^{3} y^{7}}+\frac{1}{z^{7} x^{3} y^{5}}+\frac{1}{z^{3} x^{5} y^{7}}+\frac{1}{z^{7} x^{5} y^{3}}\right)+ \\
& +2160\left(\frac{1}{z^{4} x^{5} y^{6}}+\frac{1}{z^{6} x^{5} y^{4}}+\frac{1}{z^{5} x^{6} y^{4}}+\frac{1}{z^{6} x^{4} y^{5}}+\frac{1}{z^{4} x^{6} y^{5}}+\right. \\
& \left.+\frac{1}{z^{5} x^{4} y^{6}}+\frac{1}{z^{4} x^{7} y^{4}}+\frac{1}{z^{4} x^{4} y^{7}}+\frac{1}{z^{7} x^{4} y^{4}}\right)+ \\
& +1120\left(\frac{1}{z^{3} x^{3} y^{9}}+\frac{1}{z^{9} x^{3} y^{3}}+\frac{1}{z^{3} x^{9} y^{3}}\right)+ \\
& +1800\left(\frac{1}{z^{7} x^{6} y^{2}}+\frac{1}{z^{6} x^{7} y^{2}}+\frac{1}{x^{7} y^{6} z^{2}}+\frac{1}{z^{7} x^{2} y^{6}}+\frac{1}{z^{6} x^{2} y^{7}}+\frac{1}{x^{6} y^{7} z^{2}}+\right. \\
& \left.+\frac{1}{z^{6} x^{3} y^{6}}+\frac{1}{z^{3} x^{6} y^{6}}+\frac{1}{z^{6} x^{6} y^{3}}\right)+1260\left(\frac{1}{z^{11} x^{2} y^{2}}+\frac{1}{x^{11} y^{2} z^{2}}+\right. \\
& +\frac{1}{x^{2} y^{11} z^{2}}+\frac{1}{z^{3} x^{10} y^{2}}+\frac{1}{z^{10} x^{3} y^{2}}+\frac{1}{z^{3} x^{2} y^{10}}+ \\
& \left.\left.+\frac{1}{z^{10} x^{2} y^{3}}+\frac{1}{x^{10} y^{3} z^{2}}+\frac{1}{x^{3} y^{10} z^{2}}\right)+\frac{1728}{z^{5} x^{5} y^{5}}\right] N^{5}+O\left(N^{6}\right), \\
& \rho_{G}^{(1 \mid 3)}(x, y, z)=\left[2 4 \left(\frac{1}{x^{3} z y^{4}}+\frac{1}{x^{4} z y^{3}}+\frac{1}{z^{4} x y^{3}}+\frac{1}{z^{3} x^{4} y}+\frac{1}{z^{4} x^{3} y}+\frac{1}{z^{3} x y^{4}}+\right.\right. \\
& \left.+\frac{1}{z^{2} x^{2} y^{4}}+\frac{1}{z^{2} x^{4} y^{2}}+\frac{1}{z^{4} x^{2} y^{2}}\right)+18\left(\frac{1}{z^{2} x^{3} y^{3}}+\frac{1}{z^{3} x^{2} y^{3}}+\frac{1}{z^{3} x^{3} y^{2}}\right)+ \\
& +30\left(\frac{1}{x^{2} z y^{5}}+\frac{1}{z^{5} x^{2} y}+\frac{1}{z^{2} x y^{5}}+\frac{1}{z^{5} x y^{2}}+\frac{1}{z^{2} x^{5} y}+\right. \\
& \left.\left.+\frac{1}{x^{5} z y^{2}}+\frac{1}{x^{6} z y}+\frac{1}{x z y^{6}}+\frac{1}{z^{6} x y}\right)\right] N+
\end{aligned}
$$




$$
\begin{aligned}
& +\left[540\left(\frac{1}{x^{5} z y^{4}}+\frac{1}{z^{4} x^{5} y}+\frac{1}{z^{5} x^{4} y}+\frac{1}{z^{5} x y^{4}}+\frac{1}{x^{4} z y^{5}}+\frac{1}{z^{4} x y^{5}}\right)+\right. \\
& +480\left(\frac{1}{z^{2} x^{5} y^{3}}+\frac{1}{z^{3} x^{5} y^{2}}+\frac{1}{z^{5} x^{3} y^{2}}+\frac{1}{z^{5} x^{2} y^{3}}+\frac{1}{z^{3} x^{2} y^{5}}+\frac{1}{z^{2} x^{3} y^{5}}\right)+ \\
& +540\left(\frac{1}{z^{6} x^{3} y}+\frac{1}{x^{3} z y^{6}}+\frac{1}{z^{3} x y^{6}}+\frac{1}{x^{6} z y^{3}}+\frac{1}{z^{3} x^{6} y}+\frac{1}{z^{6} x y^{3}}\right)+ \\
& +560\left(\frac{1}{z^{7} x y^{2}}+\frac{1}{x^{2} z y^{7}}+\frac{1}{z^{2} x y^{7}}+\frac{1}{z^{2} x^{7} y}+\frac{1}{z^{7} x^{2} y}+\frac{1}{x^{7} z y^{2}}\right)+ \\
& +480\left(\frac{1}{z^{4} x^{2} y^{4}}+\frac{1}{z^{4} x^{4} y^{2}}+\frac{1}{z^{2} x^{4} y^{4}}\right)+560\left(\frac{1}{z^{8} x y}+\frac{1}{x z y^{8}}+\frac{1}{x^{8} z y}\right)+ \\
& +468\left(\frac{1}{z^{4} x^{3} y^{3}}+\frac{1}{z^{3} x^{3} y^{4}}+\frac{1}{z^{3} x^{4} y^{3}}\right)+ \\
& \left.+480\left(\frac{1}{z^{2} x^{2} y^{6}}+\frac{1}{z^{6} x^{2} y^{2}}+\frac{1}{z^{2} x^{6} y^{2}}\right)\right] N^{2}+ \\
& +\left[6 3 0 0 \left(\frac{1}{z^{3} x^{7} y^{2}}+\frac{1}{z^{7} x^{3} y^{2}}+\frac{1}{z^{2} x^{7} y^{3}}+\frac{1}{z^{7} x^{2} y^{3}}+\right.\right. \\
& \left.+\frac{1}{z^{3} x^{2} y^{7}}+\frac{1}{z^{2} x^{3} y^{7}}+\frac{1}{x^{10} z y}+\frac{1}{z^{10} x y}+\frac{1}{x z y^{10}}\right)+ \\
& +6000\left(\frac{1}{z^{4} x^{2} y^{6}}+\frac{1}{z^{2} x^{6} y^{4}}+\frac{1}{z^{6} x^{2} y^{4}}+\frac{1}{z^{4} x^{6} y^{2}}+\right. \\
& \left.+\frac{1}{z^{2} x^{4} y^{6}}+\frac{1}{z^{6} x^{4} y^{2}}+\frac{1}{z^{2} x^{5} y^{5}}+\frac{1}{z^{5} x^{2} y^{5}}+\frac{1}{z^{5} x^{5} y^{2}}\right)+ \\
& +6300\left(\frac{1}{z^{2} x^{9} y}+\frac{1}{x^{9} z y^{2}}+\frac{1}{x^{2} z y^{9}}+\frac{1}{z^{9} x y^{2}}+\frac{1}{z^{2} x y^{9}}+\frac{1}{z^{9} x^{2} y}\right)+ \\
& +6660\left(\frac{1}{z^{6} x^{3} y^{3}}+\frac{1}{z^{3} x^{3} y^{6}}+\frac{1}{z^{3} x^{6} y^{3}}\right)+ \\
& +6720\left(\frac{1}{z^{4} x^{7} y}+\frac{1}{x^{7} z y^{4}}+\frac{1}{z^{7} x y^{4}}+\frac{1}{z^{4} x y^{7}}+\frac{1}{z^{7} x^{4} y}+\frac{1}{x^{4} z y^{7}}\right)+ \\
& +5600\left(\frac{1}{z^{8} x^{2} y^{2}}+\frac{1}{z^{2} x^{2} y^{8}}+\frac{1}{z^{2} x^{8} y^{2}}\right)+ \\
& +6300\left(\frac{1}{z^{2} x^{9} y}+\frac{1}{x^{9} z y^{2}}+\frac{1}{x^{2} z y^{9}}+\frac{1}{z^{9} x y^{2}}+\frac{1}{z^{2} x y^{9}}+\frac{1}{z^{9} x^{2} y}\right)+ \\
& +5600\left(\frac{1}{z^{8} x^{2} y^{2}}+\frac{1}{z^{2} x^{2} y^{8}}+\frac{1}{z^{2} x^{8} y^{2}}\right)+ \\
& +6720\left(\frac{1}{z^{8} x^{3} y}+\frac{1}{x^{3} z y^{8}}+\frac{1}{x^{8} z y^{3}}+\frac{1}{z^{3} x y^{8}}+\frac{1}{z^{8} x y^{3}}+\frac{1}{z^{3} x^{8} y}\right)+ \\
& +6600\left(\frac{1}{z^{5} x^{6} y}+\frac{1}{z^{6} x y^{5}}+\frac{1}{z^{6} x^{5} y}+\frac{1}{z^{5} x y^{6}}+\frac{1}{x^{6} z y^{5}}+\frac{1}{x^{5} z y^{6}}\right)+ \\
& +6480\left(\frac{1}{z^{4} x^{3} y^{5}}+\frac{1}{z^{4} x^{5} y^{3}}+\frac{1}{z^{3} x^{5} y^{4}}+\frac{1}{z^{3} x^{4} y^{5}}+\right.
\end{aligned}
$$




$$
\left.\left.+\frac{1}{z^{5} x^{4} y^{3}}+\frac{1}{z^{5} x^{3} y^{4}}\right)+\frac{6336}{z^{4} x^{4} y^{4}}\right] N^{3}+O\left(N^{4}\right) .
$$

Четырехточечные функции:

$$
\begin{aligned}
& \rho_{G}^{(0 \mid 4)}(x, y, z, w)=\left[8\left(\frac{1}{w^{2} x y^{2} z}+\frac{1}{x^{2} y w z^{2}}+\frac{1}{w^{2} x^{2} y z}+\frac{1}{x y^{2} w z^{2}}+\frac{1}{w^{2} x y z^{2}}+\frac{1}{x^{2} y^{2} w z}\right)+\right. \\
& \left.+6\left(\frac{1}{x y^{3} w z}+\frac{1}{x y w z^{3}}+\frac{1}{w^{3} x y z}+\frac{1}{y x^{3} w z}\right)\right] N+ \\
& +\left[7 2 \left(\frac{1}{w^{3} x^{2} y z^{2}}+\frac{1}{w^{2} x y^{3} z^{2}}+\frac{1}{w^{2} x y^{2} z^{3}}+\frac{1}{w^{3} x y^{2} z^{2}}+\frac{1}{w^{2} x^{3} y^{2} z}+\frac{1}{x^{3} y^{2} w z^{2}}+\right.\right. \\
& +\frac{1}{w^{2} x^{3} y z^{2}}+\frac{1}{w^{2} x^{2} y^{3} z}+\frac{1}{x^{2} y^{3} w z^{2}}+\frac{1}{w^{3} x^{2} y^{2} z}+\frac{1}{x^{2} y^{2} w z^{3}}+\frac{1}{w^{2} x^{2} y z^{3}}+ \\
& +\frac{1}{x^{2} y w z^{4}}+\frac{1}{x y^{4} w z^{2}}+\frac{1}{w^{2} x y^{4} z}+\frac{1}{w^{4} x y z^{2}}+\frac{1}{w^{2} x y z^{4}}+\frac{1}{w^{2} x^{4} y z}+ \\
& +\frac{1}{w^{4} x y^{2} z}+\frac{1}{x y^{2} w z^{4}}+\frac{1}{x^{4} y^{2} w z}+\frac{1}{x^{2} y^{4} w z}+\frac{1}{w^{4} x^{2} y z}+\frac{1}{x^{4} y w z^{2}}+ \\
& \left.+\frac{1}{w^{3} x y^{3} z}+\frac{1}{x y^{3} w z^{3}}+\frac{1}{w^{3} x y z^{3}}+\frac{1}{x^{3} y^{3} w z}+\frac{1}{w^{3} x^{3} y z}+\frac{1}{x^{3} y w z^{3}}\right)+ \\
& \left.+60\left(\frac{1}{y x^{5} w z}+\frac{1}{x y w z^{5}}+\frac{1}{w^{5} x y z}+\frac{1}{x y^{5} w z}\right)+\frac{48}{w^{2} x^{2} y^{2} z^{2}}\right] N^{2}+ \\
& +\left[5 7 6 \left(\frac{1}{w^{3} x^{3} y^{2} z^{2}}+\frac{1}{w^{2} x^{3} y^{3} z^{2}}+\frac{1}{w^{3} x^{2} y^{3} z^{2}}+\frac{1}{w^{2} x^{2} y^{3} z^{3}}+\frac{1}{w^{2} x^{3} y^{2} z^{3}}+\right.\right. \\
& +\frac{1}{w^{3} x^{2} y^{2} z^{3}}+\frac{1}{w^{3} x^{2} y z^{4}}+\frac{1}{w^{4} x^{3} y^{2} z}+\frac{1}{x^{3} y^{2} w z^{4}}+\frac{1}{x^{4} y^{2} w z^{3}}+\frac{1}{w^{3} x^{4} y^{2} z}+ \\
& +\frac{1}{w^{2} x^{4} y^{3} z}+\frac{1}{x^{4} y^{3} w z^{2}}+\frac{1}{w^{4} x y^{2} z^{3}}+\frac{1}{w^{4} x^{3} y z^{2}}+\frac{1}{w^{2} x y^{4} z^{3}}+\frac{1}{x^{2} y^{4} w z^{3}}+ \\
& +\frac{1}{w^{3} x y^{4} z^{2}}+\frac{1}{w^{3} x^{4} y z^{2}}+\frac{1}{w^{2} x^{4} y z^{3}}+\frac{1}{w^{2} x y^{3} z^{4}}+\frac{1}{w^{4} x y^{3} z^{2}}+\frac{1}{w^{3} x y^{2} z^{4}}+ \\
& +\frac{1}{w^{4} x^{2} y^{3} z}+\frac{1}{w^{4} x^{2} y z^{3}}+\frac{1}{w^{2} x^{3} y z^{4}}+\frac{1}{w^{2} x^{3} y^{4} z}+\frac{1}{x^{3} y^{4} w z^{2}}+\frac{1}{w^{3} x^{2} y^{4} z}+ \\
& \left.+\frac{1}{x^{2} y^{3} w z^{4}}\right)+480\left(\frac{1}{w^{2} x^{6} y z}+\frac{1}{x y^{6} w z^{2}}+\frac{1}{w^{6} x y^{2} z}+\frac{1}{w^{6} x y z^{2}}+\right. \\
& +\frac{1}{x^{6} y w z^{2}}+\frac{1}{x^{6} y^{2} w z}+\frac{1}{w^{2} x y z^{6}}+\frac{1}{x y^{2} w z^{6}}+\frac{1}{w^{6} x^{2} y z}+\frac{1}{x^{2} y w z^{6}}+ \\
& +\frac{1}{w^{2} x y^{6} z}+\frac{1}{x^{2} y^{6} w z}+\frac{1}{w^{5} x y^{2} z^{2}}+\frac{1}{w^{5} x^{2} y z^{2}}+\frac{1}{x^{2} y^{2} w z^{5}}+\frac{1}{x^{2} y^{5} w z^{2}}+ \\
& +\frac{1}{w^{2} x^{2} y z^{5}}+\frac{1}{w^{2} x^{5} y z^{2}}+\frac{1}{w^{5} x^{2} y^{2} z}+\frac{1}{w^{2} x^{2} y^{5} z}+\frac{1}{w^{2} x^{5} y^{2} z}+ \\
& \left.+\frac{1}{x^{5} y^{2} w z^{2}}+\frac{1}{w^{2} x y^{5} z^{2}}+\frac{1}{w^{2} x y^{2} z^{5}}\right)+540\left(\frac{1}{x^{3} y w z^{5}}+\frac{1}{x y^{5} w z^{3}}+\frac{1}{w^{5} x^{3} y z}+\right. \\
& +\frac{1}{w^{5} x y z^{3}}+\frac{1}{w^{3} x y^{5} z}+\frac{1}{x^{5} y w z^{3}}+\frac{1}{x^{5} y^{3} w z}+\frac{1}{w^{3} x^{5} y z}+
\end{aligned}
$$




$$
\begin{aligned}
& \left.+\frac{1}{w^{5} x y^{3} z}+\frac{1}{x^{3} y^{5} w z}+\frac{1}{x y^{3} w z^{5}}+\frac{1}{w^{3} x y z^{5}}\right)+ \\
& +420\left(\frac{1}{x y^{7} w z}+\frac{1}{y x^{7} w z}+\frac{1}{x y w z^{7}}+\frac{1}{w^{7} x y z}\right)+ \\
& +648\left(\frac{1}{w^{3} x^{3} y^{3} z}+\frac{1}{w^{3} x y^{3} z^{3}}+\frac{1}{x^{3} y^{3} w z^{3}}+\frac{1}{w^{3} x^{3} y z^{3}}\right)+ \\
& +384\left(\frac{1}{w^{2} x^{2} y^{2} z^{4}}+\frac{1}{w^{4} x^{2} y^{2} z^{2}}+\frac{1}{w^{2} x^{4} y^{2} z^{2}}+\frac{1}{w^{2} x^{2} y^{4} z^{2}}\right)+ \\
& +576\left(\frac{1}{x^{4} y^{4} w z}+\frac{1}{w^{4} x^{4} y z}+\frac{1}{x^{4} y w z^{4}}+\frac{1}{w^{4} x y^{4} z}+\right. \\
& \left.\left.+\frac{1}{w^{4} x y z^{4}}+\frac{1}{x y^{4} w z^{4}}\right)\right] N^{3}+O\left(N^{4}\right) .
\end{aligned}
$$

1.6. Рекуррентные формулы для гауссовых корреляционных функций. Обшая рекуррентная формула для связных корреляционных функций, содержаших $\operatorname{tr} \phi$, имеет вид ${ }^{12)}$

$$
\begin{aligned}
\left\langle(\operatorname{tr} \phi)^{k} \operatorname{tr} \phi^{i_{1}} \ldots \operatorname{tr} \phi^{i_{m}}\right\rangle_{G}= & k ! \sum_{\substack{0 \leqslant a_{1}, \ldots, a_{m} \leqslant k \\
a_{1}+\cdots+a_{m}=k}} \frac{i_{1} !}{a_{1} !\left(i_{1}-a_{1}\right) !} \cdots \frac{i_{m} !}{a_{m} !\left(i_{m}-a_{m}\right) !} \times \\
& \times\left\langle\left\langle\operatorname{tr} \phi^{i_{1}-a_{1}} \ldots \operatorname{tr} \phi^{i_{m}-a_{m}}\right\rangle_{G} .\right.
\end{aligned}
$$

Некоторые примеры:

$$
\begin{aligned}
& \left\langle\left\langle(\operatorname{tr} \phi)^{k}\right\rangle\right\rangle_{G}=\delta_{k, 0}+N \delta_{k, 2}, \\
& \left\langle\left\langle(\operatorname{tr} \phi)^{k} \operatorname{tr} \phi^{2}\right\rangle\right\rangle_{G}=N^{2} \delta_{k, 0}+2 N \delta_{k, 2}, \\
& \left\langle\left\langle(\operatorname{tr} \phi)^{k} \operatorname{tr} \phi^{3}\right\rangle\right\rangle_{G}=3 N^{2} \delta_{k, 1}+6 N \delta_{k, 3}, \\
& \left\langle\left\langle(\operatorname{tr} \phi)^{k} \operatorname{tr} \phi^{4}\right\rangle\right\rangle_{G}=\left(2 N^{3}+N\right) \delta_{k, 0}+12 N^{2} \delta_{k, 2}+24 N \delta_{k, 4}, \\
& \left\langle\left\langle(\operatorname{tr} \phi)^{k} \operatorname{tr} \phi^{k}\right\rangle\right\rangle_{G}=N k ! \\
& \left\langle\left\langle(\operatorname{tr} \phi)^{k} \operatorname{tr} \phi^{m}\right\rangle\right\rangle_{G}=0 \quad \text { для } k>m .
\end{aligned}
$$

Вообше говоря, для $k+1$-точечных корреляционных функций

$$
\begin{aligned}
\left\langle\left\langle(\operatorname{tr} \phi)^{k} \operatorname{tr} \phi^{2 m}\right\rangle_{G}=\right. & c_{2 m} \delta_{k, 0}+2 m(2 m-1) c_{2 m-2} \delta_{k, 2}+\cdots+(2 m) ! N \delta_{k, 2 m}, \\
\left\langle\left\langle(\operatorname{tr} \phi)^{k} \operatorname{tr} \phi^{2 m+1}\right\rangle_{G}=\right. & (2 m+1) c_{2 m} \delta_{k, 1}+ \\
& +2 m\left(4 m^{2}-1\right) c_{2 m-2} \delta_{k, 3}+\cdots+(2 m+1) ! N \delta_{k, 2 m+1}, \\
\text { где } c_{2 m}=\left\langle\left\langle\operatorname{tr} \phi^{2 k}\right\rangle_{G} .\right. &
\end{aligned}
$$

${ }^{12)}$ При использовании этой формулы нужно проявлять осторожность: если некоторые $i_{\alpha}-$ $a_{\alpha}=0$, хотя некоторые другие $i_{\beta}-a_{\beta}>0$, то соответствующие связные корреляционные функции в правой части равенства обращаются в нуль: $\left\langle\left\langle\operatorname{tr} I \ldots \operatorname{tr} \phi^{i \beta}-a_{\beta} \ldots\right\rangle_{G}=0\right.$. 
Несвязные корреляционные функции имеют вид

$$
\left\langle(\operatorname{tr} \phi)^{2 k}\right\rangle_{G}=(2 k-1) ! ! N^{k} .
$$

Гауссовы корреляционные функции от величин $\operatorname{tr} \phi^{2}$ :

$$
\begin{aligned}
\left\langle\left\langle\left(\operatorname{tr} \phi^{2}\right)^{k}\right\rangle_{G}\right. & =4(k-1)(k-2)\left\langle\left\langle\left(\operatorname{tr} \phi^{2}\right)^{k-2}\right\rangle_{G}=2^{k-1}(k-1) ! N^{2},\right. \\
\left\langle\left(\operatorname{tr} \phi^{2}\right)^{k}\right\rangle_{G} & =N^{2}\left\langle\left(\operatorname{tr} \phi^{2}\right)^{k-1}\right\rangle_{G}+2(k-1)\left[N^{2}+2(k-2)\right]\left\langle\left(\operatorname{tr} \phi^{2}\right)^{k-2}\right\rangle_{G}= \\
& =\sum_{j=1}^{k} \frac{1}{j !} \sum_{\substack{0<l_{1}, \ldots, l_{j}<k \\
l_{1}+\cdots+l_{j}=k}} \frac{k !}{l_{1} ! \ldots l_{j} !}\left\langle\left(\operatorname{tr} \phi^{2}\right)^{l_{1}}\right\rangle_{G} \ldots\left\langle\left\langle\left(\operatorname{tr} \phi^{2}\right)^{l_{j}}\right\rangle_{G}=\right. \\
& =\sum_{j=1}^{k} \frac{2^{k-j} k !}{j !} N^{2 j} \sum_{\substack{0<l_{1}, \ldots, l_{j}<k \\
l_{1}+\cdots+l_{j}=k}} \frac{1}{l_{1} \cdot \ldots \cdot l_{j}} .
\end{aligned}
$$

Некоторые примеры:

$$
\begin{aligned}
\left\langle\operatorname{tr} \phi^{2}\right\rangle_{G} & =N^{2}, \\
\left\langle\left(\operatorname{tr} \phi^{2}\right)^{2}\right\rangle_{G} & =N^{4}+2 N^{2}, \\
\left\langle\left(\operatorname{tr} \phi^{2}\right)^{3}\right\rangle_{G} & =N^{6}+6 N^{4}+8 N^{2}, \\
\left\langle\left(\operatorname{tr} \phi^{2}\right)^{4}\right\rangle_{G} & =N^{8}+12 N^{6}+44 N^{4}+48 N^{2}, \\
\left\langle\left(\operatorname{tr} \phi^{2}\right)^{5}\right\rangle_{G} & =N^{10}+20 N^{8}+140 N^{6}+400 N^{4}+384 N^{2} .
\end{aligned}
$$

Рекуррентные формулы для корреляционных функций с $\operatorname{tr} \phi^{k}$ при $k \geqslant 2$ сложнее с точки зрения зависимости от $N$ (разложения по родам). Конкретные примеры с $\operatorname{tr} \phi^{2}$ см. в формулах (III.1.2) и (III.1.3).

1.7. Младшие гауссовы корреляционные функции, связные и несвязные. Для того чтобы восстановить зависимость от $g$ и $M$ в приведенных ниже формулах, каждую корреляционную функцию $\left\langle\operatorname{tr} \phi^{k_{1}} \ldots \operatorname{tr} \phi^{k_{m}}\right\rangle$ нужно умножить на $(g / M)^{\frac{r_{1}+\cdots+k_{m}}{2}}$.

Правила отбора:

а) для несвязны $x$ корреляционных функций при $N=1$

$$
\left\langle\operatorname{tr} \phi^{i_{1}} \ldots \operatorname{tr} \phi^{i_{k}}\right\rangle_{G}=\left(i_{1}+\cdots+i_{k}-1\right) ! !
$$

б) чтобы коррелятор не обращался в нуль, сумма $i_{1}+\cdots+i_{k}$ должна быть четной.

Одноточечные корреляционные функции (обобщенные числа Каталана):

$$
\begin{aligned}
\left\langle\operatorname{tr} \phi^{2}\right\rangle_{G} & =\left\langle\left\langle\operatorname{tr} \phi^{2}\right\rangle\right\rangle_{G}=N^{2}, \\
\left\langle\operatorname{tr} \phi^{4}\right\rangle_{G} & =\left\langle\left\langle\operatorname{tr} \phi^{4}\right\rangle\right\rangle_{G}=2 N^{3}+N, \\
\left\langle\operatorname{tr} \phi^{6}\right\rangle_{G} & =\left\langle\left\langle\operatorname{tr} \phi^{6}\right\rangle\right\rangle_{G}=5 N^{4}+10 N^{2}, \\
\left\langle\operatorname{tr} \phi^{8}\right\rangle_{G} & =\left\langle\left\langle\operatorname{tr} \phi^{8}\right\rangle\right\rangle_{G}=14 N^{5}+70 N^{3}+21 N, \\
\left\langle\operatorname{tr} \phi^{10}\right\rangle_{G} & =\left\langle\left\langle\operatorname{tr} \phi^{10}\right\rangle_{G}=42 N^{6}+420 N^{4}+483 N^{2} .\right.
\end{aligned}
$$


Двухточечные коррелящионные функции:

$$
\begin{aligned}
\left\langle\left\langle(\operatorname{tr} \phi)^{2}\right\rangle\right\rangle_{G} & =\left\langle(\operatorname{tr} \phi)^{2}\right\rangle_{G}=N \\
\left\langle\left\langle\left(\operatorname{tr} \phi^{2}\right)^{2}\right\rangle\right\rangle_{G} & =2 N^{2} \\
\left\langle\left(\operatorname{tr} \phi^{2}\right)^{2}\right\rangle_{G} & =N^{4}+2 N^{2} \\
\left\langle\left\langle\left(\operatorname{tr} \phi^{3}\right)^{2}\right\rangle\right\rangle_{G} & =\left\langle\left(\operatorname{tr} \phi^{3}\right)^{2}\right\rangle_{G}=12 N^{3}+3 N \\
\left\langle\left\langle\left(\operatorname{tr} \phi^{4}\right)^{2}\right\rangle\right\rangle_{G} & =36 N^{4}+60 N^{2} \\
\left\langle\left(\operatorname{tr} \phi^{4}\right)^{2}\right\rangle_{G} & =4 N^{6}+40 N^{4}+61 N^{2} \\
\left\langle\left\langle\left(\operatorname{tr} \phi^{5}\right)^{2}\right\rangle\right\rangle_{G} & =\left\langle\left(\operatorname{tr} \phi^{5}\right)^{2}\right\rangle_{G}=180 N^{5}+600 N^{3}+165 N, \\
\left\langle\left\langle\operatorname{tr} \phi \operatorname{tr} \phi^{3}\right\rangle\right\rangle_{G} & =\left\langle\operatorname{tr} \phi \operatorname{tr} \phi^{3}\right\rangle_{G}=3 N^{2} \\
\left\langle\left\langle\operatorname{tr} \phi \operatorname{tr} \phi^{5}\right\rangle\right\rangle_{G} & =\left\langle\operatorname{tr} \phi \operatorname{tr} \phi^{5}\right\rangle_{G}=10 N^{3}+5 N \\
\left\langle\left\langle\operatorname{tr} \phi \operatorname{tr} \phi^{7}\right\rangle\right\rangle_{G} & =\left\langle\operatorname{tr} \phi \operatorname{tr} \phi^{7}\right\rangle_{G}=35 N^{4}+70 N^{2} \\
\left\langle\left\langle\operatorname{tr} \phi \operatorname{tr} \phi^{9}\right\rangle\right\rangle_{G} & =\left\langle\operatorname{tr} \phi \operatorname{tr} \phi^{9}\right\rangle_{G}=126 N^{5}+630 N^{3}+189 N, \\
\left\langle\left\langle\operatorname{tr} \phi^{2} \operatorname{tr} \phi^{4}\right\rangle\right\rangle_{G} & =8 N^{3}+4 N \\
\left\langle\operatorname{tr} \phi^{2} \operatorname{tr} \phi^{4}\right\rangle_{G} & =2 N^{5}+9 N^{3}+4 N \\
\left\langle\left\langle\operatorname{tr} \phi^{2} \operatorname{tr} \phi^{6}\right\rangle\right\rangle_{G} & =30 N^{4}+60 N^{2} \\
\left\langle\operatorname{tr} \phi^{2} \operatorname{tr} \phi^{6}\right\rangle_{G} & =5 N^{6}+40 N^{4}+60 N^{2} \\
\left\langle\left\langle\operatorname{tr} \phi^{2} \operatorname{tr} \phi^{8}\right\rangle\right\rangle_{G} & =112 N^{5}+560 N^{3}+178 N \\
\left\langle\operatorname{tr} \phi^{2} \operatorname{tr} \phi^{8}\right\rangle_{G} & =14 N^{7}+182 N^{5}+581 N^{3}+178 N \\
\left\langle\left\langle\operatorname{tr} \phi^{3} \operatorname{tr} \phi^{5}\right\rangle\right\rangle_{G} & =\left\langle\operatorname{tr} \phi^{3} \operatorname{tr} \phi^{5}\right\rangle_{G}=45 N^{4}+60 N^{2} \\
\left\langle\left\langle\operatorname{tr} \phi^{3} \operatorname{tr} \phi^{7}\right\rangle\right\rangle_{G} & =\left\langle\operatorname{tr} \phi^{3} \operatorname{tr} \phi^{7}\right\rangle_{G}=168 N^{5}+630 N^{3}+147 N \\
\left\langle\left\langle\operatorname{tr} \phi^{4} \operatorname{tr} \phi^{6}\right\rangle\right\rangle_{G} & =144 N^{5}+600 N^{3}+156 N \\
\left\langle\operatorname{tr} \phi^{4} \operatorname{tr} \phi^{6}\right\rangle_{G} & =10 N^{7}+169 N^{5}+610 N^{3}+156 N .
\end{aligned}
$$

Трехточечные корреляционные функции:

$$
\begin{aligned}
\left\langle\left\langle\left(\operatorname{tr} \phi^{2}\right)^{3}\right\rangle\right\rangle_{G} & =8 N^{2}, \\
\left\langle\left(\operatorname{tr} \phi^{2}\right)^{3}\right\rangle_{G} & =N^{6}+6 N^{4}+8 N^{2}, \\
\left\langle\left\langle(\operatorname{tr} \phi)^{2} \operatorname{tr} \phi^{2}\right\rangle\right\rangle_{G} & =2 N, \\
\left\langle(\operatorname{tr} \phi)^{2} \operatorname{tr} \phi^{2}\right\rangle_{G} & =N^{3}+2 N, \\
\left\langle\left\langle(\operatorname{tr} \phi)^{2} \operatorname{tr} \phi^{4}\right\rangle\right\rangle_{G} & =12 N^{2}, \\
\left\langle(\operatorname{tr} \phi)^{2} \operatorname{tr} \phi^{4}\right\rangle_{G} & =2 N^{4}+13 N^{2}, \\
\left\langle\left\langle(\operatorname{tr} \phi)^{2} \operatorname{tr} \phi^{6}\right\rangle\right\rangle_{G} & =60 N^{3}+30 N, \\
\left\langle(\operatorname{tr} \phi)^{2} \operatorname{tr} \phi^{6}\right\rangle_{G} & =5 N^{5}+70 N^{3}+30 N, \\
\left\langle\left\langle(\operatorname{tr} \phi)^{2} \operatorname{tr} \phi^{8}\right\rangle\right\rangle_{G} & =280 N^{4}+560 N^{2}, \\
\left\langle(\operatorname{tr} \phi)^{2} \operatorname{tr} \phi^{8}\right\rangle_{G} & =14 N^{6}+350 N^{4}+581 N^{2}, \\
\left\langle\left\langle\left(\operatorname{tr} \phi^{2}\right)^{2} \operatorname{tr} \phi^{4}\right\rangle\right\rangle_{G} & =48 N^{3}+24 N,
\end{aligned}
$$




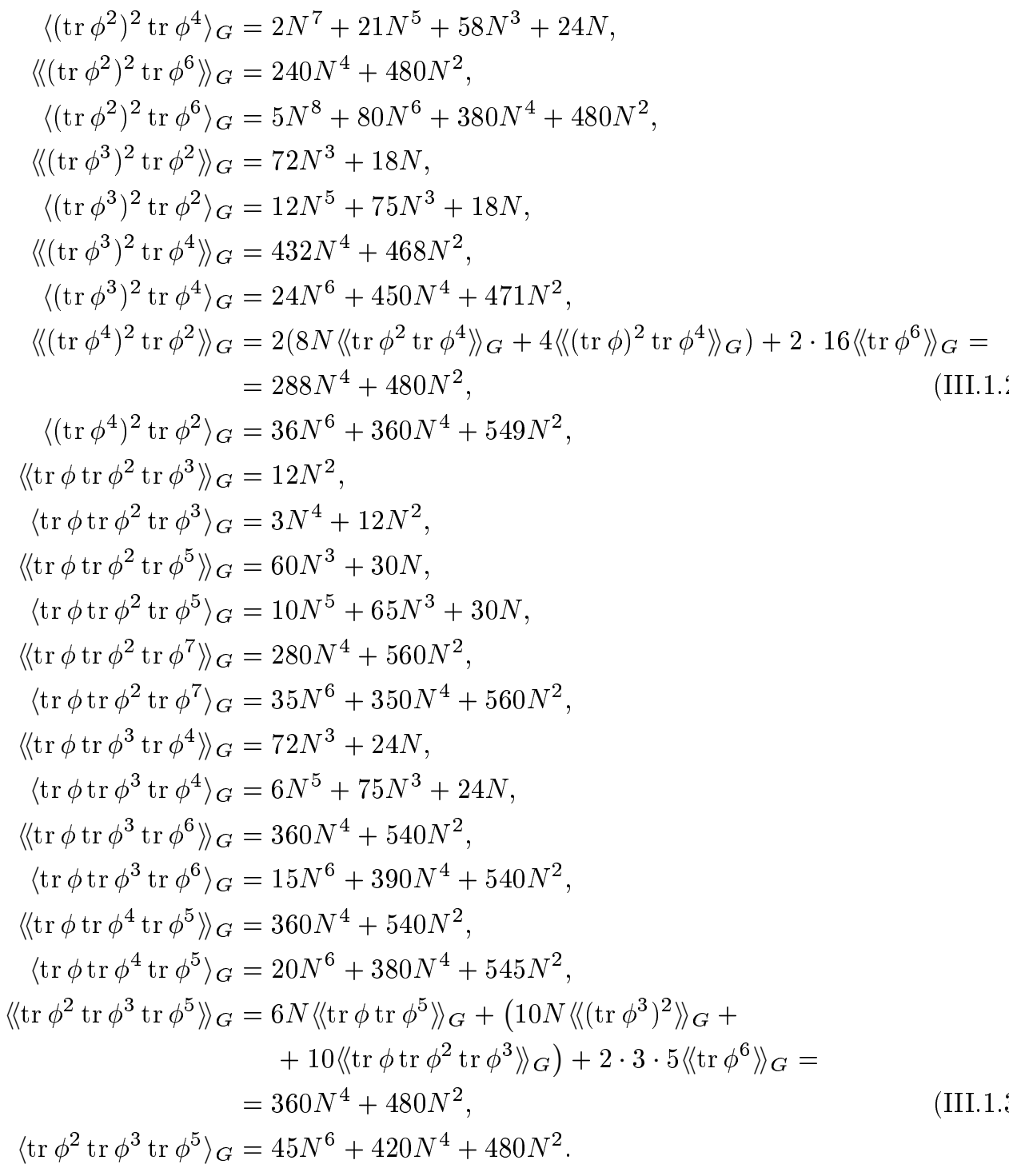

Четырехточечные корреляционные функции:

$$
\begin{aligned}
\left\langle\left\langle(\operatorname{tr} \phi)^{4}\right\rangle\right\rangle_{G} & =0, \\
\left\langle(\operatorname{tr} \phi)^{4}\right\rangle_{G} & =3 N^{2}, \\
\left\langle\left\langle\left(\operatorname{tr} \phi^{2}\right)^{4}\right\rangle\right\rangle_{G} & =48 N^{2}, \\
\left\langle\left(\operatorname{tr} \phi^{2}\right)^{4}\right\rangle_{G} & =N^{8}+12 N^{6}+44 N^{4}+48 N^{2}, \\
\left\langle\left\langle(\operatorname{tr} \phi)^{3} \operatorname{tr} \phi^{3}\right\rangle_{G}\right. & =6 N, \\
\left\langle(\operatorname{tr} \phi)^{3} \operatorname{tr} \phi^{3}\right\rangle_{G} & =9 N^{3}+6 N, \\
\left\langle\left\langle(\operatorname{tr} \phi)^{3} \operatorname{tr} \phi^{5}\right\rangle\right\rangle_{G} & =60 N^{2}
\end{aligned}
$$




$$
\begin{aligned}
& \left\langle(\operatorname{tr} \phi)^{3} \operatorname{tr} \phi^{5}\right\rangle_{G}=30 N^{4}+75 N^{2}, \\
& \left\langle\left\langle(\operatorname{tr} \phi)^{3} \operatorname{tr} \phi^{7}\right\rangle\right\rangle_{G}=420 N^{3}+210 N, \\
& \left\langle(\operatorname{tr} \phi)^{3} \operatorname{tr} \phi^{7}\right\rangle_{G}=105 N^{5}+630 N^{3}+210 N, \\
& \left\langle\left\langle\left(\operatorname{tr} \phi^{2}\right)^{3} \operatorname{tr} \phi^{4}\right\rangle_{G}=384 N^{3}+192 N,\right. \\
& \left\langle\left(\operatorname{tr} \phi^{2}\right)^{3} \operatorname{tr} \phi^{4}\right\rangle_{G}=2 N^{9}+37 N^{7}+226 N^{5}+488 N^{3}+192 N, \\
& \left\langle\left\langle\left(\operatorname{tr} \phi^{3}\right)^{3} \operatorname{tr} \phi\right\rangle\right\rangle_{G}=648 N^{3}+162 N, \\
& \left\langle\left(\operatorname{tr} \phi^{3}\right)^{3} \operatorname{tr} \phi\right\rangle_{G}=108 N^{5}+675 N^{3}+162 N, \\
& \left\langle\left\langle(\operatorname{tr} \phi)^{2}\left(\operatorname{tr} \phi^{2}\right)^{2}\right\rangle\right\rangle_{G}=8 N \text {, } \\
& \left\langle(\operatorname{tr} \phi)^{2}\left(\operatorname{tr} \phi^{2}\right)^{2}\right\rangle_{G}=N^{5}+6 N^{3}+8 N, \\
& \left\langle\left\langle(\operatorname{tr} \phi)^{2}\left(\operatorname{tr} \phi^{3}\right)^{2}\right\rangle\right\rangle_{G}=72 N^{2}, \\
& \left\langle(\operatorname{tr} \phi)^{2}\left(\operatorname{tr} \phi^{3}\right)^{2}\right\rangle_{G}=30 N^{4}+75 N^{2}, \\
& \left\langle\left\langle(\operatorname{tr} \phi)^{2}\left(\operatorname{tr} \phi^{4}\right)^{2}\right\rangle\right\rangle_{G}=576 N^{3}+192 N, \\
& \left\langle(\operatorname{tr} \phi)^{2}\left(\operatorname{tr} \phi^{4}\right)^{2}\right\rangle_{G}=4 N^{7}+88 N^{5}+661 N^{3}+192 N, \\
& \left\langle\left\langle\left(\operatorname{tr} \phi^{2}\right)^{2}\left(\operatorname{tr} \phi^{3}\right)^{2}\right\rangle_{G}=576 N^{3}+144 N,\right. \\
& \left\langle\left(\operatorname{tr} \phi^{2}\right)^{2}\left(\operatorname{tr} \phi^{3}\right)^{2}\right\rangle_{G}=12 N^{7}+171 N^{5}+618 N^{3}+144 N, \\
& \left\langle\left\langle(\operatorname{tr} \phi)^{2} \operatorname{tr} \phi^{2} \operatorname{tr} \phi^{4}\right\rangle\right\rangle_{G}=72 N^{2}, \\
& \left\langle(\operatorname{tr} \phi)^{2} \operatorname{tr} \phi^{2} \operatorname{tr} \phi^{4}\right\rangle_{G}=2 N^{6}+25 N^{4}+78 N^{2}, \\
& \left\langle\left\langle(\operatorname{tr} \phi)^{2} \operatorname{tr} \phi^{2} \operatorname{tr} \phi^{6}\right\rangle\right\rangle_{G}=480 N^{3}+240 N, \\
& \left\langle(\operatorname{tr} \phi)^{2} \operatorname{tr} \phi^{2} \operatorname{tr} \phi^{6}\right\rangle_{G}=5 N^{7}+110 N^{5}+590 N^{3}+240 N, \\
& \left\langle\left\langle\operatorname{tr} \phi\left(\operatorname{tr} \phi^{2}\right)^{2} \operatorname{tr} \phi^{3}\right\rangle_{G}=72 N^{2},\right. \\
& \left\langle\operatorname{tr} \phi\left(\operatorname{tr} \phi^{2}\right)^{2} \operatorname{tr} \phi^{3}\right\rangle_{G}=3 N^{6}+30 N^{4}+72 N^{2}, \\
& \left\langle\left\langle\operatorname{tr} \phi\left(\operatorname{tr} \phi^{2}\right)^{2} \operatorname{tr} \phi^{5}\right\rangle\right\rangle_{G}=480 N^{3}+240 N, \\
& \left\langle\operatorname{tr} \phi\left(\operatorname{tr} \phi^{2}\right)^{2} \operatorname{tr} \phi^{5}\right\rangle_{G}=10 N^{7}+145 N^{5}+550 N^{3}+240 N, \\
& \left\langle\left\langle(\operatorname{tr} \phi)^{2} \operatorname{tr} \phi^{3} \operatorname{tr} \phi^{5}\right\rangle\right\rangle_{G}=540 N^{3}+210 N \text {, } \\
& \left\langle(\operatorname{tr} \phi)^{2} \operatorname{tr} \phi^{3} \operatorname{tr} \phi^{5}\right\rangle_{G}=105 N^{5}+630 N^{3}+210 N, \\
& \left\langle\left\langle\operatorname{tr} \phi \operatorname{tr} \phi^{2} \operatorname{tr} \phi^{3} \operatorname{tr} \phi^{4}\right\rangle\right\rangle_{G}=576 N^{3}+192 N \text {, } \\
& \left\langle\operatorname{tr} \phi \operatorname{tr} \phi^{2} \operatorname{tr} \phi^{3} \operatorname{tr} \phi^{4}\right\rangle_{G}=126 N^{5}+627 N^{3}+192 N .
\end{aligned}
$$

Пятиточечные корреляционные функции:

$$
\begin{aligned}
\left\langle\left\langle\left(\operatorname{tr} \phi^{2}\right)^{5}\right\rangle_{G}\right. & =384 N^{2}, \\
\left\langle\left(\operatorname{tr} \phi^{2}\right)^{5}\right\rangle_{G} & =N^{10}+20 N^{8}+140 N^{6}+400 N^{4}+384 N^{2}, \\
\left\langle\left\langle(\operatorname{tr} \phi)^{4} \operatorname{tr} \phi^{2}\right\rangle\right\rangle_{G} & =0 \\
\left\langle(\operatorname{tr} \phi)^{4} \operatorname{tr} \phi^{2}\right\rangle_{G} & =3 N^{4}+12 N^{2}, \\
\left\langle\left\langle(\operatorname{tr} \phi)^{4} \operatorname{tr} \phi^{4}\right\rangle\right\rangle_{G} & =24 N \\
\left\langle(\operatorname{tr} \phi)^{4} \operatorname{tr} \phi^{4}\right\rangle_{G} & =6 N^{5}+75 N^{3}+24 N,
\end{aligned}
$$




$$
\begin{aligned}
\left\langle\left\langle(\operatorname{tr} \phi)^{3} \operatorname{tr} \phi^{2} \operatorname{tr} \phi^{3}\right\rangle\right\rangle_{G} & =36 N, \\
\left\langle(\operatorname{tr} \phi)^{3} \operatorname{tr} \phi^{2} \operatorname{tr} \phi^{3}\right\rangle_{G} & =9 N^{5}+60 N^{3}+36 N, \\
\left\langle\left\langle(\operatorname{tr} \phi)^{3} \operatorname{tr} \phi^{2} \operatorname{tr} \phi^{5}\right\rangle\right\rangle_{G} & =480 N^{2}, \\
\left\langle(\operatorname{tr} \phi)^{3} \operatorname{tr} \phi^{2} \operatorname{tr} \phi^{5}\right\rangle_{G} & =30 N^{6}+315 N^{4}+600 N^{2}, \\
\left\langle\left\langle(\operatorname{tr} \phi)^{3} \operatorname{tr} \phi^{3} \operatorname{tr} \phi^{4}\right\rangle\right\rangle_{G} & =504 N^{2}, \\
\left\langle(\operatorname{tr} \phi)^{3} \operatorname{tr} \phi^{3} \operatorname{tr} \phi^{4}\right\rangle_{G} & =18 N^{6}+345 N^{4}+582 N^{2}, \\
\left\langle\left\langle\left(\operatorname{tr} \phi^{2}\right)^{3}(\operatorname{tr} \phi)^{2}\right\rangle\right\rangle_{G} & =48 N, \\
\left\langle\left(\operatorname{tr} \phi^{2}\right)^{3}(\operatorname{tr} \phi)^{2}\right\rangle_{G} & =N^{7}+12 N^{5}+44 N^{3}+48 N, \\
\left.\left\langle(\operatorname{tr} \phi)^{2}\left(\operatorname{tr} \phi^{2}\right)^{2} \operatorname{tr} \phi^{4}\right\rangle\right\rangle_{G} & =384 N^{2}, \\
\left\langle(\operatorname{tr} \phi)^{2}\left(\operatorname{tr} \phi^{2}\right)^{2} \operatorname{tr} \phi^{4}\right\rangle_{G} & =2 N^{8}+41 N^{6}+278 N^{4}+624 N^{2} .
\end{aligned}
$$

Шеститочечные корреляционные функции:

$$
\begin{aligned}
\left\langle\left\langle(\operatorname{tr} \phi)^{6}\right\rangle_{G}\right. & =0, \\
\left\langle(\operatorname{tr} \phi)^{6}\right\rangle_{G} & =15 N^{3}, \\
\left\langle\left\langle(\operatorname{tr} \phi)^{5} \operatorname{tr} \phi^{3}\right\rangle_{G}\right. & =0, \\
\left\langle(\operatorname{tr} \phi)^{5} \operatorname{tr} \phi^{3}\right\rangle_{G} & =45 N^{4}+60 N, \\
\left\langle\left\langle(\operatorname{tr} \phi)^{5} \operatorname{tr} \phi^{5}\right\rangle_{G}\right. & =120 N, \\
\left\langle(\operatorname{tr} \phi)^{5} \operatorname{tr} \phi^{5}\right\rangle_{G} & =150 N^{5}+675 N^{3}+120 N, \\
\left\langle\left\langle(\operatorname{tr} \phi)^{4}\left(\operatorname{tr} \phi^{2}\right)^{2}\right\rangle_{G}\right. & =0, \\
\left\langle(\operatorname{tr} \phi)^{4}\left(\operatorname{tr} \phi^{2}\right)^{2}\right\rangle_{G} & =3 N^{6}+30 N^{4}+72 N^{2}, \\
\left\langle(\operatorname{tr} \phi)^{4}\left(\operatorname{tr} \phi^{3}\right)^{2}\right\rangle_{G} & =216 N \\
\left\langle(\operatorname{tr} \phi)^{4}\left(\operatorname{tr} \phi^{3}\right)^{2}\right\rangle_{G} & =144 N^{5}+585 N^{3}+216 N, \\
\left\langle\left\langle\left(\operatorname{tr} \phi^{2}\right)^{4}(\operatorname{tr} \phi)^{2}\right\rangle_{G}\right. & =384 N \\
\left\langle\left(\operatorname{tr} \phi^{2}\right)^{4}(\operatorname{tr} \phi)^{2}\right\rangle_{G} & =N^{9}+20 N^{7}+140 N^{5}+400 N^{3}+384 N, \\
\left\langle(\operatorname{tr} \phi)^{3}\left(\operatorname{tr} \phi^{2}\right)^{2} \operatorname{tr} \phi^{3}\right\rangle_{G} & =288 N, \\
\left\langle(\operatorname{tr} \phi)^{3}\left(\operatorname{tr} \phi^{2}\right)^{2} \operatorname{tr} \phi^{3}\right\rangle_{G} & =9 N^{7}+132 N^{5}+516 N^{3}+288 N .
\end{aligned}
$$

Семиточечные коррелящионные функции:

$$
\begin{aligned}
\left\langle\left\langle(\operatorname{tr} \phi)^{6} \operatorname{tr} \phi^{2}\right\rangle\right\rangle_{G} & =0, \\
\left\langle(\operatorname{tr} \phi)^{6} \operatorname{tr} \phi^{2}\right\rangle_{G} & =15 N^{5}+90 N^{3}, \\
\left\langle\left\langle(\operatorname{tr} \phi)^{6} \operatorname{tr} \phi^{4}\right\rangle\right\rangle_{G} & =0, \\
\left\langle(\operatorname{tr} \phi)^{6} \operatorname{tr} \phi^{4}\right\rangle_{G} & =30 N^{6}+555 N^{4}+360 N^{2}, \\
\left\langle\left\langle(\operatorname{tr} \phi)^{5} \operatorname{tr} \phi^{2} \operatorname{tr} \phi^{3}\right\rangle\right\rangle_{G} & =0, \\
\left\langle(\operatorname{tr} \phi)^{5} \operatorname{tr} \phi^{2} \operatorname{tr} \phi^{3}\right\rangle_{G} & =45 N^{6}+420 N^{4}+480 N^{2}, \\
\left\langle\left\langle(\operatorname{tr} \phi)^{4}\left(\operatorname{tr} \phi^{2}\right)^{3}\right\rangle\right\rangle_{G} & =0,
\end{aligned}
$$




$$
\left\langle(\operatorname{tr} \phi)^{4}\left(\operatorname{tr} \phi^{2}\right)^{3}\right\rangle_{G}=3 N^{8}+54 N^{6}+312 N^{4}+576 N^{2} .
$$

Восьмиточечные корреляционные функции:

$$
\begin{aligned}
\left\langle\left\langle(\operatorname{tr} \phi)^{8}\right\rangle_{G}\right. & =0 \\
\left\langle(\operatorname{tr} \phi)^{8}\right\rangle_{G} & =105 N \\
\left.\left\langle(\operatorname{tr} \phi)^{7} \operatorname{tr} \phi^{3}\right\rangle\right\rangle_{G} & =0 \\
\left\langle(\operatorname{tr} \phi)^{7} \operatorname{tr} \phi^{3}\right\rangle_{G} & =315 N^{5}+630 N^{3} \\
\left\langle\left\langle(\operatorname{tr} \phi)^{6}\left(\operatorname{tr} \phi^{2}\right)^{2}\right\rangle\right\rangle_{G} & =0 \\
\left\langle(\operatorname{tr} \phi)^{6}\left(\operatorname{tr} \phi^{2}\right)^{2}\right\rangle_{G} & =15 N^{7}+210 N^{5}+720 N^{3}
\end{aligned}
$$

Девятиточечные корреляционные функции:

$$
\begin{aligned}
\left\langle\left\langle(\operatorname{tr} \phi)^{8} \operatorname{tr} \phi^{2}\right\rangle\right\rangle_{G} & =0, \\
\left\langle(\operatorname{tr} \phi)^{8} \operatorname{tr} \phi^{2}\right\rangle_{G} & =105 N^{6}+840 N^{4} .
\end{aligned}
$$

Десятиточечные корреляционные функции:

$$
\begin{aligned}
\left\langle\left\langle(\operatorname{tr} \phi)^{10}\right\rangle_{G}\right. & =0, \\
\left\langle(\operatorname{tr} \phi)^{10}\right\rangle_{G} & =945 N .
\end{aligned}
$$

1.8. Младшие препотенциалы $\mathcal{F}^{(p)}$ : первые члены разложения по $t$. Препотенциал - это производящая функция для связных корреляционных функций

$$
\begin{aligned}
\mathcal{F}_{G}= & N t_{0}+\sum_{k=1}^{\infty} t_{k}\left\langle\left\langle\operatorname{tr} \phi^{k}\right\rangle_{G}+\frac{1}{2 !} \sum_{k_{1}, k_{2}=0}^{\infty} t_{k_{1}} t_{k_{2}}\left\langle\left\langle\operatorname{tr} \phi^{k_{1}} \operatorname{tr} \phi^{k_{2}}\right\rangle_{G}+\right.\right. \\
& +\frac{1}{6 !} \sum_{k_{1}, k_{2}, k_{3}=0}^{\infty} t_{k_{1}} t_{k_{2}} t_{k_{3}}\left\langle\left\langle\operatorname{tr} \phi^{k_{1}} \operatorname{tr} \phi^{k_{2}} \operatorname{tr} \phi^{k_{3}}\right\rangle_{G}+\cdots\right.
\end{aligned}
$$

Для корреляционных функций, взятых из п. III.1.7, необходимо восстановить зависимость от $g$ и $M$ и сделать подстановку $N=M S / g$, так что

$$
\begin{aligned}
\mathcal{F}_{G}(t \mid S)= & \sum_{p \geqslant 0} g^{2 p-2} \mathcal{F}_{G}^{(p)}(t \mid S)= \\
= & \sum_{m=1}^{\infty} \frac{1}{m !} \sum_{k_{1}, \ldots, k_{m}=0}^{\infty} \frac{t_{k_{1}} \ldots t_{k_{m}}}{g^{m}}\left(\frac{g}{M}\right)^{\frac{k_{1}+\cdots+k_{m}}{2}}\left\langle\left\langle\operatorname{tr} \phi^{k_{1}} \ldots \operatorname{tr} \phi^{k_{m}}\right\rangle_{G}\left\langle\operatorname{tr} \phi^{2}\right\rangle_{G}=\right. \\
= & \frac{N t_{0}}{g}+\frac{N^{2} t_{2}}{M}+\frac{g\left(2 N^{3}+N\right) t_{4}}{M^{2}}+\frac{g^{2}\left(5 N^{4}+10 N^{2}\right) t_{6}}{M^{3}}+ \\
& +\frac{g^{3}\left(14 N^{5}+70 N^{3}+21 N\right) t_{8}}{M^{4}}+\frac{g^{4}\left(42 N^{6}+420 N^{4}+483 N^{2}\right) t_{10}}{M^{5}}+\cdots \\
& \cdots+\frac{N t_{1}^{2}}{2 g M}+\frac{N^{2} t_{2}^{2}}{M^{2}}+\frac{g\left(12 N^{3}+3 N\right) t_{3}^{2}}{2 M^{3}}+\frac{g^{2}\left(36 N^{4}+60 N^{2}\right) t_{4}^{2}}{2 M^{4}}+
\end{aligned}
$$




$$
\begin{aligned}
& +\frac{g^{3}\left(180 N^{5}+600 N^{3}+165 N\right) t_{5}^{2}}{2 M^{5}}+\cdots+\frac{3 N^{2} t_{1} t_{3}}{M^{2}}+\frac{g\left(10 N^{3}+5 N\right) t_{1} t_{5}}{M^{3}}+ \\
& +\frac{g^{2}\left(35 N^{4}+70 N^{2}\right) t_{1} t_{7}}{M^{4}}+\frac{g^{3}\left(126 N^{5}+630 N^{3}+189 N\right) t_{1} t_{9}}{M^{5}}+\cdots \\
& \cdots+\frac{g\left(8 N^{3}+4 N\right) t_{2} t_{4}}{M^{3}}+\frac{g^{2}\left(30 N^{4}+60 N^{2}\right) t_{2} t_{6}}{M^{4}}+ \\
& +\frac{g^{3}\left(112 N^{5}+560 N^{3}+178 N\right) t_{2} t_{8}}{M^{5}}+\cdots \\
& \cdots+\frac{g^{2}\left(45 N^{4}+60 N^{2}\right) t_{3} t_{5}}{M^{4}}+\frac{g^{3}\left(168 N^{5}+630 N^{3}+147 N\right) t_{3} t_{7}}{M^{5}}+\cdots \\
& \cdots+\frac{g^{3}\left(144 N^{5}+600 N^{3}+156 N\right) t_{4} t_{6}}{M^{5}}+\cdots+\frac{8 N^{2} t_{2}^{3}}{6 M^{3}}+\cdots+\frac{2 N t_{1}^{2} t_{2}}{2 g M^{2}}+ \\
& +\frac{12 N^{2} t_{1}^{2} t_{4}}{2 M^{3}}+\frac{g\left(60 N^{3}+30 N\right) t_{1}^{2} t_{6}}{2 M^{4}}+\frac{g^{2}\left(280 N^{4}+560 N^{2}\right) t_{1}^{2} t_{8}}{2 M^{5}}+\cdots \\
& \cdots+\frac{g\left(48 N^{3}+24 N\right) t_{2}^{2} t_{4}}{2 M^{4}}+\frac{g^{2}\left(240 N^{4}+480 N^{2}\right) t_{2}^{2} t_{6}}{2 M^{5}}+\cdots \\
& \cdots+\frac{g\left(72 N^{3}+18 N\right) t_{2} t_{3}^{2}}{2 M^{4}}+\frac{g^{2}\left(432 N^{4}+468 N^{2}\right) t_{3}^{2} t_{4}}{2 M^{5}}+\cdots \\
& \cdots+\frac{g^{2}\left(288 N^{4}+480 N^{2}\right) t_{2} t_{4}^{2}}{2 M^{5}}+\cdots \\
& \cdots+\frac{12 N^{2} t_{1} t_{2} t_{3}}{M^{3}}+\frac{g\left(60 N^{3}+30 N\right) t_{1} t_{2} t_{5}}{M^{4}}+\frac{g^{2}\left(280 N^{4}+560 N^{2}\right) t_{1} t_{2} t_{7}}{M^{5}}+\cdots \\
& \cdots+\frac{g\left(72 N^{3}+24 N\right) t_{1} t_{3} t_{4}}{M^{4}}+\frac{g^{2}\left(360 N^{4}+540 N^{2}\right) t_{1} t_{3} t_{6}}{M^{5}}+\cdots \\
& \cdots+\frac{g^{2}\left(360 N^{4}+540 N^{2}\right) t_{1} t_{4} t_{5}}{M^{5}}+\cdots \\
& \cdots+\frac{g^{2}\left(360 N^{4}+480 N^{2}\right) t_{2} t_{3} t_{5}}{M^{5}}+\cdots+\frac{48 N^{2} t_{2}^{4}}{24 M^{4}}+\cdots+\frac{6 N t_{1}^{3} t_{3}}{6 g M^{3}}+ \\
& +\frac{60 N^{2} t_{1}^{3} t_{5}}{6 M^{4}}+\frac{g\left(420 N^{3}+210 N\right) t_{1}^{3} t_{7}}{6 M^{5}}+\cdots+\frac{g\left(384 N^{3}+192 N\right) t_{2}^{3} t_{4}}{6 M^{5}}+\cdots \\
& \cdots+\frac{8 N t_{1}^{2} t_{2}^{2}}{4 g M^{3}}+\frac{72 N^{2} t_{1}^{2} t_{3}^{2}}{4 M^{4}}+\frac{g\left(576 N^{3}+192 N\right) t_{1}^{2} t_{4}^{2}}{4 M^{5}}+\cdots \\
& \cdots+\frac{g\left(576 N^{3}+144 N\right) t_{2}^{2} t_{3}^{2}}{4 M^{5}}+\cdots+\frac{72 N^{2} t_{1}^{2} t_{2} t_{4}}{2 M^{4}}+ \\
& +\frac{g\left(540 N^{3}+210 N\right) t_{1}^{2} t_{3} t_{5}}{2 M^{5}}+\frac{g\left(480 N^{3}+240 N\right) t_{1}^{2} t_{2} t_{6}}{2 M^{5}}+\cdots \\
& \cdots+\frac{72 N^{2} t_{1} t_{2}^{2} t_{3}}{2 M^{4}}+\frac{g\left(480 N^{3}+240 N\right) t_{1} t_{2}^{2} t_{5}}{2 M^{5}}+\cdots \\
& \cdots+\frac{g\left(576 N^{3}+192 N\right) t_{1} t_{2} t_{3} t_{4}}{M^{5}}+\cdots+\frac{384 N^{2} t_{2}^{5}}{120 M^{5}}+\cdots+\frac{24 N t_{1}^{4} t_{4}}{24 M^{5}}+\cdots \\
& \cdots+\frac{36 N t_{1}^{3} t_{2} t_{3}}{6 g M^{4}}+\frac{480 N^{2} t_{1}^{3} t_{2} t_{5}}{6 M^{5}}+\frac{504 N^{2} t_{1}^{3} t_{3} t_{4}}{6 M^{5}}+\cdots
\end{aligned}
$$




$$
\begin{aligned}
& \cdots+\frac{48 N t_{1}^{2} t_{2}^{3}}{12 g M^{4}}+\cdots+\frac{384 N^{2} t_{1}^{2} t_{2}^{2} t_{4}}{4 M^{5}}+\cdots+\frac{N t_{1}^{5} t_{5}}{g M^{5}}+\cdots \\
& \cdots+\frac{216 N t_{1}^{4} t_{3}^{2}}{48 g M^{5}}+\cdots+\frac{384 N t_{1}^{2} t_{2}^{4}}{48 g M^{5}}+\cdots+\frac{288 N t_{1}^{3} t_{2}^{2} t_{3}}{16 g M^{5}}+\cdots .
\end{aligned}
$$

1.9. Функции $Z_{G}(t \mid N)$ : первые члены разложения по $t-$

$$
\begin{aligned}
& e^{-\frac{N t_{0}}{g}} Z_{G}(t \mid N)=1+\frac{N^{2} t_{2}}{M}+\frac{g\left(2 N^{3}+N\right) t_{4}}{M^{2}}+\frac{g^{2}\left(5 N^{4}+10 N^{2}\right) t_{6}}{M^{3}}+ \\
& +\frac{g^{3}\left(14 N^{5}+70 N^{3}+21 N\right) t_{8}}{M^{4}}+\frac{g^{4}\left(42 N^{6}+420 N^{4}+483 N^{2}\right) t_{10}}{M^{5}}+\cdots \\
& \cdots+\frac{N t_{1}^{2}}{2 g M}+\frac{\left(N^{4}+2 N^{2}\right) t_{2}^{2}}{2 M^{2}}+\frac{g\left(12 N^{3}+3 N\right) t_{3}^{2}}{2 M^{3}}+ \\
& +\frac{g^{2}\left(4 N^{6}+40 N^{4}+61 N^{2}\right) t_{4}^{2}}{2 M^{4}}+\frac{g^{3}\left(180 N^{5}+600 N^{3}+165 N\right) t_{5}^{2}}{2 M^{5}}+\cdots \\
& \cdots+\frac{3 N^{2} t_{1} t_{3}}{M^{2}}+\frac{g\left(10 N^{3}+5 N\right) t_{1} t_{5}}{M^{3}}+\frac{g^{2}\left(35 N^{4}+70 N^{2}\right) t_{1} t_{7}}{M^{4}}+ \\
& +\frac{g^{3}\left(126 N^{5}+630 N^{3}+189 N\right) t_{1} t_{9}}{M^{5}}+\cdots \\
& \cdots+\frac{g\left(2 N^{5}+9 N^{3}+4 N\right) t_{2} t_{4}}{M^{3}}+\frac{g^{2}\left(5 N^{6}+40 N^{4}+60 N^{2}\right) t_{2} t_{6}}{M^{4}}+ \\
& +\frac{g^{3}\left(14 N^{7}+182 N^{5}+581 N^{3}+178 N\right) t_{2} t_{8}}{M^{5}}+\cdots+\frac{g^{2}\left(45 N^{4}+60 N^{2}\right) t_{3} t_{5}}{M^{4}}+ \\
& +\frac{g^{3}\left(168 N^{5}+630 N^{3}+147 N\right) t_{3} t_{7}}{M^{5}}+\cdots \\
& \cdots+\frac{g^{3}\left(10 N^{7}+169 N^{5}+610 N^{3}+156 N\right) t_{4} t_{6}}{M^{5}}+\cdots+\frac{\left(N^{6}+6 N^{4}+8 N^{2}\right) t_{2}^{3}}{6 M^{3}}+\cdots \\
& \cdots+\frac{\left(N^{3}+2 N\right) t_{1}^{2} t_{2}}{2 g M^{2}}+\frac{\left(2 N^{4}+13 N^{2}\right) t_{1}^{2} t_{4}}{2 M^{3}}+\frac{g\left(5 N^{5}+70 N^{3}+30 N\right) t_{1}^{2} t_{6}}{2 M^{4}}+ \\
& +\frac{g^{2}\left(14 N^{6}+350 N^{4}+581 N^{2}\right) t_{1}^{2} t_{8}}{2 M^{5}}+\cdots+\frac{g\left(2 N^{7}+21 N^{5}+58 N^{3}+24 N\right) t_{2}^{2} t_{4}}{2 M^{4}}+ \\
& +\frac{g^{2}\left(5 N^{8}+80 N^{6}+380 N^{4}+480 N^{2}\right) t_{2}^{2} t_{6}}{2 M^{5}}+\cdots \\
& \cdots+\frac{g\left(12 N^{5}+75 N^{3}+18 N\right) t_{2} t_{3}^{2}}{2 M^{4}}+\frac{g^{2}\left(24 N^{6}+450 N^{4}+471 N^{2}\right) t_{3}^{2} t_{4}}{2 M^{5}}+\cdots \\
& \cdots+\frac{g^{2}\left(36 N^{6}+360 N^{4}+549 N^{2}\right) t_{2} t_{4}^{2}}{2 M^{5}}+\cdots \\
& \cdots+\frac{\left(3 N^{4}+12 N^{2}\right) t_{1} t_{2} t_{3}}{M^{3}}+\frac{g\left(10 N^{5}+65 N^{3}+30 N\right) t_{1} t_{2} t_{5}}{M^{4}}+ \\
& +\frac{g^{2}\left(35 N^{6}+350 N^{4}+560 N^{2}\right) t_{1} t_{2} t_{7}}{M^{5}}+\cdots \\
& \cdots+\frac{g\left(6 N^{5}+75 N^{3}+24 N\right) t_{1} t_{3} t_{4}}{M^{4}}+\frac{g^{2}\left(15 N^{6}+390 N^{4}+540 N^{2}\right) t_{1} t_{3} t_{6}}{M^{5}}+\cdots \\
& \cdots+\frac{g^{2}\left(20 N^{6}+380 N^{4}+545 N^{2}\right) t_{1} t_{4} t_{5}}{M^{5}}+\cdots
\end{aligned}
$$




$$
\begin{aligned}
& \cdots+\frac{g^{2}\left(45 N^{6}+420 N^{4}+480 N^{2}\right) t_{2} t_{3} t_{5}}{M^{5}}+\cdots \\
& \cdots+\frac{3 N^{2} t_{1}^{4}}{24 g^{2} M^{2}}+\frac{\left(N^{8}+12 N^{6}+44 N^{4}+48 N^{2}\right) t_{2}^{4}}{24 M^{4}}+\cdots+\frac{\left(9 N^{3}+6 N\right) t_{1}^{3} t_{3}}{6 g M^{3}}+ \\
& +\frac{\left(30 N^{4}+75 N^{2}\right) t_{1}^{3} t_{5}}{6 M^{4}}+\frac{g\left(105 N^{5}+630 N^{3}+210 N\right) t_{1}^{3} t_{7}}{6 M^{5}}+\cdots \\
& \cdots+\frac{g\left(2 N^{9}+37 N^{7}+226 N^{5}+488 N^{3}+192 N\right) t_{2}^{3} t_{4}}{6 M^{5}}+\cdots \\
& \cdots+\frac{\left(N^{5}+6 N^{3}+8 N\right) t_{1}^{2} t_{2}^{2}}{4 g M^{3}}+\frac{\left(30 N^{4}+75 N^{2}\right) t_{1}^{2} t_{3}^{2}}{4 M^{4}}+ \\
& +\frac{g\left(4 N^{7}+88 N^{5}+661 N^{3}+192 N\right) t_{1}^{2} t_{4}^{2}}{4 M^{5}}+\cdots \\
& \cdots+\frac{g\left(12 N^{7}+171 N^{5}+618 N^{3}+144 N\right) t_{2}^{2} t_{3}^{2}}{4 M^{5}}+\cdots \\
& \cdots+\frac{\left(2 N^{6}+25 N^{4}+78 N^{2}\right) t_{1}^{2} t_{2} t_{4}}{2 M^{4}}+\frac{g\left(105 N^{5}+630 N^{3}+210 N\right) t_{1}^{2} t_{3} t_{5}}{2 M^{5}}+ \\
& +\frac{g\left(5 N^{7}+110 N^{5}+590 N^{3}+240 N\right) t_{1}^{2} t_{2} t_{6}}{2 M^{5}}+\cdots \\
& \cdots+\frac{\left(3 N^{6}+30 N^{4}+72 N^{2}\right) t_{1} t_{2}^{2} t_{3}}{2 M^{4}}+ \\
& +\frac{g\left(10 N^{7}+145 N^{5}+550 N^{3}+240 N\right) t_{1} t_{2}^{2} t_{5}}{2 M^{5}}+\cdots \\
& \cdots+\frac{g\left(126 N^{5}+627 N^{3}+192 N\right) t_{1} t_{2} t_{3} t_{4}}{M^{5}}+\cdots \\
& \cdots+\frac{\left(N^{10}+20 N^{8}+140 N^{6}+400 N^{4}+384 N^{2}\right) t_{2}^{5}}{120 M^{5}}+\cdots \\
& \cdots+\frac{\left(3 N^{4}+12 N^{2}\right) t_{1}^{4} t_{2}}{24 g M^{4}}+\frac{\left(6 N^{5}+75 N^{3}+24 N\right) t_{1}^{4} t_{4}}{24 M^{5}}+\cdots \\
& \cdots+\frac{\left(9 N^{5}+60 N^{3}+36 N\right) t_{1}^{3} t_{2} t_{3}}{6 g M^{4}}+\frac{\left(30 N^{6}+315 N^{4}+600 N^{2}\right) t_{1}^{3} t_{2} t_{5}}{6 M^{5}}+ \\
& +\frac{\left(18 N^{6}+345 N^{4}+582 N^{2}\right) t_{1}^{3} t_{3} t_{4}}{6 M^{5}}+\cdots+\frac{\left(N^{7}+12 N^{5}+44 N^{3}+48 N\right) t_{1}^{2} t_{2}^{3}}{12 g M^{4}}+\cdots \\
& \cdots+\frac{\left(2 N^{8}+41 N^{6}+278 N^{4}+624 N^{2}\right) t_{1}^{2} t_{2}^{2} t_{4}}{4 M^{5}}+\cdots+\frac{15 N^{3} t_{1}^{6}}{720 g^{3} M^{3}}+\cdots \\
& \cdots+\frac{\left(45 N^{4}+60 N\right) t_{1}^{5} t_{3}}{120 g^{2} M^{4}}+\frac{\left(150 N^{5}+675 N^{3}+120 N\right) t_{1}^{5} t_{5}}{120 g M^{5}}+\cdots \\
& \cdots+\frac{\left(3 N^{6}+30 N^{4}+72 N^{2}\right) t_{1}^{4} t_{2}^{2}}{48 g^{2} M^{4}}+\frac{\left(144 N^{5}+585 N^{3}+216 N\right) t_{1}^{4} t_{3}^{2}}{48 g M^{5}}+\cdots \\
& \cdots+\frac{\left(N^{9}+20 N^{7}+140 N^{5}+400 N^{3}+384 N\right) t_{1}^{2} t_{2}^{4}}{48 g M^{5}}+\cdots \\
& \cdots+\frac{\left(9 N^{7}+132 N^{5}+516 N^{3}+288 N\right) t_{1}^{3} t_{2}^{2} t_{3}}{16 g M^{5}}+\cdots
\end{aligned}
$$




$$
\begin{aligned}
& \cdots+\frac{\left(15 N^{5}+90 N^{3}\right) t_{1}^{6} t_{2}}{720 g^{3} M^{4}}+\frac{\left(30 N^{6}+555 N^{4}+360 N^{2}\right) t_{1}^{6} t_{4}}{720 g^{2} M^{5}}+\cdots \\
& \cdots+\frac{\left(45 N^{6}+420 N^{4}+480 N^{2}\right) t_{1}^{5} t_{2} t_{3}}{60 g^{2} M^{5}}+\cdots \\
& \cdots+\frac{\left(3 N^{8}+54 N^{6}+312 N^{4}+576 N^{2}\right) t_{1}^{4} t_{2}^{3}}{240 g^{2} M^{5}}+\cdots \\
& \cdots+\frac{105 N^{4} t_{1}^{8}}{5760 g^{4} M^{4}}+\cdots+\frac{\left(315 N^{5}+630 N^{3}\right) t_{1}^{7} t_{3}}{720 g^{3} M^{5}}+\cdots \\
& \cdots+\frac{\left(15 N^{7}+210 N^{5}+720 N^{3}\right) t_{1}^{6} t_{2}^{2}}{180 g^{3} M^{5}}+\cdots \\
& \cdots+\frac{\left(105 N^{6}+840 N^{4}\right) t_{1}^{8} t_{2}}{5760 g^{4} M^{5}}+\cdots+\frac{945 N^{5} t_{1}^{10}}{10 ! g^{5} M^{5}}+\cdots
\end{aligned}
$$

Программа для MAPLE, генерирующая разложение интеграла

$$
Z=\frac{\int d \phi \exp \left(-\frac{1}{2} \operatorname{Tr} \phi^{2}+\sum_{k=1} g^{k} t_{k} \operatorname{Tr} \phi^{k}\right)}{\int d \phi \exp \left(-\frac{1}{2} \operatorname{Tr} \phi^{2}\right)}
$$

в рядпо $g$ и $t_{k}$, написанная В. Пестуном, доступна по адресу http://wwwth.itep.ru/progs/ giv.mws или .../giv.tar. Она генерирует первые члены разложения функции $Z$ в ряд и требует использования, по крайней мере, MAPLE 7.

\section{2. НЕГАУССОВЫ СТАТИСТИЧЕСКИЕ СУММЫ}

Из условий Вирасоро в случае негауссова потенциала можно получить негауссовы плотности и полиплотности. Например, простейшая одноточечная плотность для рода нуль имеет вид

$$
\rho_{W}^{(0 \mid 1)}(z)=\frac{W^{\prime}(z)-y(z)}{2}
$$

здесь

$$
y_{W}^{2}(z)=W^{\prime}(z)^{2}-4 f_{W}^{(0 \mid 1)}(z) .
$$

Из уравнения (I.3.40) следуют выражения для всех негауссовых (поли)плотностей, например

$$
\rho_{W}^{(0 \mid 2)}(z, x)=\frac{1}{y(z)}\left(\partial_{x}\left(\frac{\rho_{W}^{(0 \mid 1)}(x)-\rho_{W}^{(0 \mid 1)}(z)}{x-z}\right)+f_{W}^{(0 \mid 2)}(z \mid x)\right)
$$

и

$$
\rho_{W}^{(1 \mid 1)}(z)=\frac{1}{y(z)}\left(\rho_{W}^{(0 \mid 2)}(z \mid z)+f_{W}^{(1 \mid 1)}(z)\right)
$$

Можно показать явно, что функция $\rho_{W}^{(0 \mid 2)}(z, x)$ симметрична по $z$ и $x$, если $f_{W}^{(0 \mid 2)}(z \mid x)$ определена формулой (I.3.39):

$$
f_{W}^{(0 \mid 2)}(z \mid x)=\check{R}_{W}(z) \rho_{W}^{(0 \mid 1)}(x) .
$$


Двухточечная функция может быть представлена в виде, явно симметричном по $x$ и $z$, a именно:

$$
\begin{aligned}
\rho_{W}^{(0 \mid 2)}(z, x)= & \frac{1}{2 y(x) y(z)(x-z)^{2}}\left(W^{\prime}(x) W^{\prime}(z)-y(x) y(z)+\right. \\
& \left.+(x-z)^{2} \check{K}_{W}(x, z) F^{(0)}+\check{A}_{W}(x, z) F^{(0)}\right)
\end{aligned}
$$

где

$$
\check{K}_{W}(x, z)=\sum_{n, m=0} n m T_{n} T_{m} \sum_{i=0}^{n-2} \sum_{j=0}^{m-2} x^{i} z^{j} \frac{\partial}{\partial T_{n-i-2}} \frac{\partial}{\partial T_{m-j-2}}
$$

и

$$
\begin{aligned}
\check{A}_{W}(x, z)= & 2 \sum n T_{n}\left(2 \frac{\partial}{\partial T_{n-2}}+\right. \\
& \left.+\sum_{i=1}^{n-2}\left[(n-i)(x-z)\left(x^{i-1}-z^{i-1}\right)+\left(z x^{i-1}+x z^{i-1}\right)\right] \frac{\partial}{\partial T_{n-i-2}}\right) .
\end{aligned}
$$

Таким образом, двухточечная функция имеет гладкий предел при $x \rightarrow z$ :

$$
\begin{aligned}
& \rho_{W}^{(0 \mid 2)}(x, x)=\frac{1}{2 y(x)^{2}}\left(\frac{1}{8}\left(W^{(3)} W^{\prime}-2 W^{\prime \prime 2}\right)-\right. \\
& -\frac{1}{4 y(x)^{2}}\left(\left(\left(W^{\prime \prime}\right)^{2}+W^{\prime} W^{(3)}-2 \check{R}_{W}^{\prime \prime} F^{(0)}\right)\left(W^{\prime 2}-4 \check{R}_{W}^{\prime} F^{(0)}\right)-\right. \\
& \left.\left.-2\left(W^{\prime} W^{\prime \prime}-2 \check{R}_{W}^{\prime} F^{(0)}\right)^{2}\right)+\check{K}_{W}(x, x) F^{(0)}+\check{B}(x) F^{(0)}\right) \\
& \check{B}(x)=\frac{1}{2} \sum n T_{n} \sum_{i=1}^{n-2}(4(n-i)(i-2)+(i-1)(i-4)) x^{i-2} \frac{\partial}{\partial T_{n-i-2}}, \\
& \langle\langle\operatorname{tr} I\rangle\rangle_{W}^{(0)}=\sum_{i=1}^{n} \widetilde{S}_{i}, \\
& \left\langle\left\langle\operatorname{tr} \phi^{k}\right\rangle\right\rangle_{W}^{(0)}=\sum_{i=1}^{n} \widetilde{S}_{i} \alpha_{i}^{k} \quad \text { для } 0 \leqslant k \leqslant n, \\
& \left\langle\left\langle\operatorname{tr} \phi^{n+1}\right\rangle\right\rangle_{W}^{(0)}=\sum_{i=1}^{n} \widetilde{S}_{i} \alpha_{i}^{n+1}+\frac{1}{(n+1) T_{n+1}}\left(\sum_{i=1}^{n} \widetilde{S}_{i}\right)^{2} .
\end{aligned}
$$

\section{3. ПРЕПОТЕНЦИАЛ КИВ-ДВ: ПРЕДСТАВЛЕНИЕ $\left.\mathcal{F}_{m}^{\mathrm{DV}}\right|_{t=0}$ ЧЕРЕЗ ГАУССОВЫ ПРЕПОТЕНЦИАЛЫ}

Здесь мы из формулы (II.2.4) получим (обобщая результаты работы [23]) первые члены разложения препотенциала $\left.\mathcal{F}_{m}^{\mathrm{DV}}\right|_{t=0}$ по гауссовым препотенциалам. Наши результаты совпадают с результатами теории Зайберга-Виттена (см. [50], [51], [39]):

$$
\left.\mathcal{F}_{1}^{\mathrm{DV}}\right|_{t=0}=-\sum_{i} S_{i} W\left(\alpha_{i}\right)+\left.F_{1}\right|_{t_{k}^{(i)}=-T_{k}^{(i)}}
$$




$$
\begin{aligned}
\left.\mathcal{F}_{2}^{\mathrm{DV}}\right|_{t=0} & =-\frac{1}{2} \sum_{i<j}\left(S_{i}^{2}-4 S_{i} S_{j}+S_{j}^{2}\right) \ln \alpha_{i j}-\frac{3}{4} \sum_{i} S_{i}^{2}+ \\
& +\left[F_{2}-(S \partial) F_{1}+\frac{1}{2} \sum_{k \geqslant 1}^{\infty} \frac{g^{2 k-2}}{k !}(c \partial \partial)^{k} F_{1}^{2}\right]_{t_{k}^{(i)}=-T_{k}^{(i)}} \\
\left.\mathcal{F}_{3}^{\mathrm{DV}}\right|_{t=0} & =\left[F_{3}-(S \partial) F_{2}+\frac{1}{2}(S \partial)^{2} F_{1}+\right. \\
& +\sum_{k \geqslant 1}^{\infty} \frac{g^{2 k-2}}{k !}(c \partial \partial)^{k}\left[F_{1} F_{2}-F_{1}(S \partial) F_{1}\right]+ \\
& \left.+\frac{1}{6} \sum_{k \geqslant 2}^{\infty} \frac{g^{2 k-4}}{k !}\left((c \partial \partial)^{k} F_{1}^{3}-3 F_{1}(c \partial \partial)^{k} F_{1}^{2}\right)\right]_{t_{k}^{(i)}=-T_{k}^{(i)}} \\
\left.\mathcal{F}_{4}^{\mathrm{DV}}\right|_{t=0} & =\left[F_{4}-(S \partial) F_{3}+\frac{1}{2}(S \partial)^{2} F_{2}-\frac{1}{6}(S \partial)^{3} F_{1}+\right. \\
& +\sum_{k \geqslant 1}^{\infty} \frac{g^{2 k-2}}{k !}(c \partial \partial)^{k}\left[\frac{1}{2} F_{2}^{2}+F_{1} F_{3}-F_{2}(S \partial) F_{1}-\right. \\
& \left.-F_{1}(S \partial) F_{2}+\frac{1}{2} F_{1}(S \partial)^{2} F_{1}+\frac{1}{2}\left[(S \partial) F_{1}\right]^{2}\right]+ \\
& +\frac{1}{2} \sum_{k \geqslant 2}^{\infty} \frac{g^{2 k-4}}{k !}\left[(c \partial \partial)^{k} F_{1}^{2} F_{2}-2 F_{1}(c \partial \partial)^{k} F_{1} F_{2}-F_{2}(c \partial \partial)^{k} F_{1}^{2}\right]+ \\
& +\frac{1}{2} \sum_{k \geqslant 2}^{\infty} \frac{g^{2 k-4}}{k !}\left[(c \partial \partial)^{k} F_{1}^{2}(S \partial) F_{1}-2 F_{1}(c \partial \partial)^{k} F_{1}(S \partial) F_{1}-(S \partial) F_{1}(c \partial \partial)^{k} F_{1}^{2}\right]+ \\
& +\sum_{k \geqslant 3}^{\infty} \frac{1}{24} \frac{g^{2 k-6}}{k !}\left[(c \partial \partial)^{k} F_{1}^{4}+12 F_{1}^{2}(c \partial \partial)^{k} F_{1}^{2}-4 F_{1}(c \partial \partial)^{k} F_{1}^{3}-\right. \\
& \left.\left.-3((c \partial \partial) \otimes I+I \otimes(c \partial \partial))^{k} F_{1}^{2} \otimes F_{1}^{2}\right]\right]_{t_{k}^{(i)}=-T_{k}^{(i)}}
\end{aligned}
$$

3.1. Явные формулы для $\left.\mathcal{F}_{\mathrm{DV}}^{(p \mid m)}\right|_{t=0}$. $m=1$ :

$$
\begin{aligned}
\left.\mathcal{F}_{\mathrm{DV}}^{(0 \mid 1)}\right|_{t=0} & =-\sum_{i} S_{i} W\left(\alpha_{i}\right) \\
\left.\mathcal{F}_{\mathrm{DV}}^{(1 \mid 1)}\right|_{t=0} & =\sum_{i} \frac{S_{i}}{M_{i}}\left(-\frac{\left\langle\left\langle 4^{1}\right\rangle\right\rangle^{(1)}}{4} \sigma_{2}^{(i)}+\frac{\left\langle\left\langle 3^{2}\right\rangle\right\rangle^{(1)}}{2 ! \cdot 3^{2}}\left[\sigma_{1}^{(i)}\right]^{2}\right)= \\
& =\sum_{i} \frac{S_{i}}{M_{i}}\left(-\frac{\sigma_{2}^{(i)}}{4}+\frac{3\left[\sigma_{1}^{(i)}\right]^{2}}{2 ! \cdot 3^{2}}\right)=\sum_{i} \frac{S_{i}}{12 M_{i}}\left(2\left[\sigma_{1}^{(i)}\right]^{2}-3 \sigma_{2}^{(i)}\right) \\
\left.\mathcal{F}_{\mathrm{DV}}^{(2 \mid 1)}\right|_{t=0} & =\sum_{i} \frac{S_{i}}{M_{i}^{3}}\left(-\frac{\left\langle\left\langle 8^{1}\right\rangle\right\rangle^{(2)}}{8} \sigma_{6}^{(i)}+\frac{\left\langle\left\langle 3^{1}, 7^{1}\right\rangle\right\rangle^{(2)}}{3 \cdot 7} \sigma_{1}^{(i)} \sigma_{5}^{(i)}+\frac{\left\langle\left\langle 4^{1}, 6^{1}\right\rangle\right\rangle^{(2)}}{4 \cdot 6} \sigma_{2}^{(i)} \sigma_{4}^{(i)}+\right.
\end{aligned}
$$




$$
\begin{aligned}
& +\frac{\left\langle\left\langle 5^{2}\right\rangle\right\rangle^{(2)}}{2 ! \cdot 5^{2}}\left[\sigma_{3}^{(i)}\right]^{2}-\frac{\left.\left\langle 3^{2}, 6^{1}\right\rangle\right\rangle^{(2)}}{2 ! \cdot 3^{2} \cdot 6}\left[\sigma_{1}^{(i)}\right]^{2} \sigma_{4}^{(i)}-\frac{\left\langle\left\langle 3^{1}, 4^{1}, 5^{1}\right\rangle\right\rangle^{(2)}}{3 \cdot 4 \cdot 5} \sigma_{1}^{(i)} \sigma_{2}^{(i)} \sigma_{3}^{(i)}- \\
& -\frac{\left\langle\left\langle 4^{3}\right\rangle\right\rangle^{(2)}}{3 ! \cdot 4^{3}}\left[\sigma_{2}^{(i)}\right]^{3}+\frac{\left\langle\left\langle 3^{2}, 4^{2}\right\rangle\right\rangle^{(2)}}{(2 !)^{2} \cdot 3^{2} \cdot 4^{2}}\left[\sigma_{1}^{(i)}\right]^{2}\left[\sigma_{2}^{(i)}\right]^{2}- \\
& \left.-\frac{\left.\left\langle 3^{4}, 4^{1}\right\rangle\right\rangle^{(2)}}{4 ! \cdot 3^{4} \cdot 4}\left[\sigma_{1}^{(i)}\right]^{4} \sigma_{2}^{(i)}+\frac{\left\langle\left\langle 3^{6}\right\rangle\right\rangle^{(2)}}{6 ! \cdot 3^{6}}\left[\sigma_{1}^{(i)}\right]^{6}\right) .
\end{aligned}
$$

$m=2$ :

$$
\begin{aligned}
\left.\mathcal{F}_{\mathrm{DV}}^{(0 \mid 2)}\right|_{t=0}= & -\frac{1}{2} \sum_{i<j}\left(S_{i}^{2}-4 S_{i} S_{j}+S_{j}^{2}\right) \ln \alpha_{i j}-\frac{3}{4} \sum_{i} S_{i}^{2} \\
\left.\mathcal{F}_{\mathrm{DV}}^{(1 \mid 2)}\right|_{t=0}= & \sum_{i} \frac{S_{i}^{2}}{M_{i}^{2}}\left(-\frac{\left\langle\left\langle 6^{1}\right\rangle\right\rangle^{(1)}}{6} \sigma_{4}^{(i)}+\frac{\left\langle\left\langle 4^{2}\right\rangle\right\rangle^{(1)}}{2 ! \cdot 4^{2}}\left[\sigma_{2}^{(i)}\right]^{2}+\frac{\langle\langle 3,5\rangle\rangle^{(1)}}{3 \cdot 5} \sigma_{1}^{(i)} \sigma_{3}^{(i)}-\right. \\
& \left.-\frac{\left\langle\left\langle 3^{2}, 4\right\rangle\right\rangle^{(1)}}{2 ! \cdot 3^{2} \cdot 4}\left[\sigma_{1}^{(i)}\right]^{2} \sigma_{2}^{(i)}+\frac{\left\langle\left\langle 3^{4}\right\rangle\right\rangle^{(1)}}{4 ! \cdot 3^{4}}\left[\sigma_{1}^{(i)}\right]^{4}\right)- \\
& -\sum_{i \neq j} \frac{S_{i} S_{j}}{M_{i}^{2}}\left(\frac{\left.\left\langle 4^{1}\right\rangle\right\rangle^{(1)}}{2 \alpha_{i j}^{4}}+\frac{2\left\langle\left\langle 3^{2}\right\rangle\right\rangle^{(1)}}{9 \alpha_{i j}^{3}} \sigma_{1}^{(i)}-\frac{\langle\langle 2,4\rangle\rangle^{(1)}}{4 \alpha_{i j}^{2}} \sigma_{2}^{(i)}+\right. \\
& +\frac{\left\langle\left\langle 2^{1}, 3^{2}\right\rangle\right\rangle^{(1)}}{2 ! \cdot 3^{2} \alpha_{i j}^{2}}\left[\sigma_{1}^{(i)}\right]^{2}+\frac{2\langle\langle 1,5\rangle\rangle^{(1)}}{5 \alpha_{i j}} \sigma_{3}^{(i)}+\frac{2\langle\langle 1,3,4\rangle\rangle^{(1)}}{3 \cdot 4 \alpha_{i j}} \sigma_{1}^{(i)} \sigma_{2}^{(i)}+ \\
& \left.+\frac{2\left\langle\left\langle 1,3^{3}\right\rangle\right\rangle^{(1)}}{3 ! \cdot 3^{3} \alpha_{i j}}\left[\sigma_{1}^{(i)}\right]^{3}\right)+\sum_{i \neq j} \frac{S_{i} S_{j}}{M_{i} M_{j} \alpha_{i j}^{4}}\left[\left\langle\left\langle 1^{2}\right\rangle\right\rangle^{(0)}\right]^{2} .
\end{aligned}
$$

$m=3:$

$$
\begin{aligned}
\left.\mathcal{F}_{\mathrm{DV}}^{(0 \mid 3)}\right|_{t=0}= & {\left[F_{3}-(S \partial) F_{2}+\frac{1}{2}(S \partial)^{2} F_{1}+\sum_{k \geqslant 1}^{\infty} \frac{g^{2 k-2}}{k !}(c \partial \partial)^{k}\left[F_{1} F_{2}-F_{1}(S \partial) F_{1}\right]+\right.} \\
& \left.+\frac{1}{6} \sum_{k \geqslant 2}^{\infty} \frac{g^{2 k-4}}{k !}\left((c \partial \partial)^{k} F_{1}^{3}-3 F_{1}(c \partial \partial)^{k} F_{1}^{2}\right)\right]_{t_{k}^{(i)}=-T_{k}^{(i)}}= \\
= & \sum_{i=1}^{n}\left[S_{i}^{3}\left(\frac{2 t_{4}^{(i)}}{M_{i}^{2}}+\frac{6\left[t_{3}^{(i)}\right]^{2}}{M_{i}^{3}}\right)-(S \partial) S_{i}^{2}\left(\frac{t_{2}^{(i)}}{M_{i}}+\frac{3 t_{1}^{(i)} t_{3}^{(i)}}{M_{i}^{2}}\right)+\right. \\
& \left.+\frac{1}{2}(S \partial)^{2} S_{i} \frac{\left[t_{1}^{(i)}\right]^{2}}{2 M_{i}}\right]_{t_{k}^{(i)}=-T_{k}^{(i)}}=\sum_{i}\left(\frac{S_{i}^{3}}{M_{i}}\left(-\frac{2 \sigma_{2}^{(i)}}{4}+\frac{6\left[\sigma_{1}^{(i)}\right]^{2}}{3^{2}}\right)-\right. \\
& \left.-\sum_{j \neq i} \frac{2 S_{i}^{2} S_{j}}{M_{i}}\left(\frac{1}{2 \alpha_{i j}^{2}}+\frac{\sigma_{1}^{(i)}}{\alpha_{i j}}\right)+\sum_{j, k \neq i} \frac{2 S_{i} S_{j} S_{k}}{M_{i} \alpha_{i j} \alpha_{i k}}\right)= \\
= & \frac{2}{3} \sum_{i} \frac{S_{i}^{3}}{M_{i}}\left(\sum_{j \neq i} \frac{1}{\alpha_{i j}^{2}}+\frac{5}{4} \sum_{j<k} \frac{1}{\alpha_{i j} \alpha_{i k}}\right)+ \\
& +\sum_{i \neq j} S_{i}^{2} S_{j}\left(\frac{2}{M_{j} \alpha_{i j}^{2}}-\frac{3}{M_{i} \alpha_{i j}^{2}}-\frac{2}{\alpha_{i j}} \sum_{k \neq i, j} \frac{1}{\alpha_{i k}}\right)+
\end{aligned}
$$




$$
+4 \sum_{i<j<k} S_{i} S_{j} S_{k}\left(\frac{1}{M_{i} \alpha_{i j} \alpha_{i k}}+\frac{1}{M_{j} \alpha_{j i} \alpha_{j k}}+\frac{1}{M_{k} \alpha_{k i} \alpha_{k j}}\right)
$$

Согласно предположению Р. Дийкграафа и К. Вафы [25] это выражение совпадает (после замены $\left.S_{[51]}=2 S, \mathcal{F}_{[51]}=2 \mathcal{F}\right)$ с результатами вычислений [51] в рамках теории Зайберга-Виттена [35]-[39]. Согласно [51]

$$
\begin{aligned}
\left.\mathcal{F}_{\mathrm{DV}}^{(0 \mid 3)}\right|_{t=0} & =\sum_{i} u_{i} S_{i}^{3}+\sum_{i \neq j} u_{i j} S_{i}^{2} S_{j}+\sum_{i<j<k} u_{i j k} S_{i} S_{j} S_{k}, \\
u_{i} & =\frac{1}{6}\left(-\sum_{j \neq i} \frac{1}{M_{j} \alpha_{i j}^{2}}+\frac{1}{4 M_{i}} \sum_{j<k} \frac{1}{\alpha_{i j} \alpha_{i k}}\right)= \\
& =\frac{1}{6 M_{i}}\left(\sum_{j \neq i} \frac{1}{\alpha_{i j}^{2}}+\frac{5}{4} \sum_{\substack{j<k \\
j, k \neq i}} \frac{1}{\alpha_{i j} \alpha_{i k}}\right), \\
u_{i j} & =\frac{1}{4}\left(-\frac{3}{M_{i} \alpha_{i j}^{2}}+\frac{2}{M_{j} \alpha_{i j}^{2}}-\frac{2}{M_{i} \alpha_{i j}} \sum_{k \neq i, j} \frac{1}{\alpha_{i k}}\right), \\
u_{i j k} & =\frac{1}{M_{i} \alpha_{i j} \alpha_{i k}}+\frac{1}{M_{j} \alpha_{j i} \alpha_{j k}}+\frac{1}{M_{k} \alpha_{k i} \alpha_{k j}},
\end{aligned}
$$

и два выражения ${ }^{13)}$ для $u_{i}$ совпадают вследствие правила сумм [39]

$$
\sum_{j \neq i} \frac{1}{\alpha_{i j}^{2} M_{j}}=-\frac{1}{M_{i}} \sum_{j \neq i} \frac{1}{\alpha_{i j}^{2}}-\frac{1}{M_{i}} \sum_{\substack{j<k \\ j, k \neq i}} \frac{1}{\alpha_{i j} \alpha_{i k}}=0,
$$

полученного с помощью суммирования по всем вычетам $\alpha_{i}$ в интеграле

$$
\oint_{\alpha_{i}} \frac{d x}{\left(x-\alpha_{i}\right)^{2} P_{n}(x)}=\oint_{0} \frac{d z}{z^{3} \prod_{j \neq i}\left(\alpha_{i j}+z\right)}=\frac{1}{M_{i}}\left(\sum_{j \neq i} \frac{1}{\alpha_{i j}^{2}}+\sum_{\substack{j<k \\ j, k \neq i}} \frac{1}{\alpha_{i j} \alpha_{i k}}\right) .
$$

3.2. Пример $n=2$ (кубический препотенциал $W(z), 3 T_{3}=\kappa, M_{1}=-M_{2}=$ $\left.\kappa \alpha_{12}\right)$. В случае $n=2$ единственный нетривиальный параметр $\sigma$ удовлетворяет соотношению $\sigma_{1}^{(1)}=-\sigma_{1}^{(2)}=1 / \alpha_{12}$, и, используя приведенные вьше формулы, мы с легкостью восстанавливаем старый результат [23]:

$$
\begin{aligned}
& \left.\mathcal{F}_{\mathrm{DV}}^{(0 \mid 1)}\right|_{t=0}=-S_{1} W\left(\alpha_{1}\right)-S_{2} W\left(\alpha_{2}\right), \\
& \left.\mathcal{F}_{\mathrm{DV}}^{(1 \mid 1)}\right|_{t=0}=\frac{S_{1}-S_{2}}{6 \kappa \alpha_{12}^{3}}, \\
& \left.\mathcal{F}_{\mathrm{DV}}^{(2 \mid 1)}\right|_{t=0}=\frac{\left(S_{1}-S_{2}\right)\left\langle\left\langle 3^{6}\right\rangle\right\rangle(2)}{6 ! \cdot 3^{6} \kappa^{3} \alpha_{12}^{9}}=\frac{35\left(S_{1}-S_{2}\right)}{6 \kappa^{3} \alpha_{12}^{9}},
\end{aligned}
$$

13) Тождественность (III.3.10) и (III.3.11) была впервые продемонстрирована В. Пестуном (январь 2003, неопубликовано). 


$$
\begin{aligned}
\left.\mathcal{F}_{\mathrm{DV}}^{(0 \mid 2)}\right|_{t=0}= & -\frac{1}{2}\left(S_{1}^{2}-4 S_{1} S_{2}+S_{2}^{2}\right) \ln \alpha_{12}, \\
\left.\mathcal{F}_{\mathrm{DV}}^{(1 \mid 2)}\right|_{t=0}= & \frac{\left(S_{1}^{2}+S_{2}^{2}\right)\left\langle\left\langle 3^{4}\right\rangle\right\rangle^{(1)}}{4 ! \cdot 3^{4} \kappa^{2} \alpha_{12}^{6}}- \\
& -\frac{2 S_{1} S_{2}}{\kappa^{2} \alpha_{12}^{6}}\left(\frac{\left\langle\left\langle 4^{1}\right\rangle\right\rangle^{(1)}}{2}+\frac{2\left\langle\left\langle 3^{2}\right\rangle\right\rangle^{(1)}}{9}+\frac{\left\langle\left\langle 2,3^{2}\right\rangle\right\rangle^{(1)}}{2 ! \cdot 3^{2}}+\right. \\
& \left.+\frac{2\left\langle\left\langle 1^{1}, 3^{3}\right\rangle\right\rangle^{(1)}}{3 ! \cdot 3^{3}}+\left[\left\langle\left\langle 1^{2}\right\rangle\right\rangle^{(0)}\right]^{2}\right)= \\
= & \frac{1}{\kappa^{2} \alpha_{12}^{6}}\left(\frac{4536}{8 \cdot 3^{5}}\left(S_{1}^{2}+S_{2}^{2}\right)-\left(1+\frac{4 \cdot 3}{9}+\frac{2 \cdot 18}{18}+\frac{4 \cdot 162}{3^{4}}+2\right) S_{1} S_{2}\right)= \\
= & \frac{1}{\kappa^{2} \alpha_{12}^{6}}\left(\frac{7}{3}\left(S_{1}^{2}+S_{2}^{2}\right)-\frac{31}{3} S_{1} S_{2}\right) \\
\left.\mathcal{F}_{\mathrm{DV}}^{(0 \mid 3)}\right|_{t=0}= & \frac{6}{3^{2}} \frac{S_{1}^{3}-S_{2}^{3}}{\kappa \alpha_{12}^{3}}-\frac{5 S_{1} S_{2}\left(S_{1}-S_{2}\right)}{\kappa \alpha_{12}^{3}}= \\
= & \frac{2}{3 \kappa \alpha_{12}^{3}}\left(S_{1}^{3}-S_{2}^{3}-\frac{15}{2} S_{1} S_{2}\left(S_{1}-S_{2}\right)\right) .
\end{aligned}
$$

\section{IV. КОММЕНТАРИИ}

\section{1. ОБОБЩЕННОЕ ПРАВИЛО СУММ (II.1.25): ВЫВОД И ПРИМЕНЕНИЕ}

Обобшенное правило сумм (II.1.25) может быть выведено многими различными способами (некоторые из которых можно найти, например, в работе [41]). Здесь мы представим еше один возможный вывод. Для простоты мы положим $M$ равным 1. Зависимость от $M$ можно легко восстановить.

Из первого уравнения иерархии цепочки Тоды можно получить рекуррентное соотношение для плотности

$$
\rho_{N+1}(x)+\rho_{N-1}(x)-2 \rho_{N}(x)=\frac{\partial_{x}^{2} \rho_{N}(x)}{N} .
$$

Непосредственно из этого уравнения можно вычислить все плотности с $N>1$, но мы воспользуемся другим подходом. А именно, мы введем производяшую функцию для гауссовых плотностей

$$
I_{\lambda}(x)=\sum_{N=1}^{\infty} \rho_{N}(x) \lambda^{N}
$$

Как легко видеть, из уравнения (IV.1.1) следует дифференциальное уравнение для этой производящей функции. Точнее говоря, мы имеем

$$
2 \operatorname{sh}^{2}(\varphi) \partial_{\varphi} J_{e^{2 \varphi}}(x)=\partial_{x}^{2} J_{e^{2 \varphi}}(x),
$$

где

$$
J_{e^{2 \varphi}}(x)=\operatorname{sh}^{2}(\varphi) I_{e^{2 \varphi}}(x) .
$$


Применяя обратное преобразование Лапласа к функции $J$, получаем

$$
2 \operatorname{sh}^{2}(\varphi) \partial_{\varphi} \hat{J}(p)=p^{2} \hat{J}(p)
$$

где $\hat{J}(p)$ - образ обратного преобразования Лапласа

$$
\hat{J}(x)=\int_{0}^{\infty} e^{-x p} \hat{J}(p) d p
$$

Это дифференциальное уравнение можно решить, получив общее решение вида

$$
\hat{I}_{e^{2 \varphi}}(p)=\frac{\exp \left(-\frac{p^{2} \operatorname{cth}(\varphi)}{2}\right)}{\operatorname{sh}^{2}(\varphi)} f(p)
$$

Для того чтобы найти постоянную интегрирования $f(p)$, можно определить производящую функцию для $N=1$ и сравнить ее с явно вычисленной. Таким образом получим $f(p)=1 / 4$. Чтобы получить обобшенное правило сумм (II.1.25), необходимо рассмотреть образ при преобразовании Лапласа функции $I_{\lambda}$,

$$
I_{\lambda}(p)=\frac{\lambda}{(1-\lambda)^{2}} \exp \left(\frac{p^{2}}{2} \frac{1+\lambda}{1-\lambda}\right)
$$

т.е. интеграл

$$
\frac{\lambda}{(1-\lambda)^{2}} \int_{0}^{\infty} e^{-p x} \exp \left(\frac{p^{2}}{2} \frac{1+\lambda}{1-\lambda}\right),
$$

который расходится. Для того чтобы иметь возможность работать с этим интегралом, необходимо рассматривать его как формальный ряд по $1 / x$. Тогда после простого вычисления получаем

$$
\begin{aligned}
I_{\lambda}(x) & =\frac{1}{\sqrt{2 \pi}} \frac{x \lambda}{(1-\lambda)^{2}} \int \frac{1}{x^{2}-t^{2} \frac{1+\lambda}{1-\lambda}} e^{-t^{2} / 2} d t= \\
& =\frac{1}{\sqrt{2 \pi}} \sum_{N=1}^{\infty} x \int\left(\left[\frac{x^{2}+t^{2}}{x^{2}-t^{2}}\right]^{N}-1\right) e^{-t^{2} / 2} \frac{d t}{2 t^{2}} \lambda^{N}
\end{aligned}
$$

и коэффициенты разложения в ряд по $\lambda$ дают требуемое правило сумм. $\mathrm{K}$ сожалению, мы не знаем обобщения этого или какого-либо другого вывода на случай гауссовых полиплотностей, равно как и на случай негауссовых (поли)плотностей.

Используя правило сумм, легко можно восстановить разложение по родам. Вводя переменную $\tilde{x}$ такую, что $\tilde{x}^{2}=x^{2} / N$, получаем разложение по родам в виде разложения

3 Теоретическая и математическая физика, т. 142, № 3,2005 г. 
по $1 / N^{2}$ :

$$
\begin{aligned}
\rho_{N}(x) & =\frac{x}{\sqrt{2 \pi}} \int\left(\left[\frac{x^{2}+t^{2}}{x^{2}-t^{2}}\right]^{N}-1\right) e^{-t^{2} / 2} \frac{d t}{2 t^{2}}= \\
& =\frac{x}{\sqrt{2 \pi}} \int\left(e^{2 N t^{2} / x^{2}} \exp \left(N \sum_{k=1}^{\infty}\left(\frac{t^{2}}{x^{2}}\right)^{2 k+1} \frac{2}{(2 k+1)}\right)-1\right) e^{-t^{2} / 2} \frac{d t}{2 t^{2}}= \\
& =\frac{x}{\sqrt{2 \pi}} \int\left(e^{2 t^{2} / \tilde{x}^{2}} \exp \left(\sum_{k=1}^{\infty}\left(\frac{t^{2}}{\tilde{x}^{2}}-1\right)^{2 k+1} \frac{2}{(2 k+1) N^{2 k}}\right)\right) e^{-t^{2} / 2} \frac{d t}{2 t^{2}}= \\
& =\frac{x \sqrt{\tilde{x}^{2}-4}}{2 \tilde{x} \sqrt{2 \pi}} \int\left(\exp \left(\sum_{k=1}^{\infty}\left(\frac{t^{2}}{\tilde{x}^{2}-4}\right)^{2 k+1} \frac{2}{(2 k+1) N^{2 k}}\right)-e^{-2 t^{2} /\left(\tilde{x}^{2}-4\right)}\right) e^{-t^{2} / 2} \frac{d t}{t^{2}}
\end{aligned}
$$

Разложение этой формулы в рядпо $1 / N^{2}$ задает разложение по родам, и, таким образом, можно получить все выражения из п. III.1.2.

Используя правило сумм, можно получить выражение для коэффициента при старших степенях полинома $Q$ в формуле (III.1.1). Для рода $k$ имеем

$$
\begin{aligned}
\rho^{(1 \mid k)}= & \frac{N \tilde{x} \sqrt{\tilde{x}^{2}-4}}{\tilde{x}}\left(\frac{(4 k-1) ! !}{(2 k+1)\left(\tilde{x}^{2}-4\right)^{2 k+1}}+\right. \\
& \left.+\frac{(4 k+1) ! !}{\left(\tilde{x}^{2}-4\right)^{2 k+2}} \sum_{i=1}^{k} \frac{1}{(2 i+1)(2 k-2 i+1)}+\cdots\right)= \\
= & \frac{N \tilde{x}}{\tilde{x} y^{6 k-1}}\left(\frac{(4 k-1) ! ! y^{2 k-2}}{(2 k+1)}+y^{2 k-4}(4 k+1) ! ! \sum_{i=1}^{k} \frac{1}{(2 i+1)(2 k-2 i+1)}+\cdots\right)= \\
= & \frac{N \tilde{x}}{\tilde{x} y^{6 k-1}}\left(\frac{(4 k-1) ! ! \tilde{x}^{2 k-2}}{(2 k+1)}+\tilde{x}^{2 k-4}\left[-\frac{4(k-1)(4 k-1) ! !}{(2 k+1)}+\right.\right. \\
& \left.\left.+(4 k+1) ! ! \sum_{i=1}^{k} \frac{1}{(2 i+1)(2 k-2 i+1)}\right]+O\left(\tilde{x}^{2 k-6}\right)\right),
\end{aligned}
$$

где $y^{2}=\tilde{x}^{2}-4$.

\section{2. ВЫЧИСЛЕНИЕ ПОЛИПЛОТНОСТЕЙ В ГАУССОВОЙ МОДЕЛИ}

В приншипе все полиплотности в гауссовой модели можно вывести шаг за шагом из условий Вирасоро, как это сделано для нескольких первых условий в п. III.1.4. Но хотелось бы получить выражения типа (II.1.25), которые объединяют все рода. Здесь мы рассмотрим только двухточечную функцию.

Для двухточечной функции сушествует правило сумм при $N=1$ :

$$
\begin{aligned}
& \oint \oint \rho^{(2)}\left(z_{1}, z_{2}\right) z_{1}^{k_{1}} z_{2}^{k_{2}} d z_{1} d z_{2}= \\
& \quad= \begin{cases}\left(2 k_{1}+2 k_{2}-1\right) ! ! & \text { при } k_{1}, k_{2} \text { нечетных, } \\
\left(2 k_{1}+2 k_{2}-1\right) ! !-\left(2 k_{1}-1\right) ! !-\left(2 k_{2}-1\right) ! ! & \text { при } k_{1}, k_{2} \text { четных }, \\
0 & \text { в остальных случаях. }\end{cases}
\end{aligned}
$$


Условия Вирасоро для гауссова потенциала можно объединить следуюшим образом:

$$
\left(x \widehat{\nabla}_{x}-\partial_{0}\right) Z_{N}=\widehat{\nabla}_{x}^{2} Z_{N} .
$$

Тогда для несвязной двухточечной резольвенты получаем

$$
\rho^{(2)}(x, x)+\rho^{(1)}(x)^{2}=\frac{x^{2}}{\sqrt{2 \pi}} \int\left(\left[\frac{x^{2}+t^{2}}{x^{2}-t^{2}}\right]^{N}-1-2 N \frac{t^{2}}{x^{2}}\right) e^{-t^{2} / 2} \frac{d t}{2 t^{2}} .
$$

Из первого уравнения иерархии цепочки Тоды (Т.2.13) легко вывести следуюшее уравнение, которому должна удовлетворять двухточечная резольвента:

$$
\rho_{N+1}^{(2)}(x, y)+\rho_{N-1}^{(2)}(x, y)-2 \rho_{N}^{(2)}(x, y)=\frac{\left(\partial_{x}+\partial_{y}\right)^{2} \rho_{N}^{(2)}(x, y)}{N}-\frac{\partial_{x}^{2} \rho_{N}^{(1)}(x) \partial_{y}^{2} \rho_{N}^{(1)}(y)}{N^{2}} .
$$

Это уравнение совместно с известными значениями младших плотностей, т.е. они удовлетворяют этому уравнению в лидируюшем порядке по $1 / N$.

Используя вычисления с гармоническим осциллятором из работы [41], находим

$$
\eta_{N}^{(2)}(y, z)=\eta_{N}^{(1)}(y+z)-G_{N}(y, z)
$$

где

$G_{N}(y, z)=\exp \left(\frac{z^{2}+y^{2}}{2}\right)\left[\sum_{n=0}^{N-1} B(n, n ; y) B(n, n ; z)+2 \sum_{n=0}^{N-1} \sum_{s=n+1}^{N-1} B(n, s ; y) B(n, s ; z)\right]$.

Здесь $B(n, s ; x)$ - это некоторая (гипергеометрическая) функция, задаюшая подходящий матричный элемент для гармонического осциллятора:

$$
B(n, s ; x)=\sqrt{n ! s !} \sum_{i=0}^{n} \frac{x^{n+s-2 i}}{i !(n-i) !(s-i) !} .
$$

Используя преобразование Лапласа, можно получить двухточечную резольвенту

$$
\rho^{(2)}(s, t)=\int_{0}^{\infty} \int_{0}^{\infty} e^{-(y s+z t)} \eta^{(2)}(y, z) d y d z .
$$

Используя интегральное представление полиномов Эрмита $H_{n}$,

$$
H_{n}(x)=\frac{(-1)^{n} n !}{2 \pi i} \oint \frac{d z}{z^{n+1}} e^{-z^{2} / 2-z x},
$$

можно представить функции $G_{N}(y, z)$ в виде четырехкратных контурных интегралов:

$$
\begin{aligned}
G_{N}(y, z)= & \exp \left(\frac{y^{2}+z^{2}}{2}\right) \times \\
& \times(\oint d z)^{4} \sum_{s, n=0}^{N-1} \frac{s ! n !}{\left(z_{1} z_{3}\right)^{n+1}\left(z_{2} z_{4}\right)^{s+1}} e^{z_{1} z_{2}+z_{3} z_{4}-\left(z_{1}+z_{2}\right) y-\left(z_{3}+z_{4}\right) z}
\end{aligned}
$$


Для производяшей функции имеем

$$
\begin{aligned}
K(\lambda)= & \exp \left(-\frac{y^{2}+z^{2}}{2}\right) \sum_{N=1}^{\infty} G_{N} \lambda^{N}= \\
= & (\oint d z)^{4} \int_{0}^{\infty} \int_{0}^{\infty} d p_{1} d p_{2} e^{-p_{1}-p_{2}} \frac{1}{p_{1}-z_{1} z_{3}} \frac{1}{p_{2}-z_{2} z_{4}} \times \\
& \times\left[\frac{1}{1-\frac{\lambda p_{1} p_{2}}{z_{1} z_{2} z_{3} z_{4}}}-\frac{1}{1-\frac{\lambda p_{2}}{z_{2} z_{4}}}-\frac{1}{1-\frac{\lambda p_{1}}{z_{1} z_{3}}}+\frac{1}{1-\lambda}\right] e^{z_{1} z_{2}+z_{3} z_{4}-\left(z_{1}+z_{2}\right) y-\left(z_{3}+z_{4}\right) z} .
\end{aligned}
$$

Полная производящая функция двухточечных корреляционных функций имеет вид

$$
\begin{aligned}
\sum_{N=1}^{\infty} \eta_{N}^{(2)} \lambda^{N}= & \frac{\lambda}{(1-\lambda)^{2}} \exp \left(\frac{(y+z)^{2}}{2} \frac{1+\lambda}{1-\lambda}\right)-\exp \left(\frac{y^{2}+z^{2}}{2}\right) K(\lambda)= \\
= & \frac{\lambda}{(1-\lambda)^{2}} \exp \left(\left(y^{2}+z^{2}\right) \frac{1+\lambda}{2(1-\lambda)}\right) \times \\
& \times\left(\sum_{k=0}^{\infty} \frac{1}{k !}\left(\frac{z y}{1-\lambda}\right)^{k} \sum_{m=0}^{k}\left(\frac{\lambda y z}{1-\lambda}\right)^{m} \frac{1}{m !}-\right. \\
& \left.-\sum_{k=0}^{\infty} \frac{1}{k !}\left(\frac{\lambda z y}{1-\lambda}\right)^{k} \sum_{m=0}^{k}\left(\frac{y z}{1-\lambda}\right)^{m} \frac{1}{m !}\right)
\end{aligned}
$$

Легко проверить, что эта формула дает ответ, согласованный с формулой (IV.2.6) для $N$ от 1 до 3. Эта формула гораздо сложнее, чем соответствуюшее выражение (IV.1.8) для одноточечной плотности, и получение из нее разложения по родам для двухточечной функции нетривиально.

\section{3. НЕПРЕРЫВНЫЕ ПРЕДЕЛЫ}

Сушествует бесконечное множество непрерывных пределов, и только очень небольшое их количество было до сих пор хотя бы частично исследовано. Среди них:

наивный предел больших $N$ (планарный, или 'т хоофтовский) [54], [55],

двойной скейлинговый предел [56], [37], [38],

предел Беренштейна-Малдасены-Настазе [57],

предел Дийкграафа-Вафы [25], [49], [47].

В каждом из этих пределов ЭММКР переходит в некоторую другую матричную модель. Поэтому естественно сначала исследовать сами эти матричные модели и лиш потом проводить детальный и обоснованный анализ множества непрерывных пределов. Это послужит темой отдельной публикации.

K тому же отдельные представители этих других семейств матричных моделей тождественно эквивалентны ЭММКР, что позволяет проводить анализ пределов в рамках этих семейств: различные непрерывные пределы связаны с различными обобщениями ЭММКР. Одним из примеров является семейство обобшенных моделей Концевича [14], [6], в котором гауссова модель Концевича совпадает с ЭММКР. 
Благодарности. Мы хотим поблагодарить Василия Пестуна за сотрудничество на начальной стадии этого проекта, Юрия Макеенко за важные комментарии о статусе задачи и Наталью Амбург, Леонида Чехова и Антона Хорошкина за полезные обсуждения.

Наша работа была частично поддержана Федеральной программой министерства России по промьшленности, науке и технологиям (грант № 40.052.1.1.1112), а также грантами INTAS № 00-334 (А.С.А), РФФИ № 03-02-17373 (А.С.А), грантом РФФИ для поддержки молодых ученых (А.С.А), грантом фонда “Династия" и МЦФФМ (А.С.А.), грантом Volkswagen Stiftung (А.Д.М. и А.Ю.М.), грантом INTAS № 00-561 (А.Д.М. и А.Ю.М.), грантом РФФИ № 04-02-16538 (А.Д.М.), грантом поддержки ведущих научных школ № 96-15-96798 (А.Д.М.), грантом РФФИ № 04-02-16880 (А.Ю.М.).

\section{Список литературы}

[1] А. М. Поляков. Калибровочные поля и струны. Черноголовка: ИТФ им. Л. Д. Ландау, 1995; М. Грин, Дж. Швари, Э. Виттен. Теория суперструн. Т. 1, 2. М.: Мир, 1990; А. Ю. Морозов. УФН. 1992. Т. 162. С. 83; J. Polchinski. String Theory. V. 1, 2. Cambridge: Cambridge Univ. Press, 1998; A. B. Мариаков. УФH. 2002. T. 172. C. 977; hep-th/0212114.

[2] A. Gerasimov, S. Khoroshkin, D. Lebedev, A. Mironov, A. Morozov. Int. J. Mod. Phys. A. 1995. V. 10. P. 2589; hep-th $/ 9405011 ;$ А. Д. Миронов, А. Ю. Морозов, Л. Винә. ТМФ. 1994. Т. 100. № 1. С. 119; С. М. Харчев, А. Д. Миронов, А. Ю. Морозов. ТМФ. 1995. Т. 104. № 1. С. 129; А. Д. Миронов. ТМФ. 1998. Т. 114. № 2. С. 163; A. Mironov, A. Morozov. Phys. Lett. B. 2002. V. 524. P. 217.

[3] M. L. Mehta. Random matrices. N. Y.: Academic Press, 1991; E. Brézin, C. Itzykson, G. Parisi, J.-B. Zuber. Commun. Math. Phys. 1978. V. 59. P. 35; D. Bessis. Commun. Math. Phys. 1979. V. 69. P. 147; D. Bessis, C. Itzykson, J.-B. Zuber. Adv. Appl. Math. 1980. V. 1. P. 109; C. Itzykson, J.-B. Zuber. J. Math. Phys. 1980. V. 21. P. 411.

[4] A. Gerasimov, A. Marshakov, A. Mironov, A. Morozov, A. Orlov. Nucl. Phys. B. 1991. V. 357. P. 565.

[5] S. Kharchev, A. Marshakov, A. Mironov, A. Orlov, A. Zabrodin. Nucl. Phys. B. 1991. V. 366. P. 569.

[6] А. Ю. Морозов. УФН. 1994. Т. 167. С. 3; hep-th/9303139; Matrix Models as Integrable Systems. hep-th/9502091.

[7] A. Mironov. Int. J. Mod. Phys. A. 1994. V. 9. Р. 4355; А. Д. Миронов. ЭЧАЯ. 2002. T. 33. C. $1051 ;$ I. K. Kostov. Conformal field theory techniques in random matrix models. hep-th/9907060.

[8] A. Marshakov, A. Mironov, A. Morozov. Phys. Lett. B. 1991. V. 265. P. 99; S. Kharchev, A. Marshakov, A. Mironov, A. Morozov, S. Pakuliak. Nucl. Phys. B. 1993. V. 404. P. 717; А. Д. Миронов, С. З. Пакуляк. ТМФ. 1993. Т. 95. № 2. С. 317.

[9] I. Kostov. Phys. Lett. B. 1992. V. 297. P. 74.

[10] J. Alfaro, I. K. Kostov. Generalized Hirota Equations in Models of 2D Quantum Gravity. hep-th/9604011.

[11] M. Mineev-Weinstein, P. B. Wiegmann, A. Zabrodin. Phys. Rev. Lett. 2000. V. 84. P. 5106; I. K. Kostov, I. Krichever, M. Mineev-Weinstein, P. B. Wiegmann, A. Zabrodin. $\tau$-function for analytic curves. In: Random Matrices and Their Applications. MSRI Publs. V. 40. Eds. P. M. Bleher, A. R. Its. Cambridge: Cambridge Univ. Press, 2001. P. 285; hep-th/0005259; A. Boyarsky, A. Marshakov, O. Ruchayskiy, P. Wiegmann, A. Zabrodin. Phys. Lett. B. 2001. V. 515. P. 483; I. Krichever, A. Marshakov, A. Zabrodin. Integrable structure of the Dirichlet boundary problem in multiply-connected domains. hep-th/0309010.

[12] М. Л. Концевич. Функц. анализ и его прилож. 1991. Т. 25. № 2. С. 50. 
[13] E. Witten. On the Kontsevich model and other models of two-dimensional gravity. In: Proc. of the XXth International Conference on Differential Geometric Methods in Theoretical Physics (New York, 1991). Eds. S. Catto, A. Rocha. River Edge: World Sci. Publishing, 1992. V. 1, 2. P. 176; A. Marshakov, A. Mironov, A. Morozov. Phys. Lett. B. 1992. V. 274. P. 280.

[14] S. Kharchev, A. Marshakov, A. Mironov, A. Morozov, A. Zabrodin. Nucl. Phys. B. 1992. V. 380. P. 181; Phys. Lett. B. 1992. V. 275. P. 311.

[15] S. Kharchev, A. Marshakov, A. Mironov, A. Morozov. Nucl. Phys. B. 1993. V. 397. P. 339.

[16] S. Kharchev, A. Marshakov, A. Mironov, A. Morozov. Mod. Phys. Lett. A. 1993. V. 8. P. 1047.

[17] L. Chekhov, Yu. Makeenko. Phys. Lett. B. 1992. V. 278. P. 271; L. Chekhov. Matrix Models and Geometry of Moduli Spaces. hep-th/9509001; S. Kharchev. Kadomtsev-Petviashvili hierarchy and generalized Kontsevich model. hep-th/9810091.

[18] E. P. Wigner. Ann. Math. 1951. V. 53. P. 36.

[19] F. J. Dyson. J. Math. Phys. 1962. V. 3. P. 140; D. Gross, E. Witten. Phys. Rev. D. 1980. V. 21. P. 446; T. Eguchi, H. Kawai. Phys. Rev. Lett. 1982. V. 48. P. 1063; D. V. Voiculescu, K. J. Dykema, A. Nica. Free Random Variables. CRM Monograph Series. V. 70. Providence, RI: AMS, 1992; P. Di Francesco, P. Ginsparg, J. Zinn-Justin. Phys. Rep. 1995. V. 254. P. 1.

[20] F. David. Nucl. Phys. B. 1985. V. 257. P. 45; V. A. Kazakov, I. K. Kostov, A. A. Migdal. Phys. Lett. B. 1985. V. 157. P. 295.

[21] A. Givental. Semisimple Frobenius structures at higher genus. math.AG/0008067.

[22] J. S. Song, Y. S. Song. J. Math. Phys. 2004. V. 45. P. 4539; hep-th/0103254; A. Alexandrov. J. Math. Phys. 2003. V. 44. P. 5268.

[23] F. David. Phys. Lett. B. 1993. V. 302. P. 403; hep-th/9212106; G. Bonnet, F. David, B. Eynard. J. Phys. A. 2000. V. 33. P. 6739; cond-mat/0003324; A. Klemm, M. Mariño, S. Theisen. JHEP. 2003. V. 0303. P. 051.

[24] И. С. Градитейн, И. М. Рыжик. Таблицы интегралов, сумм, рядов и произведений. М.: Наука, 1971.

[25] R. Dijkgraaf, C. Vafa. Nucl. Phys. B. 2002. V. 644. P. 3; P. 21; hep-th/0208048.

[26] A. A. Migdal. Phys. Rep. 1983. V. 102. P. 199; J. Ambjørn, J. Jurkiewicz, Yu. Makeenko. Phys. Lett. B. 1990. V. 251. P. 517.

[27] E. Witten. Nucl. Phys. B. 1996. V. 460. P. 335.

[28] I. A. Batalin, G. A. Vilkovisky. Phys. Lett. B. 1981. V. 102. P. 27; 1983. V. 120. P. 166; Phys. Rev. D. 1983. V. 28. P. 2567; Nucl. Phys. B. 1984. V. 234. P. 106; J. Math. Phys. 1985. V. 26. P. 172.

[29] M. Henneaux, C. Teitelboim. Quantization of Gauge Systems. Princeton: Princeton Univ. Press, 1992; J. Gomis, J. París, S. Samuel. Phys. Rep. 1995. V. 259. P. 1.

[30] E. S. Fradkin, G. A. Vilkovisky. Phys. Lett. B. 1975. V. 55. P. 224; I. A. Batalin, G. A. Vilkovisky. Phys. Lett. B. 1977. V. 69. P. 309.

[31] M. Henneaux. Phys. Rep. 1985. V. 126. P. 1.

[32] E. Witten. Mod. Phys. Lett. A. 1990. V. 5. P. 487.

[33] A. S. Schwarz. Commun. Math. Phys. 1993. V. 155. P. 249.

[34] J. Polchinski. Nucl. Phys. B. 1984. V. 231. P. 269; A. Mironov, A. Morozov. Phys. Lett. B. 2000. V. 490. P. 173.

[35] N. Seiberg, E. Witten. Nucl. Phys. B. 1994. V. 426. P. 19.

[36] F. David. Mod. Phys. Lett. A. 1990. V. 5. P. 1019; A. Mironov, A. Morozov. Phys. Lett. B. 1990. V. 252. P. 47; J. Ambjørn, Yu. Makeenko. Mod. Phys. Lett. A. 1990. V. 5. P. 1753; H. Itoyama, Y. Matsuo. Phys. Lett. B. 1991. V. 255. P. 202.

[37] M. Fukuma, H. Kawai, R. Nakayama. Int. J. Mod. Phys. A. 1991. V. 6. P. 1385; R. Dijkgraaf, H. Verlinde, E. Verlinde. Nucl. Phys. B. 1991. V. 348. P. 435.

[38] Yu. Makeenko, A. Marshakov, A. Mironov, A. Morozov. Nucl. Phys. B. 1991. V. 356. P. 574.

[39] H. Itoyama, A. Morozov. Int. J. Mod. Phys. A. 2003. V. 18. P. 5889. 
[40] J. Harer, D. Zagier. Invent. Math. 1986. V. 85. P. 457; S. K. Lando, A. K. Zvonkin. Embedded graphs. Preprint № 63. Bonn: Max-Plank-Institut für Mathematik, 2001.

[41] C. Itzykson, J.-B. Zuber. Commun. Math. Phys. 1990. V. 134. P. 197.

[42] E. Witten. Nucl. Phys. B. 1990. V. 340. P. 281; R. Dijkgraaf, H. Verlinde, E. Verlinde. Nucl. Phys. B. 1991. V. 352. P. 59; B. Dubrovin. Geometry of 2D topological field theories. In: Integrable Systems and Quantum Groups. Lecture Notes in Math. V. 1620. Eds. M. Francaviglia, S. Greco. Berlin: Springer, 1996. P. 120.

[43] A. Marshakov, A. Mironov, A. Morozov. Phys. Lett. B. 1996. V. 389. P. 43; Mod. Phys. Lett. A. 1997. V. 12. P. 773; Int. J. Mod. Phys. A. 2000. V. 15. P. 1157.

[44] А. С. Лосев. Письма в ЖЭТФ. 1997. Т. 65. С. 374; K. Ito, S.-K. Yang. Phys. Lett. В. 1998. V. 433. P. 56; G. Bertoldi, M. Matone. Phys. Rev. D. 1998. V. 57. P. 6483; A. Morozov. Phys. Lett. B. 1998. V. 427. P. 93; A. Mironov, A. Morozov. Phys. Lett. B. 1998. V. 424. P. 48; H. W. Braden, A. Marshakov, A. Mironov, A. Morozov. Phys. Lett. B. 1999. V. 448. P. 195; A. Veselov. Phys. Lett. A. 1999. V. 261. P. 297; J. M. Isidro. Nucl. Phys. B. 1999. V. 539. P. 379; A. Mironov. WDVV equations and Seiberg-Witten theory. hep-th/9903088; A. В. Мариаков. ТМФ. 2002. Т. 132. С. 3.

[45] F. Cachazo, K. Intriligator, C. Vafa. Nucl. Phys. B. 2001. V. 603. P. 3; F. Cachazo, C. Vafa. $N=1$ and $N=2$ geometry from fluxes. hep-th/0206017.

[46] N. Dorey, T. J. Hollowood, S. Prem Kumar, A. Sinkovics. JHEP. 2002. V. 0211. P. 039; P. 040; V. 0212. P. 003; F. Ferrara. Nucl. Phys. B. 2003. V. 648. P. 161; Phys. Rev. D. 2003. V. 67. P. 085013; D. Berenstein. Phys. Lett. B. 2003. V. 552. P. 255; R. Dijkgraaf, S. Gukov, V. Kazakov, C. Vafa. Phys. Rev. D. 2003. V. 68. P. 045007; A. Gorsky. Phys. Lett. B. 2003. V. 554. P. 185; R. Dijkgraaf, M. T. Grisaru, C. S. Lam, C. Vafa, D. Zanon. Phys. Lett. B. 2003. V. 573. P. 138; B. Feng. Seiberg duality in matrix model. hep-th/0211202; B. Feng. Nucl. Phys. B. 2003. V. 661. P. 113; F. Cachazo, M. R. Douglas, N. Seiberg, E. Witten. JHEP. 2002. V. 0212. P. 071; F. Cachazo, N. Seiberg, E. Witten. JHEP. 2003. V. 0302. P. 042; V. 0304. P. 018; A. Dymarsky, V. Pestun. Phys. Rev. D. 2003. V. 67. P. 125001; R. Boels, J. de Boer, R. Duivenvoorden, J. Wijnhout. JHEP. 2004. V. 0403. P. 010; hep-th/0305189.

[47] G. Bonelli. Nucl. Phys. B. 2003. V. 649. P. 130; hep-th/0209225; H. Fuji, Y. Ookouchi. JHEP. 2002. V. 0212. P. 067; hep-th/0210148; R. Argurio, V. L. Campos, G. Ferretti, R. Heise. Phys. Rev. D. 2003. V. 67. P. 065005; hep-th/0210291; Phys. Lett. B. 2003. V. 553. P. 332; hep-th/0211249; J. McGreevy. JHEP. 2003. V. 0301. P. 047; hep-th $/ 0211009$; H. Suzuki. JHEP. 2003. V. 0303. P. 005; hep-th/0211052; P. 036; hep-th/0212121; I. Bena, R. Roiban. Phys. Lett. B. 2003. V. 555. P. 117; hep-th/0211075; Y. Demasure, R. A. Janik. Phys. Lett. B. 2003. V. 553. P. 105; hep-th/0211082; R. Gopakumar. JHEP. 2003. V. 0305. P. 033; hep-th/0211100; I. Bena, R. Roiban, R. Tatar. Nucl. Phys. B. 2004. V. 679 . P. 168; hep-th/0211271; Y. Tachikawa. Phys. Lett. B. 2003. V. 573. P. 235; hep-th/0211189; Progr. Theor. Phys. 2003. V. 110. P. 841; hep-th/0211274; Y. Ookouchi. JHEP. 2004. V. 0401. P. 014; hep-th/0211287; S. K. Ashok, R. Corrado, N. Halmagyi, K. D. Kennaway, C. Romelsberger. Phys. Rev. D. 2003. V. 67. P. 086004; hep-th/0211291; K. Ohta. JHEP. 2003. V. 0302. P. 057; hep-th/0212025; R. A. Janik, N. A. Obers. Phys. Lett. B. 2003. V. 553. P. 309; hep-th/0212069; S. Seki. Nucl. Phys. B. 2003. V. 661. P. 257; hep-th/0212079; C. Hofman. JHEP. 2003. V. 0310. P. 022; hep-th/0212095; C. H. Ahn, S. Nam. Phys. Lett. B. 2003. V. 562. P. 141; hep-th/0212231; C. H. Ahn. Phys. Lett. B. 2003. V. 560. P. 116; hep-th/0301011; S. Aoyama, T. Masuda. JHEP. 2004. V. 0403. P. 072; hep-th/0309232.

[48] A. Gorsky, I. Krichever, A. Marshakov, A. Mironov, A. Morozov. Phys. Lett. B. 1995. V. 355. P. 466; E. Martinec, N. Warner. Nucl. Phys. B. 1996. V. 459. P. 97; R. Donagi, E. Witten. Nucl. Phys. B. 1996. V. 460. P. 299; A. Gorsky, A. Mironov, A. Marshakov, A. Morozov. Nucl. Phys. B. 1998. V. 527. P. 690; H. Itoyama, A. Morozov. Nucl. Phys. B. 1996. V. 477. P. 855; 1997. V. 491. P. 529; Integrability and Seiberg-Witten theory. hep-th/9601168; E. D'Hoker, D. H. Phong. Lectures on supersymmetric Yang-Mills 
theory and integrable systems. hep-th/9912271; A. Marshakov. Seiberg-Witten Theory and Integrable Systems. Singapore: World Scientific, 1999; H. W. Braden, I. M. Krichever (eds.). Integrability: The Seiberg-Witten and Whitham Equations. Amsterdam: Gordon and Breach, 2000; A. Gorsky, A. Mironov. Integrable many-body systems and gauge theories. hep-th/0011197.

[49] L. Chekhov, A. Mironov. Phys. Lett. B. 2003. V. 552. P. 293; V. Kazakov, A. Marshakov. J. Phys. A. 2003. V. 36. P. 3107.

[50] H. Itoyama, A. Morozov. Nucl. Phys. B. 2003. V. 657. P. 53; Phys. Lett. B. 2003. V. 555. P. 287.

[51] H. Itoyama, A. Morozov. Progr. Theor. Phys. 2003. V. 109. P. 433.

[52] L. Chekhov, A. Marshakov, A. Mironov, D. Vasiliev. Phys. Lett. B. 2003. V. 562. P. 323; A. Mironov. Fortsch. Phys. 2003. V. 51. P. 781.

[53] J. Ambjørn, L. Chekhov, C. F. Kristjansen, Yu. Makeenko. Nucl. Phys. B. 1993. V. 404. P. 127; J. Ambjørn, L. Chekhov, Yu. Makeenko. Phys. Lett. B. 1992. V. 282. P. 341; G. Akemann. Nucl. Phys. B. 1996. V. 482. P. 403

[54] G. 't Hooft. Nucl. Phys. B. 1974. V. 72. P. 461.

[55] G. Veneziano. Nucl. Phys. B. 1976. V. 117. P. 519; D. De Wit, G. 't Hooft. Phys. Lett. 1977. V. 69. P. $61 ;$ E. Witten. The $1 / N$ expansion in atomic and particle physics. In: Recent Developments in Gauge Theories. Proc. of ASI (Cargese/Corsica, France, Aug. $26-$ Sept. 7, 1979). NATO ASI Ser., Ser. B, Physics, V. 59. Eds. G. 't Hooft et al. N. Y.: Plenum, 1980. P. 403; S. R. Wadia. Phys. Rev. D. 1981. V. 24. P. 970; A. Mironov, A. Morozov, G. Semenoff. Int. J. Mod. Phys. A. 1996. V. 11. P. 5031; B. Eynard. JHEP. 2003. V. 0311. P. 018; hep-th/0309036.

[56] E. Brézin, V. A. Kazakov. Phys. Lett. B. 1990. V. 236. P. 144; D. Gross, A. A. Migdal. Phys. Rev. Lett. 1990. V. 64. P. 127; Nucl. Phys. B. 1990. V. 340. P. 333; M. Douglas, S. Shenker. Nucl. Phys. B. 1990. V. 335. P. 635.

[57] D. Berenstein, J. Maldacena, H. Nastase. JHEP. 2002. V. 0204. P. 013; N. R. Constable, D. Z. Freedman, M. Headrick, S. Minwalla. JHEP. 2002. V. 0210. P. 068; D. J. Gross, A. Mikhailov, R. Roiban. Ann. Phys. 2002. V. 301. P. 31; hep-th/0205066; JHEP. 2003. V. 0305. P. 025; N. Beisert, C. Kristjansen, J. Plefka, G. W. Semenoff, M. Staudacher. Nucl. Phys. B. 2002. V. 643. P. 3; 2003. V. 650. P. 125.

Поступила в редакцию $16 . \mathrm{IV} .2004$ г. 